\title{
Critérios Abstratos de Comparação e Aplicações*
}

\author{
Monica Regina Gaiotto
}

Orientador: Prof. Dr. Alexandre Nolasco de Carvalho

Dissertação apresentada ao Instituto de Ciências Matemáticas e de Computação - ICMC-USP, como parte dos requisitos para obtenção do título de Mestre em Ciências - Área: Matemática.

\section{USP - São Carlos}

Maio de 2000 
Louvem a Deus no seu templo,

louvem a ele no seu poderoso firmamento!

Louvem a Deus por suas façanhas,

louvem a ele por sua imensa grandeza!

Louvem a Deus tocando trombetas, louvem a ele com cítara e harpa!

Louvem a Deus com dança e tambor, louvem a ele com cordas e flauta!

Louvem a Deus com címbalos sonoros, louvem a ele com címbalos vibrantes!

Todo o ser que respira louve a Javé!

Salmo 150 
Aos meus pais

Dorival e Nadir 


\section{Agradecimentos $^{1}$}

Agradeço acima de tudo a Deus que esteve e está presente em todos os momentos de minha vida.

Agradeço ao Alexandre pela dedicação, disponibilidade e sobretudo pela paciência em me orientar, aos amigos do ICMC-USP, especialmente à Maria Alice pelo apoio constante, ao Fernando pelo incentivo e ao Sadao pela ajuda em dúvidas de digitação, aos professores do departamento que de alguma forma tive contato e aos professores da UNESP-Rio Claro, principalmente à Rosa e à Nativi pela orientação e apoio nos meus estudos.

Agradeço ainda aos meus pais pelas oportunidades de estudo que me proporcionaram e aos meus irmãos Charles e Robson pelo apoio e incentivo que me deram.

Agradeço também a escola "E.E.P.S.G. Pedro Raphael da Rocha," onde os meus estudos tiveram início.

\footnotetext{
${ }^{1}$ Este trabalho teve suporte financeiro da FAPESP
} 


\section{Abstract}

In this work, we develop abstract comparison methods for semilinear differential equations in Banach spaces and apply them to heat equations and systems of ordinary differential equations. 


\section{Resumo}

Neste trabalho desenvolvemos métodos abstratos de comparação para equações diferenciais semilineares em espaços de Banach e aplicamos estes resultados à equação do calor e aos sistemas de equações diferenciais ordinárias. 


\section{Índice}

1 Preliminares 3

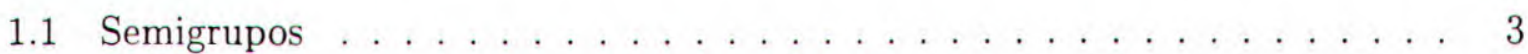

1.2 O Teorema de Hille-Yosida e o Teorema de Lumer-Phillips . . . . . . . . . 5

1.3 Transformada Inversa de Laplace . . . . . . . . . . . . . . . . . . 10

1.4 Operadores Setoriais e Analiticidade . . . . . . . . . . . . . . 12

1.5 Potências Fracionárias . . . . . . . . . . . . . . . . . . . . 15

1.6 Problema de Cauchy não homogêneo . . . . . . . . . . . . . . . . 30

1.7 O Problema de Cauchy Semilinear - Caso Hiperbólico . . . . . . . . . . . . 34

1.8 O Problema de Cauchy Semilinear - Caso Parabólico . . . . . . . . . . . . 39

1.9 O Espaço de Sobolev $W^{1, p} \ldots \ldots \ldots$. . . . . . . . . . . 47

1.10 Existência e Unicidade de Solução Para Problemas de Contorno . . . . . . 50

2 Positividade e Critérios de Comparação Abstratos $\quad 55$

2.1 Espaços de Banach Ordenados e Positividade . . . . . . . . . . . . . 55

2.2 A Equação Linear . . . . . . . . . . . . . . . . . . . . . . . . . . . . . . 59

2.3 Alguns Operadores com Resolvente Positivo . . . . . . . . . . . . . . . 63

2.4 A Equação Não Linear . . . . . . . . . . . . . . . . . . . . . . . . 65

2.4 .1 O caso hiperbólico . . . . . . . . . . . . . . . . 66

2.4 .2 O caso parabólico . . . . . . . . . . . . . . . . . 69

2.5 Sub-soluções e Super-soluções . . . . . . . . . . . . . . . . . . . . 70

3 Aplicações $\quad 73$

3.1 A equação do calor semilinear . . . . . . . . . . . . . . 73

3.2 Um exemplo em dimensão finita . . . . . . . . . . . . . . . . . . 77 


\section{Introdução}

Este trabalho tem por objetivo o estudo de métodos de comparação abstratos para equações diferenciais semilineares em espaços de Banach, afim de encontrar condições suficientes para que soluções de tais problemas possam ser comparadas.

Para um espaço de Hilbert $H$ e um operador $A: D(A) \subset H \rightarrow H$ tal que as seguintes hipóteses estão satisfeitas:

1) $A$ é um operador auto-adjunto e positivo;

2) $(A+\alpha)^{-1} H \subset H$;

3) Para cada $u \in H$ podemos definir $|u| \in H \cap C$ e esta relação satisfaz: Um elemento $u \in H$ está em $C$ se e somente se $u=|u|$ e $\|u\|=\||u|\|$;

4) $\langle|u|, v\rangle \geq|\langle u, v\rangle|, \quad \forall u \in H, \forall v \in C$, mostramos que:

Se $u \in D\left(A^{\frac{1}{2}}\right)$ implica $|u| \in D\left(A^{\frac{1}{2}}\right)$ e

$$
\left\langle A^{\frac{1}{2}}|u|, A^{\frac{1}{2}}|u|\right\rangle \leq\left\langle A^{\frac{1}{2}} u, A^{\frac{1}{2}} u\right\rangle .
$$

então $(A+\alpha)^{-1}$ é crescente para todo $\alpha>0$.

Tal método foi aplicado ao seguinte problema de valor inicial e de fronteira:

$$
\begin{aligned}
& u_{t}(t, x)=u_{x x}(t, x)-u(t, x)+f_{i}(u)(t, x), \quad 0<x<1, t>0 \\
& u(0, x)=u(1, x)=0, \\
& u(0, x)=u_{i}(x) \in H_{0}^{1}, i=0,1 .
\end{aligned}
$$

onde $f_{i}: \mathbb{R} \rightarrow \mathbb{R}, i=0,1$ é diferenciável com derivada localmente limitada. 
Ele foi aplicado também ao sistema de equação diferencial ordinária:

$$
\left\{\begin{array}{l}
\frac{d x}{d t}(t)=A x(t)+f_{i}(x) \\
x(0)=x_{i} \geq 0
\end{array}\right.
$$

onde $A$ é a matriz $n \times n$ dada por:

$$
\left(\begin{array}{ccccc}
1 & -1 & 0 & \cdots & 0 \\
-1 & 2 & -1 & \ddots & \vdots \\
0 & -1 & 2 & \ddots & 0 \\
\vdots & \ddots & \ddots & \ddots & -1 \\
0 & \cdots & 0 & -1 & 1
\end{array}\right)
$$

Com isso, concluímos que se os valores iniciais podem ser comparados, as soluções correspondentes também o são. O mesmo resultado ocorre quando as funções $f_{i}^{\prime} s$ podem ser comparadas.

Para obter os resultados descritos acima, este trabalho foi organizado da seguinte forma:

Capítulo 1: Neste capítulo apresentamos os resultados da teoria de semigrupos necessários ao desenvolvimento do problema proposto. Mostramos também a existência e a unicidade de solução para problemas de contorno em espaços de Sobolev.

Capítulo 2: Desenvolvemos métodos de comparação em espaços de Banach para aplicarmos nos problemas propostos.

Capítulo 3: Para concluir fizemos aplicações dos métodos de comparação à equação do calor e à sistemas de equações diferenciais ordinárias. 


\section{Capítulo 1}

\section{Preliminares}

\section{$1.1 \quad$ Semigrupos}

Este capítulo tem por objetivo apresentar os resultados da teoria de semigrupos necessários ao desenvolvimento do problema proposto. A exposição feita abaixo tem como referência $[8,7,1]$.

Consideraremos $E_{0}$ sempre como um espaço de Banach sobre um corpo $\mathbb{K}$ ( $\mathbb{K}=\mathbb{R}$ ou $\mathbb{C})$, a menos que se diga o contrário. $\mathrm{E}$ denotaremos por $L\left(E_{0}, F_{0}\right)$ o espaço dos operadores $T: E_{0} \rightarrow F_{0}$ lineares e contínuos, munido da norma usual; isto é, para $T \in L\left(E_{0}, F_{0}\right)$,

$$
\|T\|_{L\left(E_{0}, F_{0}\right)}=\sup _{\substack{e \in E_{0} \\ e \neq 0}} \frac{\|T e\|_{F_{0}}}{\|e\|_{E_{0}}} .
$$

Seja $E_{0}^{*}$ o dual topológico de $E_{0}$; isto é, $E_{0}^{*}=L\left(E_{0}, \mathbb{K}\right)$, com a norma usual $\|\cdot\|_{E_{0}^{*}}$ $\left(\left\|e^{*}\right\|_{E_{0}^{*}}=\sup \left\{\operatorname{Re}\left\langle e^{*}, e\right\rangle:\|e\|_{E_{0}} \leq 1\right\}\right)$, onde se $e^{*} \in E_{0}^{*}$ e $e \in E_{0}$, denotamos por $\left\langle e^{*}, e\right\rangle$ o valor de $e^{*}$ em $e$. Denotaremos $L\left(E_{0}, E_{0}\right)$ apenas por $L\left(E_{0}\right)$.

Definição 1.1.1. Um semigrupo de operadores lineares em $E_{0}$ é uma familia $\{T(t)$ : $t \geq 0\} \subset L\left(E_{0}\right)$ satisfazendo

(i) $T(0)=I_{E_{0}}$, 
(ii) $T(t+s)=T(t) T(s)$, para todo $t, s \geq 0$.

Um semigrupo é uniformemente contínuo se

$$
\lim _{t \rightarrow 0^{+}}\left\|T(t)-I_{E_{0}}\right\|_{L\left(E_{0}\right)}=0 .
$$

E ainda dizemos que o semigrupo é fortemente contínuo se para todo $e \in E_{0}$, temos:

$$
\lim _{t \rightarrow 0^{+}}\|T(t) e-e\|_{E_{0}}=0 .
$$

Neste caso chamaremos o semigrupo de $C_{0}$-semigrupo.

Definição 1.1.2. Se $\{T(t), t \geq 0\} \subset L\left(E_{0}\right)$ é um $C_{0}$-semigrupo, seu gerador infinitesimal é o operador definido por $A: D(A) \subset E_{0} \rightarrow E_{0}$, onde

$$
D(A)=\left\{e \in E_{0}: \lim _{t \rightarrow 0^{+}} \frac{T(t) e-e}{t} \text { existe }\right\}, \quad A e=\lim _{t \rightarrow 0^{+}} \frac{T(t) e-e}{t} .
$$

$\mathrm{O}$ teorema a seguir mostra que todo semigrupo fortemente contínuo possui uma limitação exponencial.

Teorema 1.1.1. Suponha que $\{T(t), t \geq 0\} \subset L\left(E_{0}\right)$ é um $C_{0}$ - semigrupo então existem $M \geq 1$ e $\beta \in \mathbb{R}$ tais que

$$
\|T(t)\|_{L\left(E_{0}\right)} \leq M \mathrm{e}^{\beta t}, \quad \forall t \geq 0 .
$$

Quando podemos tomar $M=1$ e $\beta=0$ no teorema anterior, isto é, se

$$
\|T(t)\| \leq 1, \forall t \geq 0
$$

dizemos que $T(t)$ é um $C_{0}$-semigrupo de contrações.

$O$ resultado a seguir dá uma caracterização dos semigrupos uniformemente contínuos de operadores através de seus geradores.

Teorema 1.1.2. Dado um $C_{0}$-semigrupo $\{T(t), t \geq 0\} \subset L\left(E_{0}\right)$, as seguintes afirmativas são equivalentes:

(i) O semigrupo é uniformemente contínuo,

(ii) $O$ seu gerador infinitesimal está definido em todo $E_{0}$,

(iii Para algum $A$ em $L\left(E_{0}\right), T(t)=\mathrm{e}^{t A}$. 
O teorema abaixo estabelece alguns resultados importantes da teoria de semigrupos fortemente contínuos.

Antes de enunciarmos tal teorema, precisamos lembrar que o conjunto resolvente $\rho(A)$ de um operador linear $A$ é o conjunto dos números complexos $\lambda$ tais que $\lambda I-A$ é invertível. Chamaremos invertível uma aplicação que é injetora, sua imagem é densa no contradomínio e sua inversa é limitada. O complementar de $\rho(A)$ em $\mathbb{C}$ é denominado espectro de $A$ e é denotado por $\sigma(A)$.

Teorema 1.1.3. Seja $\{T(t), t \geq 0\} \subset L\left(E_{0}\right)$ um $C_{0}$-semigrupo e $A$ o seu gerador infinitesimal.

1. A é densamente definido e fechado. Para e $\in D(A), t \mapsto T(t) e$ é continuamente diferenciável e

$$
\frac{d}{d t} T(t) e=A T(t) e=T(t) A e, \quad t>0
$$

2. $\cap_{m \geq 1} D\left(A^{m}\right)$ é denso em $E_{0}$.

3. Para $\operatorname{Re} \lambda>\beta$ e $\beta$ dado no Teorema 1.1.1, $\lambda$ está no resolvente $\rho(A)$ de $A$ e

$$
(\lambda-A)^{-1} e=\int_{0}^{\infty} \mathrm{e}^{-\lambda t} T(t) e d t, \quad \forall e \in E_{0} .
$$

Teorema 1.1.4. Sejam $\{T(t), t \geq 0\}$ e $\{S(t), t \geq 0\}$ semigrupos fortemente contínuos cujos geradores são respectivamente $A$ e $B$. Se $A=B$ então $T(t)=S(t), t \geq 0$.

\subsection{O Teorema de Hille-Yosida e o Teorema de Lumer- Phillips}

Os teoremas desta seçāo caracterizam os geradores de semigrupos fortemente contínuos de operadores lineares.

Teorema 1.2.1 (Hille-Yosida). Suponha que $A: D(A) \subset E_{0} \rightarrow E_{0}$ é um operador linear. Então os fatos seguintes são equivalentes: 
(i) A é o gerador infinitesimal de um semigrupo fortemente contínuo $\{T(t), t \geq 0\} \subset$ $L\left(E_{0}\right)$ tal que

$$
\|T(t)\|_{L\left(E_{0}\right)} \leq \mathrm{e}^{\omega t}, \quad \forall t \geq 0
$$

(ii) A é um operador linear fechado, densamente definido cujo conjunto resolvente contém $(\omega, \infty) e$

$$
\left\|(\lambda-A)^{-1}\right\|_{L\left(E_{0}\right)} \leq \frac{1}{\lambda-\omega}, \quad \forall \lambda>\omega
$$

Ambas as condições (i) e (ii) dependem da escolha da norma em $E_{0}$. Daremos uma formulação independente da norma, mas na prática devemos usualmente procurar normas especiais para a qual este teorema se aplica.

Lema 1.2.1. Suponha que A é um operador linear cujo conjunto resolvente contém $(0, \infty)$ e que satisfaz

$$
\left\|(\lambda-A)^{-n}\right\|_{L\left(E_{0}\right)} \leq M \lambda^{-n}, \quad n \geq 1, \lambda>0 .
$$

Então existe uma norma $|\cdot|_{E_{0}}$ em $E_{0}$ tal que

$$
\|e\|_{E_{0}} \leq|e|_{E_{0}} \leq M\|e\|_{E_{0}}, \quad \forall e \in E_{0}
$$

$e$

$$
\left|(\lambda-A)^{-1} e\right|_{E_{0}} \leq \lambda^{-1}|e|_{E_{0}}, \quad \forall e \in E_{0}, \lambda>0
$$

Teorema 1.2.2 (Forma Geral do Teorema de Hille-Yosida). Suponha que A : $D(A) \subset E_{0} \rightarrow E_{0}$ seja um operador linear. As seguintes afirmativas são equivalentes:

(i) A é o gerador de um $C_{0}$-semigrupo $\{T(t), t \geq 0\} \subset L\left(E_{0}\right)$ tal que

$$
\|T(t)\|_{L\left(E_{0}\right)} \leq M \mathrm{e}^{\beta t}, \quad \forall t \geq 0
$$

(ii) A é fechado, $\overline{D(A)}=E_{0}, \rho(A) \supset(\beta, \infty) e$

$$
\left\|(\lambda-A)^{-n}\right\|_{L\left(E_{0}\right)} \leq M(\lambda-\beta)^{-n}, \quad \forall \lambda>\beta, n=1,2, \cdots
$$


Definição 1.2.1. Um operador linear $A: D(A) \subset E_{0} \rightarrow E_{0}$ é dissipativo se para cada $e \in D(A\rangle$ existe $e^{*} \in J(e)$-tal que $\operatorname{Re}\left(e^{*}, A e\right\rangle \leq 0$, onde $J: E_{0} \rightarrow 2^{E_{0}^{*}}$ é uma função multívoca, chamada aplicação dualidade, definida por

$$
J(e)=\left\{e^{*} \in E_{0}^{*}: \operatorname{Re}\left\langle e^{*}, e\right\rangle=\|e\|_{E_{0}}^{2},\left\|e^{*}\right\|_{E_{0}^{*}}=\|e\|_{E_{0}}\right\} .
$$

$J(e) \neq \varnothing$, pelo Teorema de Hahn-Banach.

Lema 1.2.2. Suponha que $A$ é um operador linear. A é dissipativo se e somente se

$$
\|(\lambda-A) e\|_{E_{0}} \geq \lambda\|e\|_{E_{0}}
$$

para todo $e \in D(A)$ e $\lambda>0$.

Teorema 1.2.3 (Lumer-Phillips). Seja $A$ um operador linear sobre $E_{0}$ tal que $\overline{D(A)}=$ $E_{0}$.

(i) Se $A$ é o gerador de um $C_{0}$-semigrupo de contraçôes, então $A$ é dissipativo (ou podemos concluir mais ainda, $\operatorname{Re}\left\langle e^{*}, A e\right\rangle \leq 0$ para todo $\left.e^{*} \in J(e)\right)$ e $R(\lambda-A)=E_{0}$ para todo $\lambda>0$,

(ii) Se A é dissipativo e existe um $\lambda_{0}>0$ tal que $R\left(\lambda_{0}-A\right)=E_{0}$, então $A$ é o gerador de um $C_{0}$-semigrupo de contrą̧óes.

Note que se $A: D(A) \subset E_{0} \rightarrow E_{0}$ é um operador linear tal que $\overline{D(A)}=E_{0}$, o adjunto de $A$ é o operador

$$
A^{*}: D\left(A^{*}\right) \subset E_{0}^{*} \rightarrow E_{0}^{*}
$$

dado por: $D\left(A^{*}\right)$ é o conjunto dos $e^{*} \in E_{0}^{*}$ tais que existe $f^{*} \in E_{0}^{*}$ com

$$
\left\langle e^{*}, A e\right\rangle=\left\langle f^{*}, e\right\rangle, \forall e \in D(A\rangle
$$

Para $e^{*} \in D\left(A^{*}\right)$ escrevemos $A^{*} e^{*}=f^{*}$.

E ainda se $E_{0}$ é um espaço de Banach complexo e $A$ é um operador linear. Chamamos de imagem numérica $W(A)$ do operador A o conjunto

$$
W(A):=\left\{\left\langle e^{*} . A e\right\rangle: e \in D(A), e^{*} \in E_{0}^{*},\|e\|_{E_{0}}=\left\|e^{*}\right\|_{E_{0}^{*}}=1,\left\langle e^{*}, e\right\rangle=1\right\} .
$$


Teorema 1.2.4. Suponha que $A: D(A) \subset E_{0} \rightarrow E_{0}$ é um operador linear fechado tal que $\overline{D(A)}=E_{0}$. Seja $W(A)$ a imagem numérica de $A$ e $\Sigma$ um subconjunto aberto e conexo em $\mathbb{C} \backslash W(A)$. Se $\lambda \notin \overline{W(A)}$ então $\lambda-A$ é injetor, tem imagem fechada e satisfaz

$$
\|(\lambda-A) e\|_{L\left(E_{0}\right)} \geq d(\lambda, W(A))\|e\|_{E_{0}}
$$

Além disso, se $\rho(A) \cap \Sigma \neq \varnothing$ então $\rho(A) \supset \Sigma e$

$$
\left\|(\lambda-A)^{-1}\right\|_{L\left(E_{0}\right)} \leq \frac{1}{d(\lambda, W(A))}, \quad \forall \lambda \in \Sigma
$$

onde $d(\lambda, W(A))$ é a distância de $\lambda$ a $W(A)$.

Definição 1.2.2. Seja $H$ um espaço de Hilbert com produto interno $(\cdot, \cdot)$. Um operador $A: D(A) \subset H \rightarrow H$ é simétrico se $\overline{D(A)}=H$ e $A \subset A^{*} ;$ isto é, $\langle A e, f\rangle=\langle e, A f\rangle$ para todo $e, f \in D(A)$. A é auto-adjunto se $A=A^{*}$.

Exemplo 1.2.1. Seja $H$ um espaço de Hilbert e $A: D(A) \subset H \rightarrow H$ um operador autoadjunto. Segue que $A$ é fechado e $\overline{D(A)}=H$. Suponha que A seja limitado superiormente; isto é, que exista uma constante $a \in \mathbb{R}$ tal que $\langle A u, u\rangle \leq a\langle u, u\rangle$. Então $\mathbb{C} \backslash(-\infty, a] \subset$ $\rho(A)$, e existe uma constante $M \geq 1$ dependendo somente de $\varphi$ tal que

$$
\left\|(A-\lambda)^{-1}\right\|_{L\left(E_{0}\right)} \leq \frac{M}{|\lambda-a|}
$$

para todo $\lambda \in \Sigma_{a}=\{\lambda \in \mathbb{C}: \arg (\lambda-a) \leq \varphi\}, \varphi<\pi$. Segue que $A$ é o gerador de um $C_{0}$-semigrupo $\{T(t): t \geq 0\}$ satisfazendo

$$
\|T(t)\|_{L(H)} \leq \mathrm{e}^{a t}
$$

$N a$ verdade $\{T(t): t \geq 0\}$ é um semigrupo analítico como mostraremos posteriormente.

Teorema 1.2.5. Seja $\{T(t): t \geq 0\}$ um $C_{0}$-semigrupo em $E_{0}$. Suponha que

$$
A(h) e=\frac{T(h) e-e}{h}
$$

então para todo $e \in E_{0}$ temos

$$
T(t) e=\lim _{h \rightarrow 0^{+}} \mathrm{e}^{t A(h)} e
$$

e o limite é uniforme em $t$ em qualquer intervalo limitado de $\mathbb{R}$. 
Teorema 1.2.6. Seja $A$ o gerador de um $C_{0}$-semigrupo $\{T(t): t \geq 0\}$ em $E_{0}$. Então

$$
T(t) e=\lim _{n \rightarrow \infty}\left(I-\frac{t}{n} A\right)^{-n} e=\lim _{n \rightarrow \infty}\left[\frac{n}{t}\left(\frac{n}{t}-A\right)^{-1}\right]^{n} e, \quad \forall e \in E_{0}
$$

e os limites são uniformes para $t$ em intervalos limitados de $\mathbb{R}$.

\subsection{Transformada Inversa de Laplace}

Sabemos que o resolvente do gerador de um $C_{0}$-semigrupo é a transformada de Laplace do semigrupo, pois no Teorema 1.1.3, (3), vimos que

$$
(\lambda-A)^{-1}=\int_{0}^{\infty} \mathrm{e}^{-\lambda t} T(t) d t,
$$

se $\operatorname{Re} \lambda$ é grande. Isto sugere a transformada inversa de Laplace para encontrar $T(t)$, dado $A$. No que se segue perseguiremos este objetivo.

\section{Lema 1.3.1.}

(i) $\int_{-\infty}^{\infty} \frac{\sin t}{t} d t=\pi$

(ii) Se $f: \mathbb{R} \rightarrow \mathbb{C}$ é tal que $f(t) /(1+|t|)$ é integrável em $\mathbb{R} e \int_{-1}^{1}\left|\frac{f(t)-f(0)}{t}\right| d t<\infty$, então

$$
\int_{-\infty}^{\infty} f(t) \frac{\sin N t}{\pi t} d t \rightarrow f(0) \quad \text { quando } N \rightarrow+\infty
$$

Teorema 1.3.1. Seja $A$ o gerador de um $C_{0}$-semigrupo $\{T(t), t \geq 0\} \subset L\left(E_{0}\right)$ satisfazendo $\|T(t)\|_{L\left(E_{0}\right)} \leq M \mathrm{e}^{\beta t}$ e assuma que $\gamma>\max \{0, \beta\}$. Temos que para qualquer e $\in D\left(A^{2}\right)$ et $>0$

$$
T(t) e=\lim _{N \rightarrow \infty} \frac{1}{2 \pi i} \int_{\gamma-i N}^{\gamma+i N} \mathrm{e}^{\lambda t}(\lambda-A)^{-1} e d \lambda,
$$

onde a integral é ao longo do segmento de reta com $\operatorname{Re} \lambda=\gamma$. O limite é uniforme em intervalos da forma $\epsilon \leq t \leq 1 / \epsilon$, qualquer $\epsilon>0$. 
Prova: Peln Teorema 1.1.3, (3) e pelo Teorema de Hille-Yosida, temos que para $\operatorname{Re} \lambda=$ $\gamma>\beta,(\lambda-A)^{-1}$ existe e é uniformemente limitada ( pois $\left\|(\lambda-A)^{-1}\right\| \leq \frac{M}{(\gamma-\beta)}$ ). Se $e \in D\left(A^{2}\right)$

$$
(\lambda-A)^{-1} e=\lambda^{-1} e+\lambda^{-2} A e+\lambda^{-2}(\lambda-A)^{-1} A^{2} e
$$

então

$$
\frac{1}{2 \pi i} \int_{\gamma-i N}^{\gamma+i N} \mathrm{e}^{\lambda t}(\lambda-A)^{-1} e d \lambda=\left(\frac{1}{2 \pi i} \int_{\gamma-i N}^{\gamma+i N} \frac{\mathbf{e}^{\lambda t}}{\lambda} d \lambda\right) e+\frac{1}{2 \pi i} \int_{\gamma-i N}^{\gamma+i N} \frac{\mathrm{e}^{\lambda t}}{\lambda^{2}}\left[A e+(\lambda-A)^{-1} A^{2} e\right] d \lambda
$$

Mas,

$$
\begin{gathered}
\left\|\mathrm{e}^{\lambda t}\left[A e+(\lambda-A)^{-1} A^{2} e\right]\right\| \leq\left|\mathrm{e}^{\lambda t}\right|\left(\|A e\|+\left\|(\lambda-A)^{-1}\right\|\left\|A^{2} e\right\|\right) \leq \\
\leq \mathrm{e}^{\gamma t}\left(\|A e\|+\frac{M}{\gamma-\beta}\left\|A^{2} e\right\|\right) \leq \mathrm{e}^{\frac{\gamma}{\epsilon}}\left(\|A e\|+\frac{M}{\gamma-\beta}\left\|A^{2} e\right\|\right)=\underline{c t e}
\end{gathered}
$$

$$
\left|\int_{\gamma-i N}^{\gamma+i N} \frac{\mathrm{e}^{\lambda t}}{\lambda} d \lambda\right|=\left|\int_{-N}^{N} \frac{\mathrm{e}^{(\gamma+i \theta) t}}{(\gamma+i \theta)} d \theta\right| \leq \int_{-N}^{N}\left|\frac{\mathrm{e}^{(\gamma+i \theta) t}}{t(\gamma+i \theta)^{2}}\right||d \theta| \leq \epsilon^{-1} \int_{-N}^{N} \frac{\mathrm{e}^{\frac{\gamma}{\epsilon}}}{\left|(\gamma+i \theta)^{2}\right|}|d \theta|
$$

Com isso, concluímos que ambos os termos convergem uniformemente em $\epsilon \leq t \leq 1 / \epsilon$ quando $N \rightarrow \infty$, o primeiro por integração por partes e o segundo porque o integrando tem norma menor ou igual a cte $/|\lambda|^{2}$ então converge absolutamente, conforme foi visto acima. Só resta mostrar que o limite é $T(t) e$.

Agora para $\operatorname{Re} \lambda=\gamma$

$$
(\lambda-A)^{-1} e=\int_{0}^{\infty} \mathrm{e}^{-\lambda s} T(s) e d s,
$$

então

$$
\begin{aligned}
\frac{1}{2 \pi i} \int_{\gamma-i N}^{\gamma+i N} \mathrm{e}^{\lambda t}(\lambda-A)^{-1} e d \lambda & =\int_{0}^{\infty}\left\{\frac{1}{2 \pi i} \int_{\gamma-i N}^{\gamma+i N} \mathrm{e}^{\lambda(t-s)} d \lambda\right\} T(s) e d s \\
& =\int_{0}^{\infty} \frac{\sin N(t-s)}{\pi(t-s)} \mathrm{e}^{\gamma(t-s)} T(s) e d s \\
& =\int_{-t}^{\infty} \frac{\sin N \tau}{\pi \tau} \mathbf{e}^{-\gamma \tau} T(t+\tau) e d \tau .
\end{aligned}
$$

Consideremos a seguinte função

$$
f(\tau)=\left\{\begin{array}{l}
\left\langle e^{*}, T(t+\tau) e\right) \mathrm{e}^{-\gamma \tau}, \quad \tau \geq-t \\
0, \quad \tau<-t
\end{array}\right.
$$


Verifiquemos que $f$ satisfaz as condições do lema para qualquer $e^{*} \in E_{0}^{*}$ e $t>0$. Através da definição de diferenciabildade, é fácil mostrar que $f$ é diferenciável em $\tau=0$ e que $f^{\prime}(0)=\left\langle e^{*}, T(t)(A-\gamma) e\right\rangle$. Como $f$ é diferenciável em $[-1,1]$, então pelo teorema do valor médio $\exists c \in(-1,1)$ tal que

$$
\int_{-1}^{1} \frac{|f(\tau)-f(0)|}{|\tau|} d \tau=\int_{-1}^{1} f^{\prime}(c) d \tau=2 f^{\prime}(c)<\infty .
$$

E para concluir as condiçōes do lema temos usando a linearidade de $e^{*}$ e a limitação exponencial que $\frac{f(\tau)}{1+|\tau|}$ é integrável em $\mathbb{R}$.

Logo temos que

$$
\begin{gathered}
\left\langle e^{*}, \frac{1}{2 \pi i} \int_{\gamma-i N}^{\gamma+i N} \mathrm{e}^{\lambda t}(\lambda-A)^{-1} e d \lambda\right\rangle=\left\langle e^{*}, \int_{-t}^{\infty} \frac{\sin N \tau}{\pi \tau} \mathbf{e}^{-\gamma \tau} T(t+\tau) e d \tau\right\rangle= \\
=\int_{-t}^{\infty} \frac{\sin N \tau}{\pi \tau}\left\langle e^{*}, T(t+\tau) e\right\rangle \mathrm{e}^{-\gamma \tau} d \tau \rightarrow f(0)=\left\langle e^{*}, T(t) e\right\rangle
\end{gathered}
$$

quando $N \rightarrow \infty$ pelo Lema, parte ii).

Como a convergência acima vale para todo $e^{*} \in E_{0}^{*}$, então temos que:

$$
\frac{1}{2 \pi i} \int_{\gamma-i N}^{\gamma+i N} \mathrm{e}^{\lambda t}(\lambda-A)^{-1} e d \lambda \rightarrow T(t) e
$$

na topologia fraca, quando $N \rightarrow \infty$. Mas provamos no início que tal integral converge fortemente e como o limite fraco é único, segue que

$$
\frac{1}{2 \pi i} \int_{\gamma-i N}^{\gamma+i N} \mathrm{e}^{\lambda t}(\lambda-A)^{-1} e d \lambda \rightarrow T(t) e
$$

uniformemente para $\epsilon \leq t \leq 1 / \epsilon$, qualquer $\epsilon>0$.

\subsection{Operadores Setoriais e Analiticidade}

Se $A$ é o gerador infinitesimal de um $C_{0}$-semigrupo tal que

$$
\Sigma=\{\lambda \in \mathbb{C}:|\arg \lambda|<\phi\} \subset \rho(A)
$$

para algum $\phi \in(\pi / 2, \pi) \mathrm{e}$

$$
\left\|(\lambda-A)^{-1}\right\|_{L\left(E_{0}\right)} \leq \frac{C}{|\lambda|}, \quad \lambda \in \Sigma
$$

dizemos que $-A$ é setorial. 
Definiçāo 1.4.1. Seja $\Delta=\left\{z \in \mathbb{C}: \phi_{1}<\arg (z)<\phi_{2}, \phi_{1}<0<\phi_{2}\right\}$ e para $z \in \Delta$ seja $T(z)$ um operador linear limitado. A familia $T(z) ; z \in \Delta$ é um semigrupo analítico em $\Delta s e$

(i) $z \mapsto T(z)$ é analítica em $\Delta$,

(ii) $T(0)=I$ e $T(z) e \rightarrow e$ quando $z \rightarrow 0, z \in \Delta$, para todo $e \in E_{0}$,

(iii) $T\left(z_{1}+z_{2}\right)=T\left(z_{1}\right) T\left(z_{2}\right), z_{1}, z_{2} \in \Delta$.

Um semigrupo $T(t)$ é analítico se é analítico em algum setor $\Delta, \operatorname{com}[0, \infty) \subset \Delta$.

Teorema 1.4.1. Seja $A$ um operador tal que $\overline{D(A)}=E_{0} e-A$ é setorial. Então o semigrupo gerado por $A$ é analítico.

Teorema 1.4.2. Suponha que $A: D(A) \subset E_{0} \rightarrow E_{0}$ seja um operador tal que $\overrightarrow{D(A)}=$ $E_{0} e-A$ é setorial; isto é, existem constantes $a, C$ e $\varphi \in(\pi / 2, \pi], \Sigma_{a}=\{\lambda \in \mathbb{C}$ : $|\arg (\lambda-a)|<\varphi\}$ está no conjunto resolvente de $A e$

$$
\left\|(\lambda-A)^{-1}\right\|_{L\left(E_{0}\right)} \leq C /|\lambda-a| \quad \text { em } \Sigma_{a}
$$

Então A é o gerador infinitesimal de um $C_{0}$-semigrupo $\{T(t), t \geq 0\} \subset L\left(E_{0}\right)$,

$$
T(t)=\frac{1}{2 \pi i} \int_{\Gamma_{a}} \mathrm{e}^{\lambda t}(\lambda-A)^{-1} d \lambda
$$

onde $\Gamma_{a}$ é a fronteira de $\Sigma_{a} \backslash\{\lambda \in \mathbb{C}:|\lambda-a| \leq r\}$,r pequeno, orientada com o sentido da parte imaginária crescente. Para algum $K>0$

$$
\|T(t)\|_{L\left(E_{0}\right)} \leq K \mathrm{e}^{a t}, \quad\|A T(t)\|_{L\left(E_{0}\right)} \leq K t^{-1} \mathrm{e}^{a t}
$$

para todo $t>0$. Temos também que

$$
\frac{d}{d t} T(t)=A T(t)
$$

é um operador limitado para qualquer $t>0$. 
Prova: Notemos que basta fazer o caso $a=0$, pois se $A$ é o gerador de

$$
T(t)=\frac{1}{2 \pi i} \int_{\Gamma_{a}} \mathrm{e}^{\lambda t}(\lambda-A)^{-1} d \lambda
$$

então tome $\lambda=a+\mu$, temos

$$
\mathrm{e}^{-a t} T(t)=\frac{1}{2 \pi i} \int_{\Gamma_{a}} \mathrm{e}^{(\lambda-a) t}(\lambda-A)^{-1} d \lambda=\frac{1}{2 \pi i} \int_{\Gamma_{0}} \mathrm{e}^{\mu t}(\mu-(A-a))^{-1} d \mu
$$

e $\left\|(\lambda-A)^{-1}\right\|_{L\left(E_{0}\right)} \leq C /|\lambda-a|$ implica que $\|\left(\mu-(A-a)^{-1} \|_{L\left(E_{0}\right)} \leq C /|\mu|\right.$.

Provaremos inicialmente que $\|T(t)\|_{L\left(E_{0}\right)}$ e $t \| A\left(T(t) \|_{L\left(E_{0}\right)}\right.$ são limitados para $t>0$. Fazendo uma mudança de variáveis $\mu=\lambda t$ temos:

$$
T(t)=\frac{1}{2 \pi i} \int_{\Gamma_{0}} \mathrm{e}^{\mu}\left(\frac{\mu}{t}-A\right)^{-1} \frac{d \mu}{t} .
$$

Logo

$$
\|T(t)\|_{L\left(E_{0}\right)} \leq \frac{1}{2 \pi} \int_{\Gamma_{0}} \mathrm{e}^{\mathrm{Re} \mu}\left\|\left(\frac{\mu}{t}-A\right)^{-1}\right\|_{L\left(E_{0}\right)} \frac{|d \mu|}{t} \leq \frac{1}{2 \pi} \int_{\Gamma_{0}} \mathrm{e}^{\mathrm{Re} \mu} \frac{C}{|\mu| / t} \frac{|d \mu|}{t}=K<\infty
$$

uniformemente para $t>0$. Da mesma forma, temos que:

$$
\begin{aligned}
\frac{1}{2 \pi i} \int_{\Gamma_{0}} \mathrm{e}^{\lambda t} A(\lambda-A)^{-1} d \lambda & =\frac{1}{2 \pi i} \int_{\Gamma_{0}} \mathrm{e}^{\lambda t}\left[-I+\lambda(\lambda-A)^{-1}\right] d \lambda \\
& =-\frac{1}{2 \pi i} \int_{\Gamma_{0}} \mathrm{e}^{\lambda t} d \lambda+\frac{t^{-1}}{2 \pi i} \int_{\Gamma_{0}} \mathrm{e}^{\mu} \frac{\mu}{t}\left(\frac{\mu}{t}-A\right)^{-1} d \mu
\end{aligned}
$$

o primeiro termo é zero e o segundo é estimado da seguinte forma

$$
\left\|\frac{t^{-1}}{2 \pi i} \int_{\Gamma_{0}} \mathrm{e}^{\mu} \frac{\mu}{t}\left(\frac{\mu}{t}-A\right)^{-1} d \mu\right\|_{L\left(E_{0}\right)} \leq \frac{1}{2 \pi t} \int_{\Gamma_{0}} \mathrm{e}^{\mathrm{Re} \mu} C|d \mu|=K_{1} t^{-1}<\infty .
$$

Logo a integral converge. Mostraremos agora que

$$
\frac{1}{2 \pi i} \int_{\Gamma_{0}} \mathrm{e}^{\lambda t} A(\lambda-A)^{-1} d \lambda=A T(t)
$$

Para isso, observe que $A$ é um operador fechado, pois $(\lambda-A)^{-1} \in L\left(E_{0}\right)$ para $\lambda \in \Sigma_{0}$.

Como a integral que define $T(t)$ é um limite de somas de Riemann, isto é,

$$
T(t) e=\lim _{\max \left|\Delta \lambda_{k}\right| \rightarrow 0} \sum_{k} \frac{1}{2 \pi i} \mathrm{e}^{\lambda_{k} t}\left(\lambda_{k}-A\right)^{-1} e \Delta \lambda_{k},
$$

$\lambda_{k} \in \Gamma_{0}$, segue que

$$
A T(t) e=\lim _{\max \left|\Delta \lambda_{k}\right| \rightarrow 0} \Sigma_{k} \frac{1}{2 \pi i} \mathrm{e}^{\lambda_{k} t} A\left(\lambda_{k}-A\right)^{-1} e \Delta \lambda_{k}=\frac{1}{2 \pi i} \int_{\Gamma_{0}} \mathrm{e}^{\lambda t} A(\lambda-A)^{-1} e d \lambda .
$$


Temos também que $A T(t) e=T(t) A e$ para todo $e \in D(A)$.

Pela analiticidade e convergência uniforme para cada $t>0$,

$$
\frac{d}{d t} T(t)=\frac{1}{2 \pi i} \int_{\Gamma_{0}} \mathrm{e}^{\lambda t} \lambda(\lambda-A)^{-1} d \lambda
$$

que é $A T(t)$ como mostrado acima. Com isso, concluímos que:

$$
\|A T(t)\|_{L\left(E_{0}\right)} \leq k_{1} t^{-1}
$$

Seja $e \in D(A), t>0 \mathrm{e}$

$$
T(t) e=\left(\frac{1}{2 \pi i} \int_{\Gamma_{0}} \mathrm{e}^{\lambda t} \frac{d \lambda}{\lambda}\right) e+\frac{t}{2 \pi i} \int_{\Gamma_{0}} \mathrm{e}^{\mu} \frac{\mu}{t}\left(\frac{\mu}{t}-A\right)^{-1} A e \frac{d \mu}{\mu^{2}}
$$

$\log 0$

$$
\|T(t) e-e\|_{E_{0}} \leq \frac{t}{2 \pi} \int_{\Gamma_{0}} \mathrm{e}^{\mathrm{Re} \mu} C\|A e\|_{E_{0}}\left|\frac{d \mu}{\mu^{2}}\right|=\mathcal{O}(t)
$$

quando $t \rightarrow 0^{+}$. Como $\|T(t)\|_{L\left(E_{0}\right)}$ é limitado quando $t \rightarrow 0^{+}, T(t) e \rightarrow e$ quando $t \rightarrow 0^{+}$ para todo $e \in E_{0}$. Finalmente, para $0 \leq s \leq t$ a aplicação $s \mapsto T(t-s) T(s) e$ é contínua e é diferenciável (analítica) para $0<s<t$, com

$$
\frac{d}{d s}(T(t-s) T(s) e)=-A T(t-s) T(s) e+T(t-s) A T(s) e=0
$$

Então $T(t-s) T(s) e=c: \underline{c t e}, \forall e \in E_{0}$ e $0 \leq s \leq t$; em particular

$$
T(t-s) T(s) e=T(t-0) T(0) e,
$$

ou seja,

$$
T(t-s) T(s) e=T(t) e, 0 \leq s \leq t, \forall e \in E_{0} .
$$

Esta é justamente a propriedade de semigrupo: $T(t-s) T(s)=T(t-s+s)=T(t)$. E portanto, a prova de que $T(t)$ é um semigrupo fortemente contínuo está completa. Para completar a prora do teorema, devemos mostrar que $A$ é seu gerador. Mas $T(t) e-e=$ $\int_{0}^{t} T(s) A e d s$, quando $t>0, e \in D(A)$, então $\frac{1}{t}(T(t) e-e) \rightarrow A e$ quando $t \rightarrow 0^{+}$e $A$ está contido no gerador. $A$ é de fato o gerador pois $I$ está no resolvente de $A$ e do gerador. $\square$ 


\subsection{Potências Fracionárias}

Lema 1.5.1. (Série de Neumann) Seja $T \in L(X)$ com $\|T\|<1$, então a série $\sum_{n=0}^{\infty} T^{n}$ é absolutamente convergente $e$

$$
(I-T)^{-1}=\sum_{n=0}^{\infty} T^{n}
$$

Além disso,

$$
\left\|(I-T)^{-1}\right\| \leq(1-\|T\|)^{-1}
$$

Definição 1.5.1. Seja $E$ um espaço de Banach. Um operador linear $A$ em $E$ é dito de tipo positivo com constante $K$ (veja [1]), $K \geq 1$ se é fechado, $\overline{D(A)}=E, \mathbb{R}^{+} \subset$ $\rho(-A) e$

$$
(1+s)\left\|(s+A)^{-1}\right\|_{L(E)} \leq K, \quad s \in \mathbb{R}^{+}
$$

Pelo restante desta seção assumimos que $A$ é um operador de tipo positivo com constante $K$ e denotaremos $A \in P_{K}(E)$.

Dado $s \in \mathbb{R}^{+}$e $\lambda \in \mathbb{C}$ satisfazendo

$$
|\lambda-s| \leq(1+s) /(2 K)
$$

segue de $\lambda+A=(s+A)\left(1+(\lambda-s)(s+A)^{-1}\right)$ que $\lambda \in \rho(-A)$, pois os operadores $(s+A)$ e $\left(1+(\lambda-s)(s+A)^{-1}\right)$ possuem inversa limitada. O primeiro se deve ao fato de que $s \in \rho(-A)$ e o segundo usa-se Série de Neumann. E ainda temos que:

$$
\begin{aligned}
\left\|(\lambda+A)^{-1}\right\|_{L(E)} & \leq\left\|\left[1+(\lambda-s)(s+A)^{-1}\right]^{-1}\right\|_{L(E)}\left\|(s+A)^{-1}\right\|_{L(E)} \leq 2 K(1+s)^{-1} \\
& \leq \frac{2 K}{1+|\lambda|} \frac{1+s+|\lambda-s|}{1+s} \leq \frac{2 K}{1+|\lambda|}\left(1+\frac{1}{2 K}\right)=\frac{2 K+1}{1+|\lambda|} .
\end{aligned}
$$

Observando a Figura 1.1, concluímos que

$$
\sin (\theta)=\frac{s /(2 k)}{s}=\frac{1}{2 k}
$$

e

$$
\Sigma_{K}:=\{z \in \mathbb{C}:|\arg z| \leq \arcsin 1 /(2 K)\}+\{z \in \mathbb{C}:|z| \leq 1 /(2 K)\} \subset \rho(-A)
$$




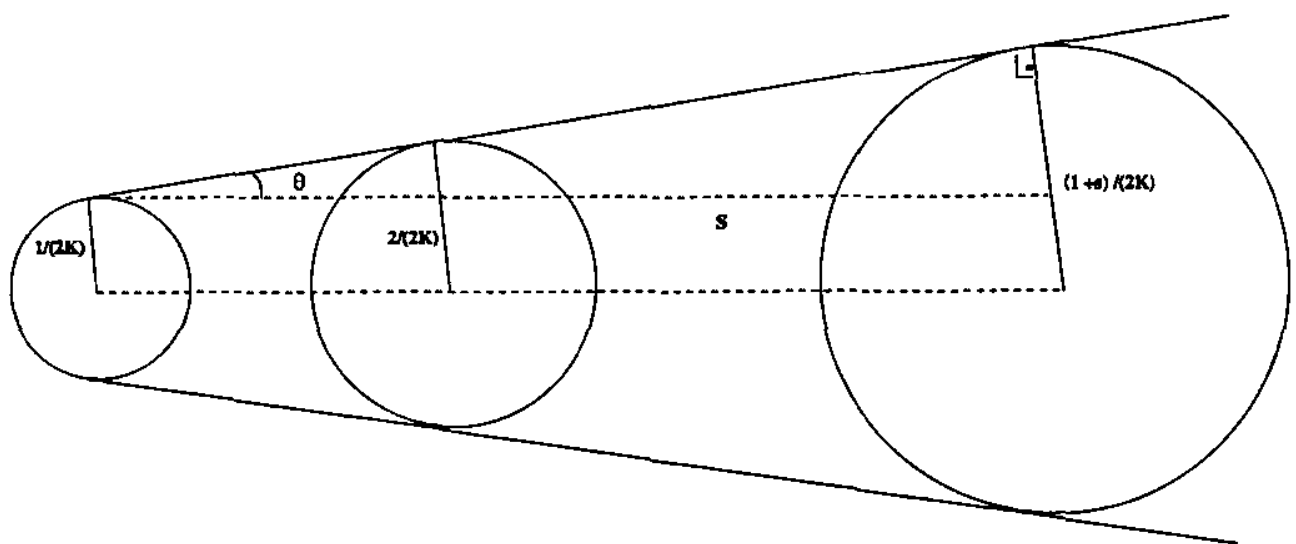

Figura 1.1:

Temos também que:

$$
(1+|\lambda|)\left\|(\lambda+A)^{-1}\right\|_{L(E)} \leq 2 K+1, \quad \lambda \in \Sigma_{K} .
$$

Seja $\Phi$ o conjunto das funções holomorfas $\varphi: \mathbb{C} \backslash\left(-\mathbb{R}^{+}\right) \rightarrow \mathbb{C}$ tal que existe $\delta>0$ (dependendo de $\varphi$ ) com

$$
|\lambda|^{\delta} \varphi(\lambda) \rightarrow 0 \text { quando } \quad|\lambda| \rightarrow \infty,
$$

uniformemente em $\{\lambda \in \mathbb{C}:|\arg \lambda| \leq \pi-\epsilon\}$ para cada $\epsilon \in(0, \pi)$. Temos que $\Phi$ é uma álgebra comutativa sem unidade relativamente a multiplicação ponto a ponto, pois se $\exists \dot{\psi} \in \Phi$ tal que $\psi(\lambda) \varphi(\lambda)=\varphi(\lambda) \quad \forall \varphi \in \Phi e \forall \lambda \in \mathbb{C} \backslash\left(-\mathbb{R}^{+}\right)$, teríamos que $\psi(\lambda)=1=1+0 i$, e entāo $\forall \delta>0$

$$
\lim _{|\lambda| \rightarrow \infty}|\lambda|^{\delta} \psi(\lambda)=\infty
$$

e portanto $\psi$ não está em $\Psi$. Seja $\varphi \in \Phi$, fazemos

$$
\varphi(A):=\frac{1}{2 \pi i} \int_{\Gamma} \varphi(-\lambda)(\lambda+A)^{-1} d \lambda=\frac{1}{2 \pi i} \int_{-\Gamma} \varphi(\lambda)(\lambda-A)^{-1} d \lambda,
$$

onde $\Gamma$ é qualquer curva simples em $\Sigma_{K} \backslash \mathbb{R}^{+}$suave por partes indo de $\infty \mathrm{e}^{-i \nu}$ até $\infty \mathrm{e}^{i \nu}$ para algum $\nu \in(0, \arcsin 1 /(2 K)]$. Segue de $(1.9),(1.10)$ e (1.11) e do Teorema de Cauchy que $\varphi(A)$ é bem definido em $L(E)$ e independente da escolha de $\Gamma$. Para mostrarmos que $\varphi(A)$ está bem definida, devemos mostrar que a integral converge. Como $\varphi \in \Phi$, temos que $\exists M>0$ tal que

$$
\forall \varepsilon>0,|\varphi(\lambda)| \leq \frac{\varepsilon}{|\lambda|^{\delta}} \quad \text { para }|\lambda|>M
$$


E então, se $\Gamma_{M}=\{\lambda \in \Gamma:|\lambda|>M\}$ temos:

$$
\begin{gathered}
\left\|\int_{\Gamma_{M}} \varphi(-\lambda)(\lambda+A)^{-1} d \lambda\right\| \leq \int_{\Gamma_{M}}\left|\varphi ( - \lambda ) \left\|\left|(\lambda+A)^{-1} \|\right| d \lambda \mid \leq\right.\right. \\
\leq \int_{\Gamma_{M}} \frac{\varepsilon}{|\lambda|^{\delta}} \frac{2 K+1}{1+\lambda}|d \lambda| \leq \varepsilon(2 K+1) \int_{\Gamma_{M}} \frac{1}{|\lambda|^{\delta+1}}|d \lambda| .
\end{gathered}
$$

A última integral converge, portanto segue que $\varphi(A)$ está bem definida.

Lema 1.5.2. A transformação $\Phi \rightarrow L(E), \varphi \mapsto \varphi(A)$ é um homomorfismo de álgebras.

Dado $z \in \mathbb{C}$, faça

$$
\varphi_{z}(\lambda):=\lambda^{z}=\mathrm{e}^{z \log \lambda}, \quad \lambda \in \mathbb{C} \backslash\left(-\mathbb{R}^{+}\right),
$$

está unicamente determinado, lembrando que $\log (z)$ é o ramo principal do logaritmo. Temos que $\varphi_{z} \in \Phi$ para $\operatorname{Re} z<0$; de fato pois se $\lambda=|\lambda| e^{i \arg (\lambda)} \operatorname{com}|\arg (\lambda)|<\pi$, então $\log (\lambda)=\log (|\lambda|)+i \arg (\lambda)$. Logo $\left|\lambda^{z}\right|=e^{\operatorname{Re}(z) \log (|\lambda|)-\operatorname{Im}(z) \arg (\lambda)}$. Com isso, temos que existe $\delta=\frac{-\operatorname{Re} z}{2}>0$ tal que

$$
\lim _{|\lambda| \rightarrow \infty}|\lambda|^{\delta} \psi(\lambda)=\lim _{|\lambda| \rightarrow \infty} e^{\frac{\operatorname{Rez} \log |\lambda|}{2}-\operatorname{Im}(z) \arg (\lambda)}=0 .
$$

Se $k=1,2,3, \cdots$ então $\varphi_{-k}$ é holomorfa em $\mathbb{C} \backslash\{0\}$ e podemos deformar $\Gamma$ para o círculo de raio $r$ centrado em 0 orientado positivamente, onde $0<r<1 /\left\|A^{-1}\right\|_{L(E)}$. Usando série de Neumann, temos que

$$
(\lambda-A)^{-1}=-A^{-1}\left(1-\lambda A^{-1}\right)^{-1}=-\sum_{j=0}^{\infty} A^{-j-1} \lambda^{j}, \quad|\lambda|=r,
$$

segue que

$$
\nu_{-k}(A)=\frac{-1}{2 \pi i} \int_{|\lambda|=r} \lambda^{-k}(\lambda-A)^{-1} d \lambda=-\operatorname{Res}\left(\lambda^{-k}(\lambda-A)^{-1}, 0\right)=A^{-k} .
$$

Isto justifica a seguinte definição de potências fracionárias de $A$ :

$$
A^{z}:=\varphi_{z}(A), \quad \operatorname{Re} z<0 .
$$

Se $0<\operatorname{Re} z<1$, podemos deformar $\Gamma$ sobre $\mathbb{R}^{+}$. Logo

$$
\begin{aligned}
A^{-z} & =\frac{1}{2 \pi i} \int_{\infty-i 0}^{0-i 0}(-\lambda)^{-z}(\lambda+A)^{-1} d \lambda+\frac{1}{2 \pi i} \int_{0+i 0}^{\infty+i 0}(-\lambda)^{-z}(\lambda+A)^{-1} d \lambda \\
& =-\frac{\mathrm{e}^{-i \pi z}}{2 \pi i} \int_{0}^{\infty} s^{-z}(s+A)^{-1} d s+\frac{\mathrm{e}^{i \pi z}}{2 \pi i} \int_{0}^{\infty} s^{-z}(s+A)^{-1} d s
\end{aligned}
$$


isto é

$$
A^{-z}=\frac{\sin \pi z}{\pi} \int_{0}^{\infty} s^{-z}(s+A)^{-1} d s, \quad 0<\operatorname{Re} z<1
$$

Aplicando a fórmula (1.21) ao caso $E:=\mathbb{C}$ e $A:=1$, em particular, segue que

$$
\int_{0}^{\infty} s^{-z}(1+s)^{-1} d s=\frac{\pi}{\sin \pi z}, \quad 0<\operatorname{Re} z<1
$$

Portanto deduzimos do fato que $A \in P_{K}(E)$ e da igualdade acima que

$$
\left\|A^{-z}\right\|_{L(E)} \leq K \frac{|\sin \pi z|}{\pi} \int_{0}^{\infty} s^{-\operatorname{Re} z}(1+s)^{-1} d s=K \frac{|\sin \pi z|}{\sin \pi \operatorname{Re} z}
$$

para $0<\operatorname{Re} z<1$. Agora não é difícil provar o seguinte resultado de continuidade:

Teorema 1.5.1. $\left\{A^{z} ; \operatorname{Re} z<0\right\} \cup\left\{A^{0}=I_{E}\right\}$ é um semigrupo fortemente contínuo $e$ holomorfo sobre $E$.

Prova: É uma consequência simples do teorema da derivação sob o sinal de integração que a aplicação $z \mapsto A^{z}$ é holomorfa em $\{z \in \mathbb{C}: \operatorname{Re} z<0\}$. Temos do Lema 1.5.2, que para $z_{1}, z_{2} \in \mathbb{C}$ com $\operatorname{Re}\left(z_{1}\right)<0$ e $\operatorname{Re}\left(z_{2}\right)<0$,

$$
\begin{aligned}
A^{z_{1}+z_{2}}=\varphi_{z_{1}+z_{2}}(A) & =\frac{1}{2 \pi i} \int_{\Gamma} \varphi_{z_{1}+z_{2}}(-\lambda)(\lambda+A)^{-1} d \lambda \\
& =\frac{1}{2 \pi i} \int_{\Gamma}(-\lambda)^{z_{1}+z_{2}}(-\lambda)(\lambda+A)^{-1} d \lambda \\
& =\frac{1}{2 \pi i} \int_{\Gamma}(-\lambda)^{z_{1}}(-\lambda)^{z_{2}}(-\lambda)(\lambda+A)^{-1} d \lambda \\
& =\frac{1}{2 \pi i} \int_{\Gamma} \varphi_{z_{1}}(-\lambda) \varphi_{z_{2}}(-\lambda)(\lambda+A)^{-1} d \lambda=\varphi_{z_{1}} \varphi_{z_{2}}(A) \\
& =\varphi_{z_{1}}(A) \varphi_{z_{2}}(A)=A^{z_{1}} A^{z_{2}}
\end{aligned}
$$

Resta mostrar que é fortemente contínuo em $z=0$.

Note que,

$$
(s+A)^{-1}-(1+s)^{-1} \supset(s+A)^{-1}\left(1-(s+A)(1+s)^{-1}\right)=(1+s)^{-1}(s+A)^{-1}(1-A)
$$


para $s>0$. Portanto, dado $e \in D(A)$ e $z$ com $0<\operatorname{Re} z<1$, segue de (1.21) e de (1.14) que

$$
\begin{aligned}
A^{-z} e-e & =\frac{\sin \pi z}{\pi} \int_{0}^{\infty} s^{-z}(s+A)^{-1} e d s-\frac{\sin \pi z}{\pi} \int_{0}^{\infty} s^{-z}(1+s)^{-1} e d s \\
& =\frac{\sin \pi z}{\pi} \int_{0}^{\infty} \frac{s^{-z}}{1+s}(s+A)^{-1}(1-A) e d s
\end{aligned}
$$

Consequentemente,

$$
\left\|A^{-z} e-e\right\|_{E} \leq K \frac{|\sin \pi z|}{\pi}\|(1-A) e\|_{E} \int_{0}^{\infty} \frac{s^{-\operatorname{Re} z}}{(1+s)^{2}} d s, \quad 0<\operatorname{Re} z<1
$$

Como a integral converge para 1 quando $\operatorname{Re} z \rightarrow 0$, vemos que $A^{-z} e \rightarrow e$, quando $z \rightarrow 0$ em $\{z \in \mathbb{C}:|\arg z| \leq \alpha\}$ para cada $\alpha \in(0, \pi / 2)$. Desde que $A^{-z}$ é uniformemente limitado para $z \in\{z \in \mathbb{C}:|\arg z| \leq \alpha\} \cap\{z \in \mathbb{C}: 0<\operatorname{Re} z<1\}$ para cada $\alpha \in(0, \pi / 2)$, graças a (1.14), $A^{z}$ converge para $I_{E}$ na topologia forte quando $z \rightarrow 0 \mathrm{em}\{z \in \mathbb{C}:|\arg z| \geq \pi / 2+\epsilon\}$ para cada $\epsilon \in(0, \pi / 2)$. Isto prova o teorema. $\square$

Já definimos $A^{z}$, quando $\operatorname{Rez}<0$. Agora mostraremos que $A^{z}$ é injetiva, para podermos definir as potências fracionárias para $\operatorname{Rez}>0$.

Suponha que $A^{z} e=0$ para algum $e \in E$ e $z \in \mathbb{C}$ com $\operatorname{Re} z<0$. Pelo Teorema 1.5.1, segue que $A^{z+w} e=A^{w} A^{z} e=0$ para $\operatorname{Re} w<0$. Portanto $A^{w} e=0$ para $\operatorname{Re} w<\operatorname{Re} z$. Em particular, $A^{-k} e=0$ para $k=0,1,2, \cdots \operatorname{com} k>-\operatorname{Re} z$. Consequentemente, $e=0$. Isto mostra que $A^{z}$ é injetiva para $\operatorname{Re} z<0$. Portanto podemos definir as potências fracionárias para $\operatorname{Re} z>0$ por

$$
A^{z}:=\left(A^{-z}\right)^{-1}, \quad \operatorname{Re} z>0
$$

Claro que, $A^{z} \in \mathcal{C}(E)$.

Teorema 1.5.2.

(i) Para $z, w \in \mathbb{C}$ com $0<\operatorname{Re} z<\operatorname{Re} w$ e e $\in D\left(A^{w}\right)$,temos que $D\left(A^{w}\right) \subset D\left(A^{z}\right)$.

(ii) $\overline{D\left(A^{z}\right)}=E, \quad \operatorname{Re} z>0$.

Prova: (i) Dados $z, w \in \mathbb{C}$ com $0<\operatorname{Re} z<\operatorname{Re} w$ e $e \in D\left(A^{w}\right)$, segue de

$$
e=A^{-w} A^{w} e=A^{-z-(w-z)} A^{w} e
$$


que $e \in D\left(A^{z}\right)$, isto é,

$$
D\left(A^{w}\right) \subset D\left(A^{z}\right), \quad 0<\operatorname{Re} z<\operatorname{Re} w .
$$

(ii) Dado $e \in D(A)$, faça $f:=A e$. Como $D(A)$ é denso em $E$, podemos encontrar para cada $\epsilon>0$ um elemento $u \in D(A)$ tal que $\|u-f\|_{E} \leq \epsilon /\left\|A^{-1}\right\|_{L(E)}$. Portanto, fazendo $v:=A u$,

$$
\left\|A^{-2} v-e\right\|_{E}=\left\|A^{-1} u-A^{-1} f\right\|_{E} \leq\left\|A^{-1}\right\|_{L(E)}\|u-f\|_{E} \leq \epsilon .
$$

Isto mostra que $\overline{D\left(A^{2}\right)} \supset D(A)$. Portanto $\overline{D\left(A^{2}\right)} \supset \overline{D(A)}=E$ o que garante que $D\left(A^{2}\right)$ é denso em $E$. Por indução vemos que $D\left(A^{k}\right)$ é denso em $E$ para $k=1,2,3, \cdots$. De fato, suponha que $\overline{D\left(A^{k}\right)}=E$. Sabemos que $\overline{D\left(A^{2}\right)}=E$ e $D\left(A^{k+1}\right) \subset D\left(A^{k}\right)$. Portanto $\overline{D\left(A^{k+1}\right)}=E$. Agora provaremos que $\overline{D\left(A^{z}\right)}=E, \quad \operatorname{Re} z>0$. Dado $z \in \mathbb{C}$ com $\operatorname{Rez}>0$, existe $k \geq 1$ tal que $0<\operatorname{Rez}<k$. E pelo Teorema 1.5.2 (i), $D\left(A^{k}\right) \subset D\left(A^{z}\right)$. Portanto segue que

$$
\overline{D\left(A^{z}\right)}=E, \quad \operatorname{Re} z>0,
$$

com isso concluímos a demonstração.

Teorema 1.5.3. Para $z, w \in \mathbb{C} c o m \operatorname{Re} z, \operatorname{Re} w, \operatorname{Re}(z+w) \neq 0$

$$
A^{z} A^{w} e=A^{z+w} e, \quad e \in D\left(A^{u}\right)
$$

onde $u \in\{z, w, z+w\}$ com $\operatorname{Re} u=\max \{\operatorname{Re} z, \operatorname{Re} w, \operatorname{Re}(z+w)\}$.

Prova: Suponha que $\operatorname{Re} z>0$ e $\operatorname{Re} w>0$. Dado

$$
e \in D\left(A^{z+w}\right) \subset D\left(A^{w}\right) \cap D\left(A^{z}\right)
$$

faça $f:=A^{z+w} e$. Então $e=A^{-(z+w)} f=A^{-w} A^{-z} f$ implica $A^{w} e=A^{-z} f$ o que, por sua vez, mostra que $f=A^{z} A^{w} e$; isto é,

$$
A^{z+w} e=A^{z} A^{w} e=A^{w} A^{z} e, \quad e \in D\left(A^{z+w}\right) .
$$

Se $\operatorname{Re} z>\operatorname{Re} w>0$ e $e \in D\left(A^{w}\right)$ então

$$
A^{-z} A^{w} e=A^{-(z-w)} A^{-w} A^{w} e=A^{-(z-w)} e=A^{w-z} e .
$$


Adicionalmente, se $e \in D\left(A^{z}\right)$ então, graças a identidade acima,

$$
A^{-w} A^{z} e=A^{-w} A^{w} A^{z-w} e=A^{z-w} e .
$$

Com isso concluímos que, dados $z, w \in \mathbb{C}$ com $\operatorname{Re} z, \operatorname{Re} w, \operatorname{Re}(z+w) \neq 0$,

$$
A^{z} A^{w} e=A^{z+w} e, \quad e \in D\left(A^{u}\right)
$$

onde $u \in\{z, w, z+w\}$ com $\operatorname{Re} u=\max \{\operatorname{Re} z, \operatorname{Re} w, \operatorname{Re}(z+w)\}$.

Considere a seguinte extensão de (1.21). Como usual o produto vazio é definido como sendo 1 .

Proposição 1.5.1. Suponha que $m=0,1,2, \cdots$ Temos que

$$
A^{-z}=\frac{\sin \pi z}{\pi} \frac{m !}{(1-z)(2-z) \cdots(m-z)} \int_{0}^{\infty} s^{m-z}(s+A)^{-m-1} d s
$$

para $0<\operatorname{Re} z<m+1$.

Prova: Suponha que $z$ satisfaz $0<\operatorname{Re} z<1$. Por (1.21) temos que:

$$
A^{-z}=\frac{\sin \pi z}{\pi} \int_{0}^{\infty} s^{-z}(s+A)^{-1} d s, \quad 0<\operatorname{Re} z<1
$$

Usando integração por partes na equação acima, temos:

$$
\begin{aligned}
A^{-z} & =\frac{\sin \pi z}{\pi(1-z)}\left[\left(\left.s^{1-z}(s+A)^{-1}\right|_{0} ^{\infty}+\int_{0}^{\infty} s^{1-z}(s+A)^{-2} d s\right]\right. \\
& =\frac{\sin \pi z}{\pi(1-z)} \int_{0}^{\infty} s^{1-z}(s+A)^{-2} d s
\end{aligned}
$$

Portanto (1.20) é válido para $m=1$. Suponhamos que (1.20) é válido para $m$, com $0<\operatorname{Re} z<1$, então temos

$$
A^{-z}=\frac{\sin \pi z}{\pi} \frac{m !}{(1-z)(2-z) \cdots(m-z)} \int_{0}^{\infty} s^{m-z}(s+A)^{-m-1} d s
$$

Provemos que vale para $m+1$; fazendo a integração por partes da integral hipótese indutiva, temos que:

$$
\int_{0}^{\infty} s^{m-z}(s+A)^{-m-1} d s=\frac{m+1}{m+1-z} \int_{0}^{\infty} s^{m+1-z}(s+A)^{-m+1-1} d s
$$


Logo temos que:

$$
A^{-z}=\frac{\sin \pi z}{\pi} \frac{(m+1) !}{(m-1) \cdots(m-z)(m+1-z)} \int_{0}^{\infty} s^{m+1-z}(s+A)^{-(m+1)-1} d s .
$$

Portanto a igualdade (1.20) vale para todo $m=0,1,2, \ldots$, com $0<\operatorname{Rez}<1$. Por (1.8) temos que a integral em (1.20) converge absolutamente para $0<\operatorname{Re} z<m+1$. De fato,

$$
\left\|\int_{0}^{\infty} s^{m-z}(s+A)^{-m-1} d s\right\| \leq k^{m+1} \int_{0}^{\infty} s^{m-\operatorname{Rez}}(1+s)^{-m-1} d s \leq k^{m+1} \int_{0}^{\infty} s^{m}(1+s)^{-m-1} d s
$$

E portanto o lado direito de (1.20) é uma aplicação holomorfa de $\{z \in \mathbb{C}: 0<\operatorname{Re} z<$ $m+1\}$ em $L(E)$. Sabemos pelo Teorema 1.5.1 que $A^{-z}$ é holomorfa para $0<\operatorname{Re}<1$. E ainda

$$
A^{-z}=\frac{\sin \pi z}{\pi} \frac{m !}{(1-z)(2-z) \cdots(m-z)} \int_{0}^{\infty} s^{m-z}(s+A)^{-m-1} d s,
$$

no intervalo $0<\operatorname{Rez}<1$, então vale $(1.20)$.

É uma consequência do Teorema 1.5.1 que $\left\{A^{-t} ; t \geq 0\right\}$ é um semigrupo fortemente contínuo sobre $E$. Denotamos o seu gerador infinitesimal por

$$
-\log A
$$

o que define o logarítimo de $A \in \mathcal{P}(E)$. Então a fórmula intuitiva

$$
A^{-t}=\mathrm{e}^{-t \log A}, \quad t \geq 0,
$$

é válida.

Definição 1.5.2. Um semigrupo analítico $\left\{\mathbf{e}^{-t B} ; t \geq 0\right\}$ é dito de ângulo $\alpha$, onde $0<$ $\alpha \leq \pi$, se existe uma função holomorfa

$$
T:\{z \in \mathbb{C}:|\arg z|<\alpha\} \rightarrow L(E)
$$

estendendo $\left\{\mathrm{e}^{-t B} ; t \geq 0\right\}$, tal que $T$ é fortemente contínuo no setor fechado $\{z \in \mathbb{C}$ : $|\arg z| \leq \alpha-\epsilon\} \cup\{0\}$ para cada $\epsilon \in(0, \alpha)$.

Dessa forma, $T$ é um semigrupo sobre $E$ e escrevemos $\mathrm{e}^{-z B}:=T(z)$ para $\{z \in \mathbb{C}$ : $|\arg z|<\alpha\} \cup\{0\}$. Esta notação é justificada já que $\frac{d}{d z} T(z)=-B T(z)$ para $z \in\{z \in$ $\mathbb{C}:|\arg z|<\alpha\}$. 
Teorema 1.5.4. Assuma que $A \in \mathcal{P}_{K}(E)$. Então $\left\{A^{-t} ; t \geq 0\right\}$ é um semigrupo analítico de ângulo $\pi / 2$. Adicionalmente,

$$
\left\|A^{-t}\right\|_{L(E)} \leq K^{m}, \quad 0 \leq t \leq m, \quad m=0,1,2, \cdots
$$

$e A^{-z}=\mathrm{e}^{-z \log A}$ para $\operatorname{Re} z<0$.

Prova: Pelo Teorema 1.5.1 a maior parte deste teorema já foi provado. Resta provar apenas a limitação. Aplicando a Proposição 1.5 .1 a $E:=\mathbb{C}$ e $A:=1$ vemos que

$$
\frac{\pi(1-t)(2-t) \cdots(m-t)}{m ! \sin \pi t}=\int_{0}^{\infty} s^{m-t}(1+s)^{-m-1} d s>0
$$

para $0<s<m+1$ e $m=0,1,2, \cdots$. Agora a Proposição 1.5.1 e (1.8) implicam

$$
\left\|A^{-t}\right\|_{L(E)} \leq K^{m+1} \frac{\sin \pi t}{\pi} \frac{m !}{(1-t)(2-t) \cdots(m-t)} \int_{0}^{\infty} s^{m-t}(1+s)^{-m-1} d t=K^{m+1}
$$

para $0<t<m+1$ e $m=0,1,2, \cdots$ a

Agora suponha que $-1<\operatorname{Re} z<1$. Entāo pomos

$$
A_{z} e:=\frac{\sin \pi z}{\pi z} \int_{0}^{\infty} s^{z}(s+A)^{-2} A e d s, \quad e \in D(A) .
$$

Observe que

$$
A_{0} e=\int_{0}^{\infty}(s+A)^{-2} d s A e=-\left.(s+A)^{-1} A e\right|_{0} ^{\infty}=e, \quad e \in D(A) .
$$

Adicionalmente, se $\operatorname{Re} z \neq 0$, segue de (1.21) e de (1.20) que

$$
A^{z} e=A^{z-1} A e=\frac{\sin \pi(1-z)}{\pi z} \int_{0}^{\infty} s^{z}(s+A)^{-2} A e d s=A_{z} e
$$

para $e \in D(A)$. Note que

$$
A^{-1} A_{z} \subset B_{z}:=\frac{\sin \pi z}{\pi z} \int_{0}^{\infty} s^{z}(s+A)^{-2} d s \in L(E) .
$$

Seja $\left(e_{j}\right)$ uma sequência em $D(A)$ tal que $e_{j} \rightarrow 0$ e $A_{z} e_{j} \rightarrow f$ em $E$. Então graças a (1.24), $B_{z} e_{j} \rightarrow 0$ e $B_{z} e_{j} \rightarrow A^{-1} f$ : o que implica que $f=0$. Portanto $A_{z}$ é fechável. Motivado por (1.22) e (1.23) fazemos

$$
A^{z}:=\text { fecho de } A_{z}, \quad \operatorname{Re} z=0
$$

Com estas consideraçōes já provamos a maior parte da seguinte afirmativa. 
Teorema 1.5.5. Suponha que $A \in P_{k}(E)$. Então a potência fracionária $A^{z}$ é; para cada $z \in \mathbb{C}$, um operador linear fechado densamente definido em $E$. Se $\operatorname{Re} z<0$, então $A^{z} \in L(E)$ e é dado pela integral

$$
A^{z}=\frac{1}{2 \pi i} \int_{\Gamma}(-\lambda)^{z}(\lambda+A)^{-1} d \lambda
$$

onde $\Gamma$ é qualquer curva simples suave por partes em $\mathbb{C} \backslash \mathbb{R}^{+}$indo de $\infty \mathrm{e}^{-i \varphi}$ a $\infty \mathrm{e}^{i \psi}$ para algum $\varphi, \psi \in(0, \pi)$ tal que $\sigma(-A)$ fica estritamente a esquerda de $\Gamma$. Adicionalmente,

(i) $A^{z}$ é a potência usual de A se $z$ é inteiro.

(ii) $A^{z} e=\frac{\sin \pi z}{\pi z} \int_{0}^{\infty} s^{z}(s+A)^{-2} A e d s, \quad e \in D(A), \quad-1<\operatorname{Re} z<1$.

(iii) Suponha que ou $m=0,1,2, \cdots, e \in D\left(A^{m}\right) e \max \{\operatorname{Re} z, \operatorname{Re} w\}<m$ ou $\operatorname{Re} z, \operatorname{Re} w$ $e \operatorname{Re}(z+w)$ não são nulos e $e \in D\left(A^{u}\right)$ onde $u \in\{z, w, z+w\}$, satisfaz $\operatorname{Re} u=$ $\max \{\operatorname{Re} z, \operatorname{Re} w, \operatorname{Re}(z+w)\}$. Então

$$
A^{z} A^{w} e=A^{z+w} e
$$

(iv) $A^{z} A^{w}=A^{z+w}$, $\operatorname{Re} z, \operatorname{Re} w>0$.

(v) $D\left(A^{w}\right) \stackrel{d}{\hookrightarrow} D\left(A^{z}\right) \stackrel{d}{\hookrightarrow} E, 0<\operatorname{Re} z<\operatorname{Re} w$, onde $D\left(A^{w}\right) \stackrel{d}{\hookrightarrow} D\left(A^{z}\right) \stackrel{d}{\hookrightarrow} E$ denota uma inclusão contínua e densa de $D\left(A^{w}\right)$ em $D\left(A^{z}\right)$.

(vi) $A^{z} \in \operatorname{Lis}\left(D\left(A^{z+w}\right), D\left(A^{w}\right)\right) \cap L i s\left(D\left(A^{z}\right), E\right), \operatorname{Re} z, \operatorname{Re} w>0$, onde Lis denota um isomorfismo linear.

(vii) Dada $m=0,1,2, \cdots$, a aplicação

$$
\{z \in \mathbb{C}: \operatorname{Re} z<m\} \rightarrow L\left(D\left(A^{m}\right), E\right), \quad z \mapsto A^{z}
$$

é holomorfa.

Note que, se $-A$ é o gerador infinitesimal de um $C_{0}$-semigrupo com decaimento exponencial em $E$, então $A$ é do tipo positivo. Neste caso, podemos obter outra fórmula de representação útil para $A^{z} \operatorname{com} \operatorname{Re} z>0$. 
Teorema 1.5.6. Suponha que $A$ é o gerador de um $C_{0}$-semigrupo com decaimento exponencial. Então

$$
A^{-z}=\frac{1}{\Gamma(z)} \int_{0}^{\infty} t^{z-1} \mathrm{e}^{-t A} d t, \quad \operatorname{Re} z>0 .
$$

Prova: Pela limitação exponencial, temos que

$$
\left\|\int_{0}^{\infty} t^{z-1} \mathrm{e}^{-t A} d t\right\|_{L(E)} \leq M \int_{0}^{\infty} t^{\mathrm{Rez}-1} \mathrm{e}^{-\sigma t} d t
$$

e das propriedades conhecidas da função $\Gamma$ segue que a aplicação

$$
\{z \in \mathbb{C}: \operatorname{Re} z>0\} \rightarrow L(E), \quad z \mapsto \frac{1}{\Gamma(z)} \int_{0}^{\infty} t^{z-1} \mathrm{e}^{-t A} d t
$$

é holomorfa. E pelo Teorema 1.5.1, temos que $A^{z}$ para Rez $<0$ é holomorfo. Portanto é suficiente provar a igualdade para $0<z<1$.

Pela Proposição 1.5.1, dado $z \in(0,1)$,

$$
A^{-z}=\frac{\sin \pi z}{\pi} \int_{0}^{\infty} s^{-z}(s+A)^{-1} d s
$$

Por outro lado, sabemos da teoria de semigrupos que

$$
(s+A)^{-1}=\int_{0}^{\infty} \mathrm{e}^{-s t} \mathrm{e}^{-t A} d t, \quad s>0 .
$$

Pelo Teorema de Fubini

$$
\begin{aligned}
A^{-z} & =\frac{\sin \pi z}{\pi} \int_{0}^{\infty} s^{-z} \int_{0}^{\infty} \mathrm{e}^{-s t} \mathrm{e}^{-t A} d t d s=\frac{\sin \pi z}{\pi} \int_{0}^{\infty} \mathrm{e}^{-t A} \int_{0}^{\infty} s^{-z} \mathrm{e}^{-t s} d s d t \\
& =\frac{\sin \pi z}{\pi} \Gamma(1-z) \int_{0}^{\infty} t^{z-1} \mathrm{e}^{-t A} d t .
\end{aligned}
$$

Portanto a afirmativa segue da fórmula

$$
\Gamma(z) \Gamma(1-z)=\pi / \sin \pi z \cdot \square
$$

Teorema 1.5.7. Suponha que $A \in \mathcal{P}_{K}\left(E_{0}\right)$ e $0<\alpha<1$, então

$$
A^{-\alpha}=\frac{\sin \pi \alpha}{\pi} \int_{0}^{\infty} s^{-\alpha}(s+A)^{-1} d s
$$

$e$

$$
\left\|(\mu+A)^{-1} e\right\|_{E_{0}} \leq K \mu^{\alpha-1}\left\|A^{-\alpha} e\right\|_{E_{0}}, \quad \mu>0, e \in E_{0} .
$$

Aqui $K$ é uma constante dependendo de $A$ e a última desigualdade vale para $0 \leq \alpha \leq 1$. 
Prova: Por (1.8), sabemos que $\left\|s(s+A)^{-1}\right\|_{L\left(E_{0}\right)} \leq K,\left\|A(s+A)^{-1}\right\|_{L\left(E_{0}\right)} \leq K+1, s \geq 0$. Seja $e \in D(A)$, então usando a proposiçào 1.5.1, temos

$$
\begin{aligned}
(\mu+A)^{-1} e & =A^{\alpha-1} A(\mu+A)^{-1} A^{-\alpha} e \\
& =\frac{\sin \pi \alpha}{\pi} \int_{0}^{\infty} s^{\alpha-1} A(\mu+A)^{-1}(s+A)^{-1} A^{-\alpha} e d s .
\end{aligned}
$$

Portanto

$$
\begin{aligned}
\left\|(\mu+A)^{-1} e\right\|_{E_{0}} & \leq \frac{\sin \pi \alpha}{\pi} K(K+1)\left[\int_{0}^{\mu} s^{\alpha-1} d s \mu^{-1}+\int_{\mu}^{\infty} s^{\alpha-2} d s\right]\left\|A^{-\alpha} e\right\|_{E_{0}} \\
& \leq K(K+1) \frac{\sin \pi \alpha}{\pi}\left[\frac{1}{\alpha} \mu^{\alpha-1}+\frac{1}{\Gamma-\alpha} \mu^{\alpha-1}\right]\left\|A^{-\alpha} e\right\|_{E_{0}}
\end{aligned}
$$

e o resultado segue.

Teorema 1.5.8.

1. Assuma que $A \in \mathcal{P}_{K}\left(E_{0}\right)$ e que $e \in D\left(A^{\alpha}\right)$ para algum $\alpha, 0<\alpha \leq 1$. Então, se $e_{\epsilon}=(I+\epsilon A)^{-1} e, \epsilon>0$, temos que

$$
\begin{gathered}
\left\|e_{\epsilon}-e\right\|_{E_{0}} \leq K \epsilon^{\alpha}\left\|A^{\alpha} e\right\|_{E_{0}} \\
\left\|A e_{\epsilon}\right\|_{E_{0}} \leq K \epsilon^{\alpha-1}\left\|A^{\alpha} e\right\|_{E_{0}}
\end{gathered}
$$

para todo $\epsilon>0$.

2. Suponha que $e \in E_{0}$ e que para algum $\alpha, 0<\alpha \leq 1,\|e\|_{E_{0}}<B<\infty$, existe $e_{\epsilon} \in D(A)$, para todo $\epsilon>0$ tal que

$$
\begin{aligned}
& \left\|e_{\epsilon}-e\right\|_{E_{0}} \leq B \epsilon^{\alpha}, \quad \forall \epsilon>0 \\
& \left\|A e_{\epsilon}\right\|_{E_{0}} \leq B \epsilon^{\alpha-1}, \quad \forall \epsilon>0 .
\end{aligned}
$$

Então $e \in D\left(A^{\beta}\right)$ para qualquer $\beta$ em $0<\beta<\alpha e$

$$
\left\|A^{\beta} e\right\|_{E_{0}} \leq K_{\alpha, \beta} B
$$

para uma constante $K_{\alpha, \beta}$ dependendo somente de $A, \alpha$ e $\beta$. 
Prova: 1) Pelo Teorema 1.5.7

$$
\begin{aligned}
\left\|A e_{\epsilon}\right\|_{E_{0}} & =\left\|A^{1-\alpha}(1+\epsilon A)^{-1} A^{\alpha} e\right\|_{E_{0}} \\
& \leq K \epsilon^{\alpha-1}\left\|A^{\alpha} e\right\|_{E_{0}}
\end{aligned}
$$

e portanto $\left\|e_{\epsilon}-e\right\|_{E_{0}}=\left\|\epsilon A(I+\epsilon A)^{-1} e\right\|_{E_{0}} \leq K \epsilon^{\alpha}\left\|A^{\alpha} e\right\|_{E_{0}}$.

2) Para qualquer $\mu>0, \epsilon>0$

$$
\begin{aligned}
\left\|A(\mu+A)^{-1} e\right\|_{E_{0}} & \leq\left\|A(\mu+A)^{-1}\left(e-e_{\epsilon}\right)\right\|_{E_{0}}+\left\|(\mu+A)^{-1} A e_{\epsilon}\right\|_{E_{0}} \\
& \leq(K+1) B \epsilon^{\alpha}+K \mu^{-1} B \epsilon^{\alpha-1} .
\end{aligned}
$$

Logo, escolhendo $\epsilon=\mu^{-1}$

$$
\left\|A(\mu+A)^{-1} e\right\|_{E_{0}} \leq B(2 K+1) \mu^{-\alpha}
$$

e claramente

$$
\left\|A(\mu+A)^{-1} e\right\|_{E_{0}} \leq(K+1)\|e\|_{E_{0}} \leq B(2 K+1) .
$$

Logo

$$
\left\|A(\mu+A)^{-1} e\right\|_{E_{0}} \leq B(2 K+1) \min \left\{1, \mu^{-\alpha}\right\} .
$$

Se $0<\beta<\alpha$ segue que $\int_{0}^{\infty}\left\|s^{\beta-1} A(s+A)^{-1} e\right\|_{E_{0}} d s<\infty \mathrm{e}$

$$
J_{\beta} e=\frac{\sin \pi \beta}{\pi} \int_{0}^{\infty} s^{\beta-1} A(s+A)^{-1} e d s
$$

é tal que $\left\|J_{\beta} e\right\|_{E_{0}} \leq K_{\alpha, \beta} B$, mas

$$
f_{R}=\frac{\sin \pi \beta}{\pi} \int_{0}^{R} s^{\beta-1}(s+A)^{-1} e d s \rightarrow A^{\beta-1} e
$$

quando $R \rightarrow \infty$ e $A f_{R} \rightarrow J_{\beta}$ e quando $R \rightarrow \infty$. Como $A$ é fechado segue que $A^{\beta-1} e \in$ $D(A)$ o que significa $e \in D\left(A^{\beta}\right)$, desde que $e=A^{-\beta}\left(A A^{\beta-1} e\right)$, e $\left\|A^{\beta} e\right\|_{E_{0}}=\left\|J_{\beta} e\right\|_{E_{0}} \leq$ $K_{\alpha, \beta} B_{\cdot \square}$

Corolário 1.5.1. Se e $\in D\left(A^{\alpha}\right), \alpha>0$ e $0<\beta<\alpha$ então

$$
A^{\beta} e=\frac{\sin \pi \beta}{\pi} \int_{0}^{\infty} s^{\beta-1} A(s+A)^{-1} e d s .
$$


Teorema 1.5.9. Existe uma constante $K$ dependendo somente de $A$, tal que $\left\|A^{\alpha} e\right\|_{E_{0}} \leq$ $K\|A e\|_{E_{0}}^{\alpha}\|e\|_{E_{0}}^{1-\alpha}$ para $0 \leq \alpha \leq 1, e \in D(A)$.

Prova: Para $\alpha=0$ e para $\alpha=1$ é fácil verificar. Como mostrado no Corolário 1.5.1 para $0<\alpha<1, e \in D(A)$

$$
A^{\alpha} e=\frac{\sin \pi \alpha}{\pi} \int_{0}^{\infty} s^{\alpha-1} A(s+A)^{-1} e d s
$$

$\log 0$

$$
\begin{aligned}
\left\|A^{\alpha} e\right\|_{E_{0}} & \leq \frac{\sin \pi \alpha}{\pi}\left[\int_{0}^{\mu} s^{\alpha-1}(K+1)\|e\| d s+\int_{\mu}^{\infty} s^{\alpha-2} K\|A e\| d s\right] \\
& \leq \frac{\sin \pi \alpha}{\pi}(K+1)\left[\frac{\mu^{\alpha}}{\alpha}\|e\|_{E_{0}}+\frac{\mu^{\alpha-1}}{1-\alpha}\|A e\|_{E_{0}}\right]
\end{aligned}
$$

para qualquer $\mu>0$. Seja $\mu=\|A e\|_{E_{0}} /\|e\|_{E_{0}}$. Então

$$
\left\|A^{\alpha} e\right\|_{E_{0}} \leq(K+1) \frac{\sin \pi \alpha}{\pi}\left[\frac{1}{\alpha}+\frac{1}{1-\alpha}\right]\|A e\|_{E_{0}}^{\alpha}\|e\|_{E_{0}}^{1-\alpha}
$$

e a constante é uniformemente limitada para $0<\alpha<1$.

Corolário 1.5.2. Seja $A \in P_{K}\left(E_{0}\right)$ e $B: D(B) \subset E_{0} \rightarrow E_{0}$ um operador fechado tal que $D(B) \supset D\left(A^{\alpha}\right)$, para algum $\alpha>0$. Então existem constantes $C, C_{1}>0$ tais que

$$
\|B e\|_{E_{0}} \leq C\left\|A^{\alpha} e\right\|_{E_{0}}, \quad e \in D\left(A^{\alpha}\right)
$$

$e$

$$
\|B e\|_{E_{0}} \leq C_{1}\left(\mu^{\alpha}\|e\|_{E_{0}}+\mu^{\alpha-1}\|A e\|_{E_{0}}\right), \quad \mu>0, e \in D(A) .
$$

Agora, consideramos o caso em que $A$ é setorial; isto é, $\left\{\mathrm{e}^{-A t}, t \geq 0\right\}$ é semigrupo analítico.

Teorema 1.5.10. Assuma que A é setorial. Logo $\left\{\mathrm{e}^{-A t} ; t \geq 0\right\}$ é um semigrupo analítico, suponha que $\rho(A) \supset(-\infty, 0]$. Então

1. Se $t>0, \alpha \geq 0, R\left(\mathrm{e}^{-A t}\right) \subset D\left(A^{\alpha}\right) e$

$$
\left\|A^{\alpha} \mathrm{e}^{-A t}\right\|_{L\left(E_{0}\right)} \leq M_{\alpha} t^{-\alpha}, \quad 0<t \leq 1,
$$

$\alpha \mapsto M_{\alpha}$ é contínua em $[0, \infty)$.

2. Se $\alpha>0$, temos que $t^{\alpha} A^{\alpha} \mathrm{e}^{-A t} e \rightarrow 0$ quando $t \rightarrow 0^{+}$para cada $e \in E_{0}$. 
3. $\left\|\left(\mathrm{e}^{-A t}-I\right) A^{-\alpha}\right\|_{L\left(E_{0}\right)} \leq M_{1-\alpha} \frac{t^{\alpha}}{\alpha}$ se $0<\alpha \leq 1,0 \leq t \leq 1$.

Prova: 1) Se $t>0, R\left(\mathrm{e}^{-A t}\right) \subset D(A)$ e $\left\|A \mathrm{e}^{-A t}\right\|_{L\left(E_{0}\right)} \leq M t^{-1},\left\|\mathrm{e}^{-A t}\right\|_{L\left(E_{0}\right)} \leq M$ para $0 \leq t \leq 1$. Logo, para qualquer inteiro $m, R\left(\mathrm{e}^{-A t}\right) \subset D\left(A^{m}\right)$ pois $\mathrm{e}^{-A t / m}$ leva $E_{0}$ em $D(A)$ e $D\left(A^{k}\right)$ em $D\left(A^{k+1}\right)$, logo $\mathbf{e}^{-A t}=\left(\mathrm{e}^{-A t / m}\right)^{m}$ leva $E_{0}$ em $D(A)$ em $D\left(A^{2}\right)$ em $\cdots$ em $D\left(A^{m}\right)$ e $0 \leq \alpha \leq 1$

$$
\left\|A^{\alpha} \mathrm{e}^{-A t}\right\|_{L\left(E_{0}\right)} \leq K\left\|A \mathrm{e}^{-A t}\right\|_{L\left(E_{0}\right)}^{\alpha}\left\|\mathrm{e}^{-A t}\right\|_{L\left(E_{0}\right)}^{1-\alpha} \leq K M t^{-\alpha}
$$

logo para $m=0,1,2, \cdots, 0 \leq \alpha \leq 1,0<t \leq 1$

$$
\begin{aligned}
\left\|A^{m+\alpha} \mathrm{e}^{-A t}\right\|_{L\left(E_{0}\right)} & \leq\left\|A^{\alpha} \mathrm{e}^{-A t /(m+1)}\right\|_{L\left(E_{0}\right)}\left\|A \mathrm{e}^{-A t /(m+1)}\right\|_{L\left(E_{0}\right)}^{m} \\
& \leq K M^{m+1}(m+1)^{m+\alpha} t^{-m-\alpha}
\end{aligned}
$$

2) Se $e \in D\left(A^{m}\right)$ para algum $m \geq \alpha>0, t^{\alpha} A^{\alpha} \mathrm{e}^{-A t} e \rightarrow 0$ quando $t \rightarrow 0^{+} \mathrm{e}$ $\left\|t^{\alpha} A^{\alpha} \mathrm{e}^{-A t}\right\|_{L\left(E_{0}\right)} \leq M_{\alpha}$ para $0<t \leq 1$, logo o resultado vale para todo $e \in E_{0}$.

3) Para todo $e \in E_{0}$ temos que

$$
\left\|\left(\mathrm{e}^{-A t}-I\right) \cdot A^{-\alpha} e\right\|_{E_{0}}=\left\|-\int_{0}^{t} A^{1-\alpha} \mathrm{e}^{-A s} e d s\right\|_{E_{0}} \leq \int_{0}^{t} M_{1-\alpha} s^{\alpha-1}\|e\|_{E_{0}} d s \cdot \square
$$

\subsection{Problema de Cauchy Não Homogêneo}

O objetivo deste capítulo é o estudo da existência de soluções de problemas de Cauchy (problemas de valor inicial) para equações lineares não homogêneas da forma

$$
\begin{aligned}
& \frac{d}{d t} e=A e+f(t), \quad t_{0}<t<t_{1} \\
& e\left(t_{0}\right)=e_{0} \in E_{0}
\end{aligned}
$$

onde $A$ é o gerador de um semigrupo fortemente contínuo $\left\{\mathbf{e}^{A t} ; t \geq 0\right\} \subset L\left(E_{0}\right)$ e $f$ : $\left[t_{0}, t_{1}\right) \rightarrow E_{0}$ é contínua por partes e contínua a direita.

\section{Definição 1.6.1.}

a) Uma função contínua e $\left[t_{0}, t_{1}\right) \rightarrow E_{0}$ é uma solução forte de (1.26) se e $\left(t_{0}\right)=e_{0}$ e para $t_{0}<t<t_{1}, e(t) \in D(A), \frac{d^{+}}{d t} e(t)=\lim _{h \rightarrow 0^{+}} \frac{e(t+h)-e(t)}{h}$ existe, (1.26) 
é satisfeito com $\frac{d}{d t} e(t)$ subsizituido por $\frac{d^{+}}{d t} e(t)$ e $t \mapsto \frac{d^{+}}{d t} e(t)$ é contínua onde $f$ é contínua. Note que se $t \mapsto f(t)$ é uma função contínua e $t \mapsto e(t)$ é uma solução forte de (1.26) então $t \mapsto e(t)$ é continuamente diferenciável e (1.26) se verifica para cada $t \in\left(t_{0}, t_{1}\right)$.

b) Uma função contínua $e:\left[t_{0}, t_{1}\right) \rightarrow E_{0}$ é uma solução fraca de (1.26) em $\left[t_{0}, t_{1}\right)$ se $e\left(t_{0}\right)=e_{0} \in E_{0}$ e para todo $e^{*} \in D\left(A^{*}\right), t \mapsto\left\langle e^{*}, e(t)\right\rangle$ tem derivada a direita $e$

$$
\frac{d^{+}}{d t}\left\langle e^{*}, e(t)\right\rangle=\left\langle A^{*} e^{*}, e(t)\right\rangle+\left\langle e^{*}, f(t)\right\rangle, \quad t_{0}<t<t_{1}
$$

Note que, se $t \mapsto\left\langle e^{*}, f(t)\right\rangle$ é uma função contínua e $t \mapsto e(t)$ é uma solução fraca de (1.26), então $t \mapsto\left\langle e^{*}, e(t)\right\rangle$ é continuamente diferenciável e (1.27) se verifica com $\frac{d^{+}}{d t}$ substituido por $\frac{d}{d t}$.

Definiçāo 1.6.2. Um subconjunto $S^{*} \subset E_{0}^{*}$ é dito total se: $e \in E_{0},\left\langle e^{*}, e\right\rangle=0, \forall e^{*} \in S^{*}$ implica $e=0$.

Lema 1.6.1. Se $A: D(A) \subset E_{0} \rightarrow E_{0}$ é fechado e densamente definido, então $D\left(A^{*}\right)$ é total.

Prova: Seja $e \in E_{0}$ tal que $\left\langle e^{*}, e\right\rangle=0$ para todo $e^{*} \in D\left(A^{*}\right)$. Queremos mostrar que $e=0$. Como o gráfico de $A^{*}, G\left(A^{*}\right)=\left\{\left(e^{*}, A^{*} e^{*}\right): e^{*} \in D\left(A^{*}\right)\right\}$ é o anulador em $E_{0}^{*} \times E_{0}^{*}$ de $\Gamma=\{(-A e, e): e \in D(A)\} ;$ isto é, $G\left(A^{*}\right)=\Gamma^{\perp}$. Note que $G\left(A^{*}\right)$ também anula $(e, 0)$, assim segue que $(e, 0) \in G\left(A^{*}\right)^{\perp}=\Gamma$ e portanto $e=0$

Através do teorema a seguir obtemos formas de manuseio mais simples pára as soluções fracas e estabelecemos algumas relações importantes entre soluções fracas $\ell$ fortes.

\section{Teorema 1.6.1.}

1. Toda solução forte de (1.26) é também uma solução fraca de (1.26).'

2. Se $e:\left[t_{0}, t_{1}\right) \rightarrow E_{0}$ é uma solução fraca de (1.26), então

$$
e(t)=\mathrm{e}^{A\left(t-t_{0}\right)} e_{0}+\int_{t_{0}}^{t} \mathrm{e}^{A(t-s)} f(s) d s, \quad t_{0} \leq t<t_{1} .
$$

Em particular, existe uma única solução fraca de (1.26). 
3. Se e $:\left[t_{0}, t_{1}\right) \rightarrow E_{0}$ é definido por (1.28), então e $:\left[t_{0}, t_{1}\right) \rightarrow E_{0}$ é uma solução fraca de (1.26).

4. Se e $:\left[t_{0}, t_{1}\right) \rightarrow E_{0}$ é uma solução fraca e para algum $t \in\left(t_{0}, t_{1}\right)$ ou $e(t) \in D(A)$ ou $\frac{d^{+}}{d t} e(t)$ existe, então ambos são verdadeiros e para este instante

$$
\frac{d^{+}}{d t} e(t)=A e(t)+f(t)
$$

Prova: A prova de 1) é simples. Se $e:\left[t_{0}, t_{1}\right) \rightarrow E_{0}$ é uma solução forte de (1.26) em $\left[t_{0}, t_{1}\right)$ então $e\left(t_{0}\right)=e_{0} \in E_{0}$ e para todo $e^{*} \in D\left(A^{*}\right)$

$$
\begin{aligned}
& \frac{d^{+}}{d t}\left\langle e^{*}, e(t)\right\rangle=\lim _{h \rightarrow 0^{+}} \frac{\left\langle e^{*}, e(t+h)-e(t)\right)}{h}=\left\langle e^{*}, \lim _{h \rightarrow 0^{+}} \frac{e(t+h)-e(t)}{h}\right\rangle= \\
= & \left\langle e^{*}, \frac{d^{+}}{d t} e(t)\right\rangle=\left\langle e^{*}, A e(t)+f(t)\right\rangle=\left\langle A^{*} e^{*}, e(t)\right\rangle+\left\langle e^{*}, f(t)\right\rangle, \quad t_{0}<t<t_{1} .
\end{aligned}
$$

Portanto $e:\left[t_{0}, t_{1}\right) \rightarrow E_{0}$ é uma solução fraca de (1.26).

Provaremos agora o item 3), pois usaremos ele na prova de 2).

Defina $e:\left[t_{0}, t_{1}\right) \rightarrow E_{0}$ por (1.28) e seja $e^{*} \in D\left(A^{*}\right)$. Para qualquer $e \in D(A)$, temos que:

$$
\frac{d}{d s} e^{A s} e=A e^{A s} e
$$

pelo Teorema 1.1.3, (1). Assim, integrando a igualdade acima temos:

$$
e^{A t} e-e=\int_{0}^{t} A e^{A s} e d s
$$

Portanto

$$
\left\langle e^{*}, \mathrm{e}^{A t} e\right)-\left\langle e^{*}, e\right\rangle=\int_{0}^{t}\left\langle A^{*} e^{*}, \mathrm{e}^{A s} e\right) d s
$$

para $e \in D(A)$ e por continuidade para todo $e \in E_{0}$. Logo, para qualquer $e \in E_{0}, t \mapsto$ $\left\langle e^{*}, \mathrm{e}^{A t} e\right\rangle$ é diferenciável com derivada $\left\langle A^{*} e^{*}, \mathrm{e}^{A t} e\right\rangle$. Usando isto calculamos $\frac{d^{+}}{d t}\left\langle e^{*}, e(t)\right)$ e vemos que $e:\left[t_{0}, t_{1}\right) \rightarrow E_{0}$ é uma solução fraca; de fato,

$$
\begin{aligned}
\frac{d^{+}}{d t}\left\langle e^{*}, e(t)\right)= & \lim _{h \rightarrow 0^{+}} \frac{\left\langle e^{*}, e(t+h)-e(t)\right\rangle}{h} \\
= & \lim _{h \rightarrow 0^{+}} \frac{\left\langle e^{*}, e^{A\left[\left(t-t_{0}\right)+h\right]} e_{0}-e^{A\left(t-t_{0}\right)} e_{0}\right\rangle}{h} \\
& +\lim _{h \rightarrow 0^{+}} \frac{\left\langle e^{*}, \int_{t_{0}}^{t+h} e^{A(t+h-s)} f(s) d s-\int_{t_{0}}^{t} e^{A(t-s)} f(s) d s\right\rangle}{h}
\end{aligned}
$$




$$
\begin{aligned}
= & \frac{d}{d t}\left\langle e^{*}, e^{A\left(t-t_{0}\right)} e\right\rangle+\frac{d}{d t}\left\langle e^{*}, \int_{t_{0}}^{t} e^{A(t-s)} f(s) d s\right\rangle \\
= & \left\langle A^{*} e^{*}, e^{A\left(t-t_{0}\right)} e\right\rangle+\lim _{h \rightarrow 0^{+}} \frac{1}{h}\left\langle e^{*}, \int_{t_{0}}^{t+h} \mathrm{e}^{A(t+h-s)} f(s) d s\right\rangle \\
& -\lim _{h \rightarrow 0^{+}} \frac{1}{h}\left(e^{*}, \int_{t_{0}}^{t} \mathrm{e}^{A(t-s)} f(s) d s\right\rangle \\
= & \left\langle A^{*} e^{*}, e^{A\left(t-t_{0}\right)} e\right\rangle+\lim _{h \rightarrow 0^{+}} \frac{1}{h}\left\langle e^{*}, \int_{t}^{t+h} \mathrm{e}^{A(t+h-s)} f(s) d s\right\rangle \\
& +\lim _{h \rightarrow 0^{+}} \frac{1}{h}\left(e^{*},\left(\mathrm{e}^{A h}-I\right) \int_{t_{0}}^{t} \mathrm{e}^{A(t-s)} f(s) d s\right\rangle \\
= & \left\langle A^{*} e^{*}, e^{A\left(t-t_{0}\right)} e\right\rangle+\left\langle e^{*}, f(t)\right\rangle+\left\langle A^{*} e^{*}, \int_{t_{0}}^{t} \mathrm{e}^{A(t-s)} f(s) d s\right\rangle \\
= & \left\langle A^{*} e^{*}, e(t)\right\rangle+\left\langle e^{*}, f(t)\right\rangle .
\end{aligned}
$$

Prova de 2). Se existem duas soluções de (1.26), a diferença entre elas $u:\left[t_{0}, t_{1}\right) \rightarrow E_{0}$ é uma função contínua com $u\left(t_{0}\right)=0 \mathrm{e} \frac{d^{+}}{d t}\left\langle e^{*}, u(t)\right)=\left\langle A^{*} e^{*}, u(t)\right), t_{0} \leq t<t_{1}$ e $e^{*} \in D\left(A^{*}\right)$. Integrando a igualdade acima de $t_{0}$ à $t$, obtemos:

$$
\left\langle e^{*}, u(t)\right\rangle=\int_{t_{0}}^{t}\left\langle A^{*} e^{*}, u(s)\right\rangle d s
$$

É conveniente trabalhar com uma função $C^{1}$, logo seja $U(t)=\int_{t_{0}}^{t} u(s) d s ;$ então $\left(e^{*}, \frac{d}{d t} U(t)\right\rangle=$ $\left\langle A^{*} e^{*}, U(t)\right)$.

Note que $\left(\mathrm{e}^{A t}\right)^{*} D\left(A^{*}\right) \subset D\left(A^{*}\right)$ para $t \geq 0$, já que $\left\langle\left(\mathrm{e}^{A t}\right)^{*} e^{*}, A e\right\rangle=\left\langle A^{*} e^{*}, \mathrm{e}^{A t} e\right\rangle$ para $e^{*} \in D\left(A^{*}\right), e \in D(A)$. Logo, para qualquer $t^{*} \in\left(t_{0}, t_{1}\right)$

$$
\frac{d}{d t}\left\langle e^{*}, \mathrm{e}^{A\left(t^{*}-t\right)} U(t)\right)=\left\langle e^{*} \mathrm{e}^{A\left(t^{*}-t\right)} \frac{d}{d t} U(t)\right\rangle-\left(A^{*} e^{*}, \mathrm{e}^{A\left(t^{*}-t\right)} U(t)\right\rangle=0
$$

para $t_{0} \leq t \leq t^{*}$

Como $U\left(t_{0}\right)=0,\left\langle e^{*}, U\left(t^{*}\right)\right)=0$ para todo $e^{*} \in D\left(A^{*}\right)$, portanto $U\left(t^{*}\right)=0$ (pois $D\left(A^{*}\right)$ é total). E como $t^{*}$ é arbitrário, segue que $u(s)=0$ para $t_{0} \leq s \leq t_{1}$. Dessa forma, por 3) e pela unicidade de soluçōes fracas, concluímos que se $e:\left[t_{0}, t_{1}\right) \rightarrow E_{0}$ é uma solução fraca de (1.26), então vale (1.28).

Prova de 4). Se $e(\cdot)$ é uma solução fraca, dada por (1.28), então para $t_{0} \leq t<t+h<t_{1}$

$$
\frac{e(t+h)-e(t)}{h}=\frac{1}{h} \int_{t}^{t+h} \mathrm{e}^{A(t+h-s)} f(s) d s+\frac{1}{h}\left(\mathrm{e}^{A h}-I\right) e(t) .
$$

O termo do meio converge para $f\left(t^{+}\right)=f(t)$ quando $h \rightarrow 0^{+}$, logo se um dos outros termos converge, ambos devem convergir. $\square$ 
A seguir damos condições simples que asseguram a diferenciabilidade de uma solução fraca.

Teorema 1.6.2. Suponha que $A$ ef são como antes e seja e: $\left[t_{0}, t_{1}\right) \rightarrow E_{0}$ é uma solução fraca de (1.26). Se $e_{0} \in D(A)$ e ou

1. $f(t) \in D(A)$ com $t \mapsto A f(t) \in E_{0}$ contínua a direita em $\left[t_{0}, t_{1}\right)$ ou

2. $\frac{d^{+}}{d t} f(t)=\dot{f}(t)$ existe e é contínua a direita em $\left[t_{0}, t_{1}\right)$

então $\frac{d^{+}}{d t} e(t)$ existe, $e(t) \in D(A)$ e e $:\left[t_{0}, t_{1}\right) \rightarrow E_{0}$ é uma solução forte.

Prova: Seja $u(t)=\int_{t_{0}}^{t} \mathrm{e}^{A(t-s)} f(s) d s$, então $e(t)=\mathrm{e}^{A\left(t-t_{0}\right)} e_{0}+u(t)$. Como $e_{0} \in D(A)$, $t \mapsto \mathrm{e}^{A\left(t-t_{0}\right)} e_{0}$ é $C^{1}$, então basta mostrarmos que se $t_{0} \leq t<t+h<t_{1}, \exists \frac{d^{+}}{d t} u(t)$. De fato, se $t_{0} \leq t<t+h<t_{1}$

$$
\frac{u(t+h)-u(t)}{h}=\frac{1}{h} \int_{t}^{t+h} \mathrm{e}^{A(t+h-s)} f(s) d s+\int_{t_{0}}^{t} \mathrm{e}^{A(t-s)} \frac{\mathrm{e}^{A h}-I}{h} f(s) d s
$$

e por (1), temos que

$$
\frac{d^{+}}{d t} u(t)=f(t)+\int_{t_{0}}^{t} \mathrm{e}^{A(t-s)} A f(s) d s
$$

Por outro lado, temos:

$$
\frac{u(t+h)-u(t)}{h}=\frac{1}{h} \int_{t_{0}}^{t_{0}+h} \mathrm{e}^{A(t+h-s)} f(s) d s+\int_{t_{0}}^{t} \mathrm{e}^{A(t-s)} \frac{f(s+h)-f(s)}{h} f(s) d s
$$

e usando (2), segue que:

$$
\frac{d^{+}}{d t} u(t)=\mathrm{e}^{A\left(t-t_{0}\right)} f\left(t_{0}\right)+\int_{t_{0}}^{t} \mathrm{e}^{A(t-s)} \dot{f}(s) d s .
$$

Pelo Teorema 1.6.1 (4), $u(t) \in D(A)$ e assim concluímos a prova.

\subsection{Problema de Cauchy Semilinear - Caso Hiperbólico}


Consideremos agora o problema de valor inicial

$$
\begin{aligned}
& \frac{d}{d t} e=A e+f(t, e) \\
& e\left(t_{0}\right)=e_{0} \in E_{0},
\end{aligned}
$$

onde $A$ é o gerador de um $C_{0}$-semigrupo, $f: U \subset \mathbb{R} \times E_{0} \rightarrow E_{0}$ é uma função contínua e $\left(t_{0}, e_{0}\right) \in U$.

\section{Definição 1.7.1.}

a) Uma função contínua e $\left[t_{0}, t_{1}\right) \rightarrow E_{0}$ é uma soluçāo forte de (1.29), em $\left[t_{0}, t_{1}\right)$ se e é $C^{1}$ em $\left(t_{0}, t_{1}\right)$ e tal que $e\left(t_{0}\right)=e_{0}$ e para $t_{0}<t<t_{1},(t, e(t)) \in U, e(t) \in D(A)$ e (1.29) vale.

b) Uma função contínua $e:\left[t_{0}, t_{1}\right) \rightarrow E_{0}$ é uma solução fraca de (1.29) em $\left[t_{0}, t_{1}\right)$ se $e\left(t_{0}\right) \in e_{0}(t, e(t)) \in U, t_{0}<t<t_{1}$ e para todo $e^{*} \in D\left(A^{*}\right), t \mapsto\left\langle e^{*}, e(t)\right)$ é diferenciável $e$

$$
\frac{d^{+}}{d t}\left\langle e^{*}, e(t)\right\rangle=\left\langle A^{*} e^{*}, e(t)\right\rangle+\left\langle e^{*}, f(t, e(t))\right), \quad t_{0}<t<t_{1} .
$$

\section{Teorema 1.7.1.}

1. Toda solução forte de (1.29) é também uma solução fraca de (1.29).

2. Uma solução fraca $e:\left[t_{0}, t_{1}\right) \rightarrow E_{0}$ de (1.29) é também uma solução forte se $e$ somente se é $C^{1}$ em $\left(t_{0}, t_{1}\right)$ se e somente se $e(t) \in D(A)$ com $t \mapsto A e(t)$ contínua em $\left(t_{0}, t_{1}\right)$.

3. Se e $:\left[t_{0}, t_{1}\right) \rightarrow E_{0}$ é uma solução fraca de (1.26), então

$$
e(t)=\mathbf{e}^{A\left(t-t_{0}\right)} e_{0}+\int_{t_{0}}^{t} \mathbf{e}^{A(t-s)} f(s, e(s)) d s, \quad t_{0} \leq t<t_{1} .
$$

4. Se e $:\left[t_{0}, t_{1}\right) \rightarrow E_{0}$ é contínua com $(t, e(t)) \in U, t_{0} \leq t<t_{1}$ e satisfaz (1.31), então $e:\left[t_{0}, t_{1}\right) \rightarrow E_{0}$ é uma solução fraca de (1.29).

Prova: A prova deste teorema segue imediatamente do Teorema 1.6.1 uma vez que

$$
t \mapsto f(t, e(t)):\left[t_{0}, t_{1}\right) \rightarrow E_{0}
$$

é uma função contínua. $\square$ 
Teorema 1.7.2. Suponha que $\left\{\mathrm{e}^{A t} ; t \geq 0\right\}$ é um $C_{0}$-semigrupo, $U \subset \mathbb{R} \times E_{0}$ um aberto e $f: U \rightarrow E_{0}$ contínua e localmente Lipschitz contínua em seu segundo argumento; isto é, dado $\left(t_{0}, x_{0}\right) \in U$ existe $\delta>0$ e $L$ tal que

$$
\left\|f\left(t, e_{1}\right)-f\left(t, e_{2}\right)\right\|_{E_{0}} \leq L\left\|e_{1}-e_{2}\right\|_{E_{0}}
$$

quando $\left|t-t_{0}\right| \leq \delta$ e $\left\|e_{i}-e_{0}\right\|_{E_{0}} \leq \delta, i=1,2$. Então, dado qualquer $\left(t_{0}, e_{0}\right) \in U$ existe $t_{1}>t_{0}$ e uma solução fraca $e:\left[t_{0}, t_{1}\right) \rightarrow E_{0}$ de (1.29). E ainda, qualquer solução fraca $\tilde{e}:\left[t_{0}, \tilde{t}_{1}\right) \rightarrow E_{0}$ é tal que $\tilde{e}(t)=e(t)$ para $t_{0} \leq t<\min \left\{t_{1}, \tilde{t}_{1}\right\}$.

Prova: Como $f: U \rightarrow E_{0}$ é contínua e localmente Lipschitz contínua em seu segundo argumento, existe $\delta>0$ e constantes $L, M$ tais que se $t_{0} \leq t \leq t_{0}+\delta,\left\|e_{i}-e_{0}\right\|_{E_{0}} \leq \delta$, $i=1,2$,

$$
\begin{aligned}
& \left\|f\left(t, e_{1}\right)-f\left(t, e_{2}\right)\right\|_{E_{0}} \leq L\left\|e_{1}-e_{2}\right\|_{E_{0}} \\
& \left\|f\left(t, e_{1}\right)\right\|_{E_{0}} \leq M
\end{aligned}
$$

Dado $t_{1}>t_{0}$ tal que

$$
0<t_{1}-t_{0} \leq \min \left\{\frac{\delta}{2 M M_{0}}, \frac{1}{2 M_{0} L}, \delta, \epsilon\right\}
$$

onde $\left\|\mathrm{e}^{A \tau}\right\|_{L\left(E_{0}\right)} \leq M I_{0}, 0 \leq \tau \leq \delta,\left\|\mathrm{e}^{A \tau} e_{0}-e_{0}\right\|_{E_{0}} \leq \delta / 2$ quando $0 \leq \tau \leq \epsilon$.

Consideremos como $S$ o conjunto das funções contínuas $e:\left[t_{0}, t_{1}\right] \rightarrow E_{0}$ tal que $\left\|e(t)-e_{0}\right\|_{E_{0}} \leq \delta$ para $t_{0} \leq t \leq t_{1}$ e defina $d(e, \tilde{e})=\sup _{t_{0} \leq t \leq t_{1}}\|e(t)-\tilde{e}(t)\|_{E_{0}}$ para $e, \tilde{e} \in S ;$ então $(S, d)$ é um espaço métrico completo. Para $e \in S$ defina $G(e):\left[t_{0}, t_{1}\right] \rightarrow E_{0}$ por

$$
G(e)(t)=\mathrm{e}^{A\left(t-t_{0}\right)} e_{0}+\int_{t_{0}}^{t} \mathrm{e}^{A(t-s)} f(s, e(s)) d s, \quad t_{0} \leq t \leq t_{1}
$$

Temos que $G(S) \subset S$, pois se $e \in S$ e $\quad t_{0} \leq t \leq t_{1}$,

$$
\begin{gathered}
\left\|G(e)(t)-e_{0}\right\|_{E_{0}} \leq\left\|\mathrm{e}^{A\left(t-t_{0}\right)} e_{0}\right\|_{E_{0}}+\int_{t_{0}}^{t}\left\|\mathrm{e}^{A(t-s)}\right\|_{L\left(E_{0}\right)}\|f(s, e(s))\|_{E_{0}} d s \\
\leq \frac{\delta}{2}+M_{0} M\left(t-t_{0}\right) \leq \frac{\delta}{2}+M_{0} M\left(t_{1}-t_{0}\right) \leq \frac{\delta}{2}+M_{0} M \frac{\delta}{2 M M_{0}}=\delta
\end{gathered}
$$

Mostraremos agora que $G: S \rightarrow S$ é uma contração. Para isso, tomemos $e, \tilde{e} \in S$,

$$
\begin{gathered}
d(G(e)-G(\bar{e})) \leq \sup _{t_{0} \leq t \leq t_{1}} \int_{t_{0}}^{t}\left\|\mathrm{e}^{A(t-s)}\right\|_{L\left(E_{0}\right)} \| f(s, e(s))-f\left(s, \tilde{e}(s) \|_{E_{0}} d s\right. \\
\leq \sup _{t_{0} \leq t \leq t_{1}} \int_{t_{0}}^{t} M_{0} L\|e(s)-\tilde{e}(s)\|_{E_{0}} d s \leq \frac{1}{2} d(e, \bar{e})
\end{gathered}
$$


Pelo Teorema da Contração de Banach, temos que $G$ tem um único ponto fixo em $S$, isto é,

$$
e(t)=\mathrm{e}^{A\left(t-t_{0}\right)} e_{0}+\int_{t_{0}}^{t} \mathrm{e}^{A(t-s)} f(s, e(s)) d s .
$$

Isto prova a afirmativa pelo Teorema 1.7 .1 partes (3) e (4) $\cdot \square$

A seguir obtemos resultados sobre extensōes de soluções de (1.29) e a existência de intervalos maximais de definição para soluções de (1.29).

Lema 1.7.1 (Desigualdade de Gronwall). Se $\alpha \in \mathbb{R}, \beta(t) \geq 0$, e $\phi(t)$ são funções reais continuas para $a \leq t \leq b$ que satisfaz

$$
\phi(t) \leq \alpha+\int_{a}^{t} \beta(s) \phi(s) d s, a \leq t \leq b,
$$

então

$$
\phi(t) \leq \mathrm{e}^{\int_{a}^{t} \beta(s) d s} \alpha, a \leq t \leq b
$$

Teorema 1.7.3. Assuma que $A, U$ e f são como no Teorema 1.7.2. Para $\left(t_{0}, e_{0}\right) \in U$ existe uma única solução fraca maximal $e:\left[t_{0}, \tau_{\max }\right) \rightarrow E_{0}$ de (1.29). Suponha que $\tau_{\max }<\infty$, então ou existe $e_{1} \in E_{0}$ tal que $\left(\tau_{\max }, e_{1}\right) \in \partial U$ e e $(t) \rightarrow e_{1}$ quando $t \rightarrow \tau_{\max }$ ou

$$
\limsup _{t \rightarrow \tau_{\max }} \frac{\|f(t, e(t))\|_{E_{0}}}{1+\|e(t)\|_{E_{0}}}=\infty
$$

Prova: Tomemos $\tau_{\max }=\sup \left\{t_{1}\right.$ : existe uma solução de (1.29) definida em $\left.\left[t_{0}, t_{1}\right)\right\}$ e para qualquer $t \in\left[t_{0}, \tau_{\max }\right)$ defina $e(t)=$ \{o valor em $t$ de uma solução fraca $\tilde{e}:\left[t_{0}, t_{1}\right) \rightarrow$ $E_{0}$ de (1.29), $\left.t_{1}>t\right\}$. Ela está bem definida, pois toda solução fraca dá o mesmo valor para $e(t)$. Pelo Teorema 1.7.2 $e:\left[t_{0}, \tau_{\max }\right) \rightarrow E_{0}$ é claramente maximal.

Se $\tau_{\max }<\infty$ e o limite $e_{1}=\lim _{t \rightarrow \tau_{\max }^{-}} e(t)$ existe, então para $\left(\tau_{\max }, e_{1}\right) \in U$ existe uma solução fraca $\tilde{e}:\left[\tau_{\max }, \tau_{\max }+\delta\right] \rightarrow E_{0}$ para algum $\delta>0 \operatorname{com} \tilde{e}\left(\tau_{\max }\right)=e_{1}$. Assim se definirmos $\hat{e}:\left[t_{0}, \tau_{\max }+\delta\right] \rightarrow E_{0}$ por $\hat{e}(t)=e(t), t_{0} \leq t<\tau_{\max }$ e $\hat{e}(t)=\tilde{e}(t)$, $\tau_{\max } \leq t \leq \tau_{\max }+\delta$, temos que $\hat{e}$ é uma solução fraca de (1.29) o que contradiz a definiçāo de $\tau_{\max }$. Com isso, concluímos que, se o limite existe, devemos ter $e_{1} \in \partial U$.

Para finalizar tal prova, mostremos que

$$
\frac{\|f(t, e(t))\|_{E_{0}}}{1+\|e(t)\|_{E_{0}}} \leq B<\infty, \quad t_{0} \leq t<\tau_{\max }
$$


implica que $\lim _{t \rightarrow \tau_{\max }^{-}} e(t)$ existe. Pela desigualdade de Gronwal $\|e(t)\|_{E_{0}}$ é limitada, pois

$$
\|e(t)\| \leq M\left\|e_{0}\right\|_{E_{0}}+\int_{t_{0}}^{t} M B(1+\|e(s)\|) d s,
$$

donde segue que $\|f(t, e(t))\|_{E_{0}} \leq B_{1}, t_{0} \leq t<\tau_{\max }$. Provamos que $\|e(s)-e(r)\|_{E_{0}} \rightarrow 0$ quando $s, t \rightarrow \tau_{\max }^{-}$. Pela limitaçāo exponencial, podemos assumir que $\left\|\mathbf{e}^{A t}\right\| \leq M$ para $0 \leq t \leq \tau_{\max }-t_{0}$. Dado $\epsilon>0$ escolha $0<\epsilon_{1}<\tau_{\max }-t_{0}$ com $\epsilon_{1} \leq \frac{\epsilon}{4 M B_{1}}$. Seja $t^{*}=\tau_{\max }-\epsilon_{1}$ e $0<\delta \leq \epsilon_{1}$ tal que $\left\|\left(\mathrm{e}^{A\left(s-t^{*}\right)}-\mathrm{e}^{A\left(r-t^{*}\right)}\right) e\left(t^{*}\right)\right\|_{E_{0}} \leq \frac{\epsilon}{4}$ se $|s-r| \leq \delta$. Então para $t^{*} \leq \tau_{\max }-\delta \leq s, r<\tau_{\max }$,

$$
e(s)=\mathrm{e}^{A\left(s-t^{*}\right)} e\left(t^{*}\right)+\int_{t^{*}}^{s} \mathrm{e}^{A(s-\theta)} f(\theta, e(\theta)) d \theta,
$$

logo, se $s \leq r$,

$$
\begin{gathered}
\|e(s)-e(r)\|_{E_{0}} \leq\left\|\mathrm{e}^{A\left(s-t^{*}\right)} e\left(t^{*}\right)-\mathrm{e}^{A\left(r-t^{*}\right)} e\left(t^{*}\right)\right\|+\int_{t^{*}}^{s}\left\|\mathrm{e}^{A(s-\theta)}\right\|\|f(\theta, e(\theta))\| d \theta \\
-\int_{t^{*}}^{s}\left\|\mathrm{e}^{A(r-\theta)}\right\|\|f(\theta, e(\theta))\| d \theta-\int_{s}^{r}\left\|\mathrm{e}^{A(r-\theta)}\right\|\|f(\theta, e(\theta))\| d \theta \\
\leq \frac{\epsilon}{4}+2 \int_{t^{*}}^{s} M B_{1} d \theta+\int_{s}^{T} M B_{1} d \theta \leq \epsilon \cdot \square
\end{gathered}
$$

Teorema 1.7.4. Suponha que $A, U$ ef são como no Teorema 1.7.2 e que e $:\left[t_{0}, t_{1}\right) \rightarrow E_{0}$ é uma solução fraca de (1.29). Seja $f_{n}: U \rightarrow E_{0}(n=1,2,3, \cdots)$ tal que $f_{n}(t, e) \rightarrow f(t, e)$ quando $n \rightarrow \propto$ uniformemente para $(t, e)$ em uma vizinhança de cada ponto $(\tau, e(\tau))$, $t_{0} \leq r<t_{1}$. Suponha também que $f_{n}$ satisfaz as condições do Teorema 1.7.2. Se $e_{n}$ : $\left[t_{0}, t_{n}\right) \rightarrow E_{0}$ é uma solução fraca de

$$
\begin{aligned}
& \frac{d}{d t} e_{n}=A e_{n}+f_{n}\left(t, e_{n}(t)\right) \\
& e_{n}\left(t_{0}\right)=e_{n 0},
\end{aligned}
$$

onde $e_{n 0} \rightarrow e_{0}$. então dado $t_{0}<t^{*}<t_{1}$, para $n$ grande, $e_{n}$ está definido em $\left[t_{0}, t^{*}\right]$; isto é, $t_{n}>t^{*} e$

$$
\lim _{n \rightarrow \infty} \sup _{t_{0} \leq t \leq t^{-}}\left\|e_{n}(t)-e(t)\right\|_{E_{0}}=0 .
$$

Prova: Temos que $\left\|\mathrm{e}^{A t}\right\|_{L\left(E_{0}\right)} \leq M$ em $0 \leq t \leq t^{*}-t_{0}$. E pela compacidade de $\{(\tau, e(\tau))$ : $\left.t_{0} \leq \tau \leq t^{*}\right\} \subset L^{*}$, podemos escolher $r>0, L$ e $\epsilon_{n}>0$ tal que, para $y, z$ na bola fechada de raio $r$ em torno de $e(r)$,

$$
\|f(\tau, y)-f(\tau, z)\|_{E_{0}} \leq L\|y-z\|_{E_{0}}
$$


e $\left\|f_{n}(\tau, y)-f(\tau, z)\right\|_{E_{0}} \leq \epsilon_{n}, \epsilon_{n} \rightarrow 0$ quando $n \rightarrow \infty$.

Escolha $n_{0}$ tal que $n \geq n_{0}$ implica

$$
M\left[\left\|e_{n}\left(t_{0}\right)-e\left(t_{0}\right)\right\|_{E_{0}}+\epsilon_{n}\left(t^{*}-t_{0}\right)\right] \mathrm{e}^{M L\left(t^{*}-t_{0}\right)}<r .
$$

Donde segue que, $\left\|\mathrm{e}^{A\left(t-t_{0}\right)}\left(e_{n}\left(t_{0}\right)-e\left(t_{0}\right)\right)\right\|<r$ e $\left\|e_{n}(t)-e(t)\right\|_{E_{0}}<r$ para $t$ próximo a $t_{0}$. Digamos que $n \geq n_{0}$ e $\left\|e_{n}(s)-e(s)\right\|_{E_{0}} \leq r$ para $t_{0} \leq s<t \leq t^{*}$; então

$$
\begin{aligned}
\left\|e_{n}(t)-e(t)\right\|_{E_{0}} & \leq\left\|\mathrm{e}^{A\left(t-t_{0}\right)}\left(e_{n}\left(t_{0}\right)-e\left(t_{0}\right)\right)+\int_{t_{0}}^{t} \mathrm{e}^{A(t-s)}\left(f_{n}\left(s, e_{n}(s)\right)-f\left(s, e_{n}(s)\right)\right) d s\right\|_{E_{0}} \\
& +\left\|\int_{t_{0}}^{t} \mathrm{e}^{A(t-s)}\left(f\left(s, e_{n}(s)\right)-f(s, e(s))\right) d s\right\|_{E_{0}} \\
& \leq M\left\|e_{n}\left(t_{0}\right)-e\left(t_{0}\right)\right\|_{E_{0}}+M \epsilon_{n}\left(t-t_{0}\right)+\int_{t_{0}}^{t} M L\left\|e_{n}(s)-e(s)\right\|_{E_{0}} d s
\end{aligned}
$$

Pela desigualdade de Gronwall temos que $\left\|e_{n}(t)-e(t)\right\|_{E_{0}}<r$ (se $n \geq n_{0}$ ) logo a desigualdade vale para todo $t_{0} \leq t \leq t^{*} \mathrm{e}$

$$
\left\|e_{n}(t)-e(t)\right\|_{E_{0}} \leq M\left[\left\|e_{n}\left(t_{0}\right)-e\left(t_{0}\right)\right\|_{E_{0}}+\epsilon_{n}\left(t-t_{0}\right)\right] \mathrm{e}^{M L\left(t-t_{0}\right)} \rightarrow 0
$$

quando $n \rightarrow \infty$.

\subsection{O Problema de Cauchy Semilinear - Caso Parabólico}

Nesta seção consideramos problemas de Cauchy da forma

$$
\begin{aligned}
& \frac{d}{d t} e=-A e+f(t, e), \\
& e\left(t_{0}\right)=e_{0}
\end{aligned}
$$

onde $A: D(A) \subset E_{0} \rightarrow E_{0}$ é um operador setorial positivo e $f: U \rightarrow E_{0}$ é uma função contínua e $U \subset \mathbb{R} \times E^{\alpha}$ é um aberto.

Note que, como o operador $A$ é de tipo posivivo podemos definir as suas potências fracionárias $A^{\alpha}, \alpha \in \mathbb{R}$.

Teorema 1.8.1. Seja $E^{\alpha}$ o domínio de $A^{\alpha}$ dotado com a norma do gráfico, entâo:

(i) $E^{a}$ é um espaço de Banach; 
(ii) A restrição de $\left\{\mathrm{e}^{-A t} ; t \geq 0\right\}$ a $E^{\alpha}$ é um $C_{0}$-semigrupo em $E^{\alpha}$.

Como $-A$ é gerador de um $C_{0}$-semigrupo, os resultados obtidos para o caso hiperbólico também se aplicam a (1.34). Assim, obtemos teoremas de existência e regularidade apropriados para o caso parabólico. O que faremos aqui é mostrar que, toda solução de (1.34) com dados iniciais em algum $E^{\alpha}, \alpha<1$ são soluções fortes.

Definição 1.8.1. Seja $A: D(A) \subset E_{0} \rightarrow E_{0}$ um operador setorial e escolha $a \geq 0$ (se necessário) tal que $(-\infty, 0] \subset \rho(A+a)$. Considere $0 \leq \alpha \leq 1$ e $E^{\alpha}=D\left((A+a)^{\alpha}\right)$ com a norma do gráfico $\|\cdot\|_{\alpha}=\left\|(A+a)^{\alpha} \cdot\right\|_{E_{0}}$.

Uma função contínua $e:\left[t_{0}, t_{1}\right) \rightarrow E_{0}$ é uma solução de (1.34) em $\left[t_{0}, t_{1}\right)$, se e é diferenciável em $\left(t_{0}, t_{1}\right) e(t, e(t)) \in U, t_{0} \leq t<t_{1}, e(t) \in D(A), t_{0}<t<t_{1}$, $t \mapsto A e(t):\left(t_{0}, t_{1}\right) \rightarrow E_{0}$ é contínua e (1.34) está satisfeita.

Através do teorema a seguir, temos que o conceito de solução fraca não se faz necessário para (1.34).

Teorema 1.8.2. Assuma que $E_{0}, A, \alpha, U$ e f são como na Definição (1.8.1) e suponha que $f$ é localmente Hölder contínua; isto é, qualquer ponto de $U$ tem uma vizinhança $V \subset U$ e constantes $K, \theta>0$, tais que quando $\left(\tau_{1}, e_{1}\right)$ e $\left(\tau_{2}, e_{2}\right)$ estão em $V$

$$
\left\|f\left(\tau_{1}, e_{1}\right)-f\left(\tau_{2}, e_{2}\right)\right\|_{E_{0}} \leq K\left(\left|\tau_{1}-\tau_{2}\right|^{\theta}+\left\|e_{1}-e_{2}\right\|_{E^{\alpha}}^{\theta}\right) .
$$

Se e $:\left[t_{0}, t_{1}\right] \rightarrow E^{\alpha}$ é contínua, $(t, e(t)) \in U, t_{0} \leq t \leq t_{1}, e$

$$
e(t)=\mathrm{e}^{-A\left(t-t_{0}\right)} e\left(t_{0}\right)+\int_{t_{0}}^{t} \mathrm{e}^{-A(t-s)} f(s, e(s)) d s, \quad t_{0} \leq t \leq t_{1},
$$

então e(t) é uma solução forte de (1.34).

Prova: Primeiramente mostraremos que $e:\left(t_{0}, t_{1}\right] \rightarrow E^{\alpha}$ é Hölder contínua. Como $\left\{(t, e(t)): t_{0} \leq t \leq t_{1}\right\}$ é um subconjunto compacto de $U$, existe $B$ tal que

$$
\sup _{t_{0} \leq t \leq t_{1}} \| f\left(t, e(t) \|_{E_{0}} \leq B .\right.
$$

Então, para $t_{0}<t \leq t+h \leq t_{1}$,

$$
\begin{aligned}
e(t+h)-e(t) & =\left(\mathrm{e}^{-A h}-I\right)\left[\mathrm{e}^{-A\left(t-t_{0}\right)} e\left(t_{0}\right)+\int_{t_{0}}^{t} \mathrm{e}^{-A(t-s)} f(s, e(s)) d s\right] \\
& +\int_{t}^{t+h} \mathrm{e}^{-A(t+h-s)} f(s, e(s)) d s
\end{aligned}
$$


logo, se $0<\theta<1-\alpha$, e $M$ é dada pelo Teorema 1.5.10

$$
\begin{aligned}
\|e(t+h)-e(t)\|_{\alpha} & \leq M h^{\theta}\left(t-t_{0}\right)^{-\theta}\left\|e\left(t_{0}\right)\right\|_{\alpha}+\int_{t_{0}}^{t} M h^{\theta}(t-s)^{-\alpha-\theta} B d s \\
& +\int_{t}^{t+h} M(t+h-s)^{-\alpha} B d s \\
& =\mathrm{O}\left(h^{\theta}\left(t-t_{0}\right)^{-\theta}\right) .
\end{aligned}
$$

Segue que $t \mapsto f(t, e(t)) \equiv g(t)$ é contínua em $\left[t_{0}, t_{1}\right]$ e satisfaz uma condição de Hölder

$$
\|g(t+h)-g(t)\|_{E_{0}} \leq K\left(t-t_{0}\right)^{-\delta} h^{\delta}, \quad t_{0}<t \leq t+h \leq t_{1},
$$

para alguma escolha de $K, \delta>0$ (e $0<\delta<1-\alpha$, sem perda de generalidade). É suficiente provar que

$$
G(t)=\int_{t_{0}}^{t} \mathrm{e}^{-A(t-s)} g(s) d s
$$

toma valores em $D(A)$ com $t \rightarrow A G(t)$ contínua em $\left(t_{0}, t_{1}\right]$, e portanto mostraremos que $h^{-1}\left(\mathrm{e}^{-A h}-I\right) G(t)$ converge quando $h \rightarrow 0^{+}$, uniformemente para $t_{0}^{*} \leq t \leq t_{1}$, qualquer que seja $t_{0}^{*}>t_{0}$. Agora

$$
\begin{aligned}
h^{-1}\left(\mathrm{e}^{-A h}-I\right) G(t) & =\int_{t_{0}}^{t} h^{-1}\left(\mathrm{e}^{-A h}-I\right) \mathrm{e}^{-A(t-s)}(g(s)-g(t)) d s \\
& +h^{-1} \int_{t_{0}}^{t_{0}+h} \mathrm{e}^{-A(t+h-s)} g(t) d s-h^{-1} \int_{t-h}^{t} \mathrm{e}^{-A(t-s)} g(t) d s
\end{aligned}
$$

e os dois últimos termos são claramente convergentes uniformemente em $t$. Para o outro termo, primeiramente note que

$$
\int_{t_{0}}^{t}\left\|A \mathrm{e}^{-A(t-s)}\right\|_{L\left(E_{0}\right)}\|g(t)-g(s)\|_{E_{0}} d s<\infty
$$

da condição de Hölder, e

$$
\begin{aligned}
&\left\|h^{-1} \int_{t_{0}}^{t}\left(\mathrm{e}^{-A h}-I+h A\right) \mathrm{e}^{-A(t-s)}(g(s)-g(t)) d s\right\|_{E_{0}} \\
&=\left\|\int_{t_{0}}^{t} h^{-1} \int_{0}^{h}\left(I-\mathrm{e}^{-A \sigma}\right) d \sigma A \mathrm{e}^{-A(t-s)}(g(s)-g(t)) d s\right\|_{E_{0}} \\
& \leq \int_{t_{0}}^{t} M h^{\epsilon}(t-s)^{-1-\epsilon} K\left(s-t_{0}\right)^{-\delta}(t-s)^{\delta} d s \stackrel{h \rightarrow 0^{+}}{\longrightarrow} 0, \quad 0<\epsilon<\delta
\end{aligned}
$$

uniformemente para $t_{0}^{*} \leq t \leq t_{1}$.

Portanto, $h^{-1}\left(\mathrm{e}^{-A h}-I\right) G(t) \rightarrow-\int_{t_{0}}^{t} A \mathrm{e}^{-A(t-s)}(g(s)-g(t)) d s+\mathrm{e}^{-A\left(t-t_{0}\right)} g(t)-g(t)$ quando $h \rightarrow 0^{+}$uniformemente em $\left[t_{0}^{*}, t_{1}\right]$, e a prova está completa. $\square$

As integrais impróprias, que aparecem nas estimativas obtidas através da fórmula da variaçāo das constantes no caso parabólico, tornam necessárias a obtenção de uma 
desigualdade de Gronwal onde as funções conhecidas possuem singularidades. Uma dessas desigualdades é obtida no lema a seguir (veja [6]).

Lema 1.8.1. Assuma que $a, b \geq 0,0 \leq \alpha, \beta<1$ e $u:[0, T] \rightarrow \mathbb{R}$ é integrável com

$$
0 \leq u(t) \leq a t^{-\alpha}+b \int_{0}^{t}(t-s)^{-\beta} u(s) d s
$$

quase sempre em $(0, T)$. Então, existe uma constante $K$ dependendo somente de $b, \beta, T$ tal que

$$
u(t) \leq \frac{K}{1-\alpha} a t^{-\alpha}
$$

quase sempre em $(0, T)$.

Prova: Defina a transformação $B: \phi \mapsto\left\{b \int_{0}^{t}(t-s)^{-\beta} \phi(s) d s, 0 \leq t \leq T\right\}$. Então, a desigualdade (1.35) pode ser escrita na forma

$$
0 \leq u(t) \leq u_{0}(t)+B u(t)
$$

onde $u_{0}(t)=a t^{-\alpha}$. É claro que $B$ preserva ordem e portanto

$$
0 \leq u(t) \leq u_{0}(t)+B u(t) \leq u_{0}(t)+B u_{0}(t)+B^{2} u(t) \leq \cdots \leq \sum_{k=0}^{m-1} B^{k} u_{0}(t)+B^{m} u(t)
$$

para $m=1,2,3, \cdots$. Vamos mostrar que a série $\sum_{n=0}^{\infty} B^{n} u_{0}(t)$ converge e que $B^{n} u(t) \rightarrow 0$ quando $n \rightarrow \infty$ e depois obter a estimativa

$$
\sum_{n=0}^{\infty} B^{n} u_{0}(t) \leq \frac{K}{1-\alpha} a t^{-\alpha}
$$

Suponha, por indução, que

$$
B^{n-1} \phi(t)=\int_{0}^{t} \frac{(b \Gamma(1-\beta))^{n-1}}{\Gamma((n-1)(1-\beta))}(t-s)^{(n-1)(1-\beta)-1} \phi(s) d s \quad n \geq 2
$$


então obtemos

$$
\begin{aligned}
B\left(B^{n-1} \phi\right)(t) & =b \int_{0}^{t}(t-s)^{-\beta}\left[\int_{0}^{s} \frac{(b \Gamma(1-\beta))^{n-1}}{\Gamma((n-1)(1-\beta))}(s-\theta)^{(n-1)(1-\beta)-1} \phi(\theta) d \theta\right] d s \\
& =\frac{b^{n} \Gamma(1-\beta)^{n-1}}{\Gamma((n-1)(1-\beta))} \int_{0}^{t} \phi(\theta)\left[\int_{\theta}^{t}(t-s)^{-\beta}(s-\theta)^{(n-1)(1-\beta)-1} \phi(\theta) d s\right] d \theta \\
& =\frac{b^{n} \Gamma(1-\beta)^{n-1}}{\Gamma((n-1)(1-\beta))} \int_{0}^{t} \phi(\theta)\left[\int_{0}^{t-\theta}(t-\theta-u)^{-\beta} u^{(n-1)(1-\beta)-1} d u\right] d \theta \\
& =\frac{b^{n} \Gamma(1-\beta)^{n-1}}{\Gamma((n-1)(1-\beta))} \int_{0}^{t} \phi(\theta)(t-\theta)^{n(1-\beta)-1} \int_{0}^{1}(1-z)^{-\beta} z^{(n-1)(1-\beta)-1} d z d \theta \\
& =\frac{b^{n} \Gamma(1-\beta)^{n-1}}{\Gamma((n-1)(1-\beta))} \mathbf{B}(1-\beta,(n-1)(1-\beta)) \int_{0}^{t} \phi(\theta)(t-\theta)^{n(1-\beta)-1} d \theta \\
& =\frac{b^{n} \Gamma(1-\beta)^{n}}{\Gamma(n(1-\beta))} \int_{0}^{t} \phi(\theta)(t-\theta)^{n(1-\beta)-1} d \theta \\
& =B^{n} \phi(t)
\end{aligned}
$$

onde utilizamos que $\mathbf{B}(x, y)=\int_{0}^{1} z^{x-1}(1-z)^{y-1} d z=\frac{\Gamma(x) \Gamma(y)}{\Gamma(x+y)}$.

Agora vamos estudar a convergência da série

$$
S_{\beta}(z)=\sum_{n=0}^{\infty} \frac{z^{n}}{\Gamma(n(1-\beta))}
$$

note que se $a_{n}=\Gamma(n(1-\beta))^{-1}$ temos que

$$
\begin{aligned}
\frac{a_{n+1}}{a_{n}} & =\frac{\Gamma(n(1-\beta))}{\Gamma((n+1)(1-\beta))}=\frac{\mathbf{B}((1-\beta, n(1-\beta))}{\Gamma(1-\beta)} \\
& =\frac{1}{\Gamma(1-\beta)} \int_{0}^{1} t^{-\beta}(1-t)^{n(1-\beta)-1} d t \rightarrow 0
\end{aligned}
$$

quando $n \rightarrow \infty$ pelo Teorema da Convergência Dominada de Lebesgue.

Isto mostra que $B^{n} \phi(t) \rightarrow 0$ quando $n \rightarrow \infty$ e segue que

$$
u(t) \leq \sum_{n=0}^{\infty}\left(B^{n} u_{0}\right)(t)
$$


Resta encontrar uma estimativa para a série acima. Note que

$$
\begin{aligned}
B^{n} u_{0}(t) & =a \frac{b^{n} \Gamma(1-\beta)^{n}}{\Gamma(n(1-\beta))} \int_{0}^{t}(t-s)^{n(1-\beta)-1} s^{-\alpha} d s \\
& =a t^{-\alpha} \frac{z^{n(1-\beta)}}{\Gamma(n(1-\beta))} \mathbf{B}(1-\alpha, n(1-\beta)) \\
& \leq \frac{a}{1-\alpha} t^{-\alpha} \frac{z^{n(1-\beta)}}{\Gamma(n(1-\beta))}
\end{aligned}
$$

onde $z=t(b \Gamma(1-\beta))^{1 /(1-\beta)}$. Defina, para $z \geq 0$,

$$
E_{\beta}(z):=\sum_{n=0}^{\infty} \frac{z^{n(1-\beta)}}{\Gamma(n(1-\beta)+1)} .
$$

A convergência da série acima pode ser garantida como antes. Pode ser provado (veja [5], página 38) que $E_{\beta}(z) \leq c \mathbf{e}^{z}$.

Escolha um número natural $k$ tal que $k(1-\beta)>1$, então

$$
\begin{aligned}
S_{\beta}(z) & \leq \sum_{j=0}^{k-1} \frac{z^{j(1-\beta)}}{\Gamma(j(1-\beta))}+\frac{z^{k(1-\beta)}}{\Gamma(k(1-\beta))} \sum_{n=0}^{\infty} \frac{z^{n(1-\beta)}}{\Gamma(n(1-\beta)+1)} \\
& \leq \sum_{j=0}^{k-1} \frac{z^{j(1-\beta)}}{\Gamma(j(1-\beta))}+c \frac{z^{k(1-\beta)}}{\Gamma(k(1-\beta))} \mathbf{e}^{z}
\end{aligned}
$$

para algum $c>0$. Disto obtemos que

$$
u(t) \leq \frac{a}{1-\alpha} t^{-\alpha}\left[\sum_{j=0}^{k-1} \frac{t^{j(1-\beta)}(b \Gamma(1-\beta))^{j}}{\Gamma(j(1-\beta))}+c(b \Gamma(1-\beta))^{k} \frac{t^{k(1-\beta)}}{\Gamma(k(1-\beta))} \mathrm{e}^{t(b \Gamma(1-\beta))^{1 /(1-\beta)}}\right]
$$

e o resultado segue. Observe que de fato provamos mais do que está enunciado. A prova do resultado enunciado somente utiliza a continuidade de $S_{\beta}$, não fazendo uso dos resultados em $([5]) \cdot$.

Teorema 1.8.3. Assuma que $E_{0}$ é um espaço de Banach, $A$ é um operador setorial em $E_{0}, 0 \leq \alpha<1, E^{\alpha}$ é definido como anteriormente e $U$ é um subconjunto aberto de $\mathbb{R} \times E^{\alpha}$. Suponha que $f: U \rightarrow E_{0}$ é localmente Hölder contínua e localmente Lipschitz contínua em seu segundo argumento; ou seja, em uma vizinhança de qualquer ponto de $U$ temos

$$
\left\|f\left(t, e_{1}\right)-f\left(s, e_{2}\right)\right\|_{E_{0}} \leq C\left(|t-s|^{\theta}+\left\|e_{1}-e_{2}\right\|_{E^{\alpha}}\right)
$$


para algum $\theta>0$. Dado qualquer $\left(t_{0}, e_{0}\right) \in U$, existe uma única solução $e:\left[t_{0}, t_{1}\right) \rightarrow E_{0}$ de (1.34) definida em um intervalo maximal. Adicionalmente, se $e_{0} \in D(A)$ a derivada é contínua quando $t \rightarrow t_{0}^{+}$. Se $t_{1}<\infty$ então, quando $t \rightarrow t_{1}$ ou $(t, e(t))$ tende para algum ponto de $\partial U$ ou

$$
\frac{\|f(t, e(t))\|_{E_{0}}}{1+\|e(t)\|_{E^{\alpha}}}
$$

é ilimitada (ou ambos).

Prova: Como no Teorema 1.7.2 e no Teorema 1.7.3, primeiramente provamos a existencia local obtendo que a transformação

$$
G(e)(t)=\mathrm{e}^{-A\left(t-t_{0}\right)} e_{0}+\int_{t_{0}}^{t} \mathrm{e}^{A(t-s)} f(s, e(s)) d s, \quad t_{0} \leq t \leq t_{0}+T
$$

é uma contração em uma bola fechada $B_{r} \subset C\left(\left[t_{0}, t_{0}+T\right], E^{\alpha}\right)$, quando $e:\left[t_{0}, t_{0}+T\right] \rightarrow E^{\alpha}$ é contínua com $\sup _{t_{0} \leq t \leq t_{0}+T}\left\|e(t)-e_{0}\right\|_{E^{\alpha}} \leq r$; isto é, $e(\cdot) \in B_{r}$. Escolhemos $r, T>$ 0 pequenos de forma que para $t \in\left[t_{0}, t_{0}+T\right]$ e $\left\|e_{i}-e_{0}\right\|_{E_{\alpha}} \leq r$, temos $\left(t, e_{i}\right) \in U$, $\left\|f\left(t, e_{i}\right)\right\|_{E_{0}} \leq B, i=1.2 \mathrm{e}$

$$
\begin{aligned}
& \left\|f\left(t, e_{1}\right)-f\left(t, e_{2}\right)\right\|_{E_{0}} \leq L\left\|e_{1}-e_{2}\right\|_{E^{\alpha}}, \quad 0 \leq s \leq T \\
& \left\|\mathrm{e}^{-A s} z\right\|_{E^{\alpha}} \leq M\|z\|_{E^{\alpha}}, \quad 0 \leq s \leq T \\
& \left\|\mathrm{e}^{-A s} z\right\|_{E^{\alpha}} \leq M s^{-\alpha}\|z\|_{E_{0}}, \quad 0 \leq s \leq T \\
& \left\|\mathrm{e}^{-A s} e_{0}-e_{0}\right\|_{E^{\alpha}} \leq r / 2, \quad 0 \leq s \leq T \\
& \frac{M B T^{1-\alpha}}{1-\alpha} \leq r / 2, \\
& \frac{M L T^{1-\alpha}}{1-\alpha} \leq 1 / 2,
\end{aligned}
$$

onde adotamos o procedimento seguinte: primeiramente escolha $r$ e um $T_{0}$ para encontrar $B, L, M$ : então escolha um $T$ menor que $T_{0}$ para ter as condiçãoes acima satisfeitas.

Com estas escolhas. $G$ leva $B_{r}$ nela mesma e é uma contração na norma de $C\left(\left[t_{0}, t_{0}+\right.\right.$ $\left.T], E^{\alpha}\right)$, logo existe um ponto fixo. Segue do Teorema 1.8.2, ela é uma solução de (1.34). 
Para provar a unicidade, suponha que $e, \tilde{e}$ são duas soluções de (1.34), ambas definidas em $\left[t_{0}, t_{2}\right]$. Se elas coincidem em $\left[t_{0}, t_{3}\right]$ mas não coincidem em um intervalo maior, podemos substituir $t_{0}$ por $t_{3}$. Logo, assuma que $e(t) \neq \tilde{e}(t)$ para $t$ arbitrariamente próximo de $t_{0}, t>t_{0}$. Como $e, \tilde{e}$ são contínuas, podemos assumir que isto ocorre para $t \in\left[t_{0}, t_{0}+T\right]$ e $\left\|e(t)-e_{0}\right\|_{E^{\alpha}} \leq r,\left\|\tilde{e}(t)-e_{0}\right\|_{E^{\alpha}} \leq r$. Mas as restrições de $e, \tilde{e}$ a $\left[t_{0}, t_{0}+T\right]$ são pontos fixos de $G$, contradizendo a unicidade dos pontos fixos.

Estendemos as soluções locais ao intervalo maximal de existência como no Teorema 1.7.3 e completamos a prova aplicando a desigualdade de Gronwall do Lema 1.8.1.

O teorema a seguir nos dá informações adicionais sobre a regularidade das soluções de (1.34).

Teorema 1.8.4. Assuma que $E_{0}, A, \alpha, U, f$ são como no Teorema 1.8 .3 e assuma que $t \mapsto f(t, e)$ satisfaz uma condição de Hölder local com expoente $\theta \leq 1$, próximo de cada $(t, e) \in U$. Então, qualquer solução $e:\left[t_{0}, t_{1}\right] \rightarrow E^{\alpha}$ de (1.34) é tal que $t \mapsto \frac{d}{d t} e(t) \in E^{\gamma}$ é localmente Hölder contínua em $\left(t_{0}, t_{1}\right)$ para qualquer $0 \leq \gamma<\theta$ e

$$
\left\|\frac{d}{d t} e(t)\right\|_{E^{\gamma}}=\mathrm{O}\left(\left(t-t_{0}\right)^{\alpha-\gamma-1}\right) \text { quando } t \rightarrow t_{0} .
$$

Prova: Como $\left[t_{0}, t_{1}\right]$ é um intervalo compacto, para algum $h_{0}>0$, se $t_{0} \leq t \leq t+h \leq t_{1}$, $0 \leq h \leq h_{0},\|f(t, e(t))\|_{E_{0}} \leq B$ e

$$
\|f(t+h, e(t+h))-f(t, e(t))\|_{E_{0}} \leq L\left(h^{\theta}+\|e(t+h)-e(t)\|_{E^{\alpha}}\right),
$$

para alguma escolha de $B, L$. Se $M$ é tal que

$$
\begin{aligned}
& \left\|\mathrm{e}^{-A s} z\right\|_{E^{\alpha}} \leq M\|z\|_{E^{\alpha}}, \quad 0 \leq s \leq t_{1}-t_{0} \\
& \left\|\mathrm{e}^{-A s} z\right\|_{E^{\alpha}} \leq M s^{-\alpha}\|z\|_{E_{0}}, \quad 0 \leq s \leq t_{1}-t_{0} \\
& \left\|\mathrm{e}^{-A s} z-z\right\|_{E_{0}} \leq M s^{\theta}\|z\|_{E^{\theta}}, \quad 0 \leq s \leq t_{1}-t_{0}
\end{aligned}
$$

temos

$$
\begin{aligned}
\|e(t+h)-e(t)\|_{E^{\alpha}} & \leq M^{2} h^{\theta}\left(t-t_{0}\right)^{-\theta}\left\|e\left(t_{0}\right)\right\|_{E^{\alpha}}+\int_{t_{0}}^{t_{0}+h} M(t+h-s)^{-\alpha} B d s \\
& +\int_{t_{0}}^{t} M(t-s)^{-\alpha} L\left[h^{\theta}+\|e(s+h)-e(s)\|_{E^{\alpha}}\right] d s \\
& \leq C h^{\theta}\left[\left(t-t_{0}\right)^{-\theta}+\left(t-t_{0}\right)^{-\alpha}\right] \\
& \left.+M L \int_{t_{0}}^{t}(t-s)^{-\alpha}\|e(s+h)-e(s)\|_{E^{\alpha}}\right] d s .
\end{aligned}
$$


Em seguida, usamos o Lema 1.8.1 para concluir $\|e(t+h)-e(t)\|_{E^{\alpha}}=\mathrm{O}\left(h^{\theta}\left[\left(t-t_{0}\right)^{-\theta}+\right.\right.$ $\left.\left.\left(t-t_{0}\right)^{-\alpha}\right]\right)$ e portanto $g(t):=f(t, e(t))$ satisfaz

$$
\|g(t+h)-g(t)\|_{E_{0}} \leq K h^{\theta}\left[\left(t-t_{0}\right)^{-\theta}+\left(t-t_{0}\right)^{-\alpha}\right], \quad\|g(t)\|_{E_{0}} \leq B
$$

para $t_{0}<t \leq t+h \leq t_{1}, 0 \leq h \leq h_{0}$.

Mostramos na prova do Teorema 1.8.2 que

$$
-A \int_{t_{0}}^{t} \mathrm{e}^{-A(t-s)} g(s) d s=-\int_{t_{0}}^{t} A \mathrm{e}^{-A(t-s)}(g(s)-g(t)) d s+\mathrm{e}^{-A\left(t-t_{0}\right)} g(t)-g(t)
$$

e segue que

$$
\frac{d}{d t} e(t)=-A e(t)+g(t)=-A \mathrm{e}^{-A\left(t-t_{0}\right)} e\left(t_{0}\right)+\mathrm{e}^{-A\left(t-t_{0}\right)} g(t)+G(t)
$$

onde $G(t)=\int_{t_{0}}^{t} A \mathrm{e}^{-A(t-s)}(g(s)-g(t)) d s$. Somente o último termo $G$ apresenta dificuldades. Mostraremos que $\|G(t+h)-G(t)\|_{E^{\gamma}}=\mathrm{O}\left(h^{\theta-\gamma}\right)$ quando $h \rightarrow 0^{+}$, sempre que $0 \leq \gamma<\theta$ e $t_{0}<t \leq t_{1}$.

Agora,

$$
\begin{aligned}
G(t+h)-G(t) & =\int_{t_{0}}^{t_{0}+h} A \mathrm{e}^{-A(t+h-s)}(g(s)-g(t+h)) d s \\
& +\int_{t_{0}}^{t} A \mathrm{e}^{-A(t-s)}(g(s+h)-g(t+h)-g(s)+g(t)) d s
\end{aligned}
$$

$\mathrm{e}\|g(t+h)-g(s+h)-g(t)+g(s)\|_{E_{0}} \leq 2 K\left[\left(s-t_{0}\right)^{-\theta}+\left(s-t_{0}\right)^{-\alpha}\right] \min \left\{(t-s)^{\theta}, h^{\theta}\right\} \log 0$

$$
\begin{aligned}
\|G(t+h)-G(t)\|_{E^{\gamma}} & \leq \mathrm{O}(h)+\mathrm{O}\left(\int_{t_{0}}^{t-h} h^{\theta}(t-s)^{-1-\gamma}\left[\left(s-t_{0}\right)^{-\theta}+\left(s-t_{0}\right)^{-\alpha}\right] d s\right) \\
& +\mathrm{O}\left(\int_{t-h}^{t}(t-s)^{-1-\gamma+\theta}\left[\left(s-t_{0}\right)^{-\theta}+\left(s-t_{0}\right)^{-\alpha}\right] d s\right) \\
& =\mathrm{O}\left(h^{\theta-\gamma}\right), \quad \text { quando } h \rightarrow 0^{+} \cdot \square
\end{aligned}
$$

No caso particular de problemas autonomos temos o seguinte resultado:

Corolário 1.8.1. Assuma que $E_{0}, A, \alpha, U, f$ são como no Teorema 1.8 .3 e assuma que $f$ é independente de t. Então, qualquer solução $e:\left[t_{0}, t_{1}\right] \rightarrow E^{\alpha}$ de (1.34) é tal que $t \mapsto \frac{d}{d t} e(t) \in E^{\gamma}$ é localmente Hölder contínua em $\left(t_{0}, t_{1}\right)$ para qualquer $0 \leq \gamma<1$ e $\left\|\frac{d}{d t} e(t)\right\|_{E^{\gamma}}=\mathrm{O}\left(\left(t-t_{0}\right)^{\alpha-\gamma-1}\right)$ quando $t \rightarrow t_{0}$. 


\subsection{O Espaço de Sobolev $W^{1, p}$}

Seja $I=(a, b) \subset \mathbb{R}$ não necessariamente limitado e consideremos $p \in \mathbb{R}$ tal que $1 \leq p \leq \infty$.

Definição 1.9.1. $O$ espaço de Sobolev $W^{1, p}$ é definido por:

$$
W^{1, p}=\left\{u \in L^{p}(I): \exists g \in L^{P}(I) \text { tal que } \int_{I} u \phi^{\prime}=-\int_{I} g \phi \forall \phi \in C_{c}^{\infty}\right\}
$$

onde $C_{c}^{\infty}(I)$ denota o conjunto das funções infinitamente deriváveis com suporte compacto contido em $I$ e para $u \in W^{1, p}$ denotaremos $g$ por $u^{\prime}$. Quando $p=2$, escreveremos $W^{1, p}(I)=H^{1}(I)$.

O espaço de Sobolev $W^{1, p}$ está dotado da norma:

$$
\|u\|_{W^{1, p}}=\|u\|_{L^{p}}+\left\|u^{\prime}\right\|_{L^{p}}
$$

onde

$$
\|u\|_{L^{p}}=\left(\int_{I}|u(x)|^{p} d x\right)^{1 / p}, 1 \leq p<\infty
$$

e

$$
\|u\|_{L^{\infty}}=\inf \{k \geq 0:|u(x)| \leq k \text { quase sempre em } I\} .
$$

E sobre $H^{1}$ definimos o produto interno dado por:

$$
(u, v)_{H^{1}}=(u, v)_{L^{2}}+\left(u^{\prime}, v^{\prime}\right)_{L^{2}}
$$

e a norma associada é

$$
\|u\|_{H^{1}}=\left(\|u\|_{L^{2}}^{2}+\left\|u^{\prime}\right\|_{L^{2}}^{2}\right)^{1 / 2}
$$

que é equivalente a $\|u\|_{W^{1,2}}$

Teorema 1.9.1. $O$ espaço $W^{1, p}$ é um espaço de Banach.

O teorema a seguir mostra que toda função $u \in W^{1, p}$ admite um representante contínuo.

Teorema 1.9.2. Seja $u \in W^{1, p}$; então existe uma função $\tilde{u} \in C(\bar{I})$ tal que

$$
u=\tilde{u} \text { quase sempre em I }
$$

$e$

$$
\tilde{u}(x)-\tilde{u}(y)=\int_{y}^{x} u^{\prime}(t) d t \forall x, y \in I
$$


Teorema 1.9.3. Seja $u \in L^{P}$ com $1<p \leq \infty$. As seguintes afirmações são equivalentes:

(i) $u \in W^{1, p}$

(ii) Existe uma constante $C$ tal que

$$
\left|\int_{I} u \varphi^{\prime}\right| \leq C\|\varphi\|_{L^{p^{\prime}}(I)} \forall \varphi \in C_{c}^{\infty}(I)
$$

(iii) Existe uma constante $C$ tal que para todo aberto $\omega \subset \subset$ I e todo $h \in \mathbb{R}$ com $|h|<$ $\operatorname{dist}\left(\omega, I^{C}\right)$ se verifica

$$
\left\|\tau_{h} u-u\right\|_{L^{p}(\omega)} \leq C|h|
$$

Algumas operações fundamentais da Análise têm sentido apenas para funções definidas em todo IR. Através do resultado a seguir podemos prolongar uma função $u \in W^{1, p}(I)$ a uma função $u \in W^{1, p}(\mathbb{R})$.

Teorema 1.9.4. (Operador Prolongação) Seja $1 \leq p \leq \infty$. Existe um operador prolongação $P: W^{1, p}(I) \rightarrow W^{1, p}(\mathbb{R})$ linear e contínuo tal que

(i) $P u_{\mid I}=u \quad \forall u \in W^{1, p}(I)$

(ii) $\|P u\|_{L^{p(\mathbb{R})}} \leq C\|u\|_{L^{p}(I)} \quad \forall u \in W^{1, p}(I)$

(iii) $\|P u\|_{W^{1, p(\mathbb{R})} \leq C\|u\|_{W^{1, p}(I)}} \forall u \in W^{1, p}(I)$

Teorema 1.9.5. (Densidade) Seja $u \in W^{1, p}$ com $1 \leq p<\infty$. Então existe uma seqüência $\left(u_{n}\right)$ em $C_{c}^{\infty}(\mathbb{R})$ tal que $u_{n ; I} \rightarrow u \in W^{1, p}(I)$.

Teorema 1.9.6. Existe uma constante $C$ (dependendo somente de $|I| \leq \infty$ ) tal que

$$
\|u\|_{L^{\infty}(I)} \leq C\|u\|_{W^{1, p(I)}} \quad \forall u \in W^{1, p}(I) \quad 1 \leq p \leq \infty
$$

ou seja, $W^{1, p}(I) \subset L^{\infty}(I)$ com injeção contínua para todo $1 \leq p \leq \infty$. Adicionalmente, quando I é limitado temos:

(i) $W^{1, p}(I) \subset C(\bar{I})$ é compacta para $1<p \leq \infty$

(ii) $W^{1,1}(I) \subset L^{q}(I)$ é compacta para $1 \leq p<\infty$. 
Corolário 1.9.1. (Derivação do Produto) Sejam $u, v \in W^{1, p} \cdot \operatorname{com} 1 \leq p \leq \infty$. Então $u v \in W^{1, p}(I) e$

$$
(u v)^{\prime}=u^{\prime} v+u v^{\prime}
$$

Portanto, temos também a regra de integração por partes:

$$
\int_{y}^{x} u^{\prime} v=u(x) v(x)-u(y) v(y)-\int_{y}^{x} u v^{\prime}, \forall x, y \in \bar{I} .
$$

Corolário 1.9.2. (Derivação de uma Composição) Seja $G \in C^{1}(\mathbb{R})$ tal que $G(0)=0 e$ seja $u \in W^{1, p}$. Então

$$
G \circ u \in W^{1, p}(I) \text { e }(G \circ u)^{\prime}=\left(G^{\prime} \circ u\right) u^{\prime} .
$$

Definição 1.9.2. Dados um inteiro $m \geq 2$ e um número real $1 \leq p \leq \infty$, definimos $o$ espaço $W^{m, p}$ por

$$
W^{m, p}(I)=\left\{u \in W^{m-1, p}(I), u^{\prime} \in W^{m-1, p}(I)\right\}
$$

Denota-se por $H^{m}(I)$ o espaço $W^{m, 2}(I)$.

Mostra-se que $u \in W^{m, p}(I)$ se e somente se existem $m$ funções $g_{1}, g_{2}, \ldots, g_{m} \in L^{p}(I)$ tais que

$$
\int_{I} u D^{j} \varphi=(-1)^{j} \int_{I} g_{j} \varphi \forall \varphi \in C_{c}^{\infty}(I), \forall j=1,2, \ldots, m .
$$

Quando $u \in W^{m, p}(I)$, consideraremos $u^{\prime}=g_{1},\left(u^{\prime}\right)^{\prime}=g_{2}, \ldots, D^{m} u=g_{m}$

O espaço $W^{m, p}$ está dotado da norma:

$$
\|u\|_{W^{m, p}}=\|u\|_{L^{p}}+\sum_{\alpha=1}^{m}\left\|D^{\alpha} u\right\|_{L^{p}}
$$

Em $H^{m}$ temos o produto escalar dado por:

$$
(u, v)_{H^{m}}=(u, v)_{L^{2}}+\sum_{\alpha=1}^{m}\left(D^{\alpha} u, D^{\alpha} v\right)_{L^{2}}
$$

A norma $\|u\|_{W^{m, p}}$ é equivalente a \|\|$u\|==\| u\left\|_{L^{p}}+\right\| D^{m} u \|_{L^{p}}$

Observação 1.9.1. Podemos estender algumas das propriedades obtidas para $W^{1, p}$, como por exemplo, $W^{m, p}(I) \subset C^{m-1}(\bar{I})$ com injeção contínua.

Definição 1.9.3. Dado $1 \leq p<\infty$ definimos o espaço $W_{0}^{1, p}$ como o fecho de $C_{c}^{1}(I)$ em $W^{1, p}(I)$. 
Denotaremos por $H_{0}^{1}(I)$ o conjunto $W_{0}^{1,2}$.

Pelo Teorema 1.9.5 sabemos que $C_{c}^{1}(\mathbb{R})$ é denso em $W^{1, p}(\mathbb{R})$ assim, $W_{0}^{1, p}(\mathbb{R})=$ $W^{1, p}(\mathbb{R})$.

O teorema seguinte caracteriza as funções de $W_{0}^{1, p}(I)$.

Teorema 1.9.7. Seja $u \in W^{1, p}(I)$, então $u \in W_{0}^{1, p}(I)$ se e somente se $u=0$ sobre $\partial I$.

Teorema 1.9.8. (Desigualdade de Poincaré) Se I é limitado, então existe uma constante $C$, dependendo de $|I|$, tal que

$$
\|u\|_{W^{1, p}} \leq C\left\|u^{\prime}\right\|_{L^{p}} \quad \forall u \in W_{0}^{1, p}(I)
$$

ou seja, a norma $\|u\|_{W^{1, p}}$ é equivalente $\left\|u^{\prime}\right\|_{L^{p}}$ no espaço $W_{0}^{1, p}(I)$.

\subsection{Existência e Unicidade de Solução Para Proble- mas de Contorno}

O objetivo desta seção é estudar a existência e unicidade de solução para problemas de contorno da forma:

$$
\left\{\begin{array}{l}
-u^{\prime \prime}+u=f \quad \text { em } I=(0,1) \\
u(0)=u(1)=0
\end{array}\right.
$$

onde $f \in C(\bar{I})$ ou $f \in L^{2}(I)$. As condiçōes de contorno $u(0)=u(1)=0$ são chamadas condições de Dirichlet (homogêneas).

Uma solução clássica (forte) de 1.37 é uma função $u$ de classe $C^{2}$ em [0,1] que verifica 1.37 no sentido usual. Uma função $u \in H_{0}^{1}(I)$ que verifica

$$
\int_{0}^{1} u^{\prime} v^{\prime}+\int_{0}^{1} u v=\int_{0}^{1} f v, \forall v \in H_{0}^{1}(I)
$$

é uma solução fraca de 1.37 .

Definição 1.10.1. Uma forma bilinear $a(u, v): H \times H \rightarrow \mathbb{R}$ é:

(i) contínua se existe uma constante $C$ tal que $|a(u, v)| \leq C|u \| v| \forall u, v \in H$ 
(ii) coerciva se existe uma constante $\alpha>0$ tal que $a(u, v) \geq \alpha|v|^{2} \quad \forall v \in H$

Lema 1.10.1. (Lax-Milgram) Seja a $(u, v)$ uma forma bilinear, contínua e coerciva. Então para todo $\varphi \in H^{\prime}$ existe $u \in H$ único tal que

$$
a(u, v)=\langle\varphi, v\rangle \forall v \in H
$$

E ainda, se a é simétrica, então u se caracteriza pela propriedade

$$
u \in H \quad e \frac{1}{2} a(u, u)-\langle\varphi, u\rangle=\min _{v \in H}\left\{\frac{1}{2} a(v, v)-\langle\varphi, v\rangle\right\}
$$

Teorema 1.10.1.

(i) Toda solução clássica de 1.37 é uma solução fraca de 1.37 .

(ii) Existe uma única solução fraca de 1.37.

(iii) A solução fraca obtida é de classe $C^{2}$, e portanto ela é uma solução clássica de 1.37.

Prova: A prova de (i) é simples. De fato, seja $u$ é uma solução forte de 1.37. Entāo multiplicando 1.37 por $v \in C^{1}[0,1]$ e integrando por partes, temos que $u$ verifica 1.38.

Prova de 2) Aplica-se o Lema 1.10.1 no espaço de Hilbert $H=H_{0}^{1}(I)$, com a forma bilinear

$$
a(u, v)=\int_{I} u^{\prime} v^{\prime}+\int_{I} u v=(u, v)_{H^{1}}
$$

e a forma linear $\varphi: v \rightarrow \int_{I} f v$.

Prova de 3) Observemos inicialmente que se $f \in L^{2}$ e se $u \in H_{0}^{1}$ é uma solução fraca, então $u \in H^{2}$. De fato, pois como $u$ é solução fraca, então

$$
\int u^{\prime} v^{\prime}=\int(f-u) v \quad \forall v \in C_{c}^{1}
$$

Com isso, temos que $u^{\prime} \in H^{1}$, já que $(f-u) \in L^{2}$ e, portanto $u \in H^{2}$. E ainda, se $f \in C(\bar{I})$ então a solução fraca $u \in C^{2}(\bar{I})$. De fato $\left(u^{\prime}\right)^{\prime} \in C(\bar{I})$ e então $u^{\prime} \in C^{1}(\bar{I})$, portanto $u \in C^{2}(\bar{I})$. E para finalizar, provaremos que uma solução fraca $u$ de 1.37 tal que $u \in C^{2}(\bar{I})$ é também uma solução clássica de 1.37. Para isso, integrando por partes 1.38, obtemos

$$
\int_{0}^{1}\left(-u^{\prime \prime}+u-f\right) v=0, \quad \forall v \in C^{1}[0,1], \quad v(0)=v(1)=0
$$


isto é,

$$
\int_{0}^{1}\left(-u^{\prime \prime}+u-f\right) v=0, \quad \forall v \in C_{c}^{1}(0,1)
$$

Como $C_{c}^{1}(0,1)$ é denso em $L^{2}(0,1)$, segue que $-u^{\prime \prime}+u=f$ quase sempre em $(0,1)$. $\square$

Lema 1.10.2. (Stampacchia) Seja a $(u, v)$ uma forma bilinear, contínua e coerciva. Seja $K$ um convexo, fechado e não vazio. Dado $\varphi \in H^{\prime}$ existe $u \in K$ único tal que

$$
a(u, v-u) \geq(\varphi, v-u\rangle \forall v \in H .
$$

E ainda, se a é simétrica, então u se caracteriza pela propriedade

$$
u \in K e \frac{1}{2} a(u, u)-\langle\varphi, u\rangle=\min _{v \in K}\left\{\frac{1}{2} a(v, v)-\langle\varphi, v\rangle\right\}
$$

Exemplo 1.10.1. (Condição de Dirichlet não homogênea) Resolver o seguinte problema

$$
\left\{\begin{array}{l}
-u^{\prime \prime}+u=f \quad \text { em } I=(0,1) \\
u(0)=\alpha, u(1)=\beta
\end{array}\right.
$$

onde $\alpha, \beta \in \mathbb{R}$ e f é uma função dada.

Prova: Tomemos em $H^{1}$ o convexo fechado

$$
K=\left\{v \in H^{1}(I) ; v(0)=\alpha, v(1)=\beta\right\}
$$

Se $u$ é uma solução clássica de 1.43, então ela é também uma solução fraca, e portanto:

$$
\int_{I} u^{\prime}(v-u)^{\prime}+\int_{I} u(v-u)=\int_{I} f(v-u) \quad \forall v \in K
$$

Em particular, temos que:

$$
\int_{I} u^{\prime}(v-u)^{\prime}+\int_{I} u(v-u) \geq \int_{I} f(v-u) \quad \forall v \in K
$$

Para obter a desigualdade acima, basta aplicar o Lema (1.10.2), tomando a forma bilinear

$$
a(u, v)=\int_{I} u^{\prime} v^{\prime}+\int_{I} u v=(u, v)_{H^{1}}
$$

e a forma linear $\varphi: v \rightarrow \int_{I} f v$. Logo, existe $u \in k$ única, tal que

$$
\int_{I} u^{\prime}(v-u)^{\prime}+\int_{I} u(v-u) \geq \int_{I} f(v-u) \quad \forall v \in K
$$

Tomemos nesta desigualdade $v=u \pm w$ onde $w \in H_{0}^{1}(I)$, para recuperar a solução clássica.u 
Exemplo 1.10.2. (Problema de Sturm-Liouville)

$$
\left\{\begin{array}{l}
-\left(p u^{\prime}\right)^{\prime}+q u=f \quad \text { em } I=(0,1) \\
u(0)=u(1)=0
\end{array}\right.
$$

onde $p \in C^{1}(\bar{I}), q \in C(\bar{I})$ ef $f L^{2}(I)$ com

$$
p(x) \geq \alpha>0 \quad \forall x \in \bar{I}
$$

Prova:

Se $u$ é uma solução clássica de 1.44, então ela é também uma solução fraca, e portanto:

$$
\int_{I} p u^{\prime} v^{\prime}+\int_{I} q u v=\int_{I} f v \quad \forall v \in H_{0}^{1}(I)
$$

Tomemos a forma bilinear

$$
a(u, v)=\int_{I} p u^{\prime} v^{\prime}+\int_{I} q u v
$$

e o espaço funcional $H_{0}^{1}$. Logo, pelo Lema (1.10.1) existe $u \in H_{0}^{1}$ única, tal que

$$
a(u, v)=\int_{I} f v \quad \forall v \in H_{0}^{1}(I) .
$$

Dessa forma, $p u^{\prime} \in H^{1}$. Se $u^{\prime}=\frac{1}{p} p u^{\prime}$, então $u \in H^{2}$. Portanto $u$ é uma solução clássica. $\square$ 


\section{Capítulo 2}

\section{Positividade e Critérios de}

\section{Comparação Abstratos}

O objetivo deste capítulo é tornar simples a verificação de condições suficientes para que soluçōes de problemas semilineares possam ser comparadas. No capítulo 4, aplicaremos os resultados obtidos aqui à equação do calor e aos sistemas de equações diferenciais ordinárias.

\subsection{Espaços de Banach Ordenados e Positividade}

Para que métodos de comparação possam ser desenvolvidos, vamos introduzir as propriedades básicas que uma ordem deve satisfazer em um Espaço de Banach.

Definição 2.1.1. Um espaço de Banach pré-ordenado é um par $(X, \leq)$, onde $X$ e um espaço de Banach e $\leq$ é uma relação em $X$ que satisfaz:

i) $x \leq y$ implica $x+z \leq y+z, x, y, z \in X$.

ii) $x \leq y$ e $y \leq z$ implicam $x \leq z$.

iii) $x \leq y$ implica $\lambda x \leq \lambda y$, para $x, y \in X$, e número real $\lambda \geq 0$.

iv) $O$ "cone positivo" $C=\{x \in X, x \geq 0\}$ é fechado em $X$. 
Se ainda,

$$
C \cap-C=\{0\}
$$

dizemos que $X$ é um espaço de Banach ordenado

Observação 2.1.1.

i) Observe que $x \leq y$ é equivalente a $y-x \geq 0$. Observe ainda que $x \leq 0$ se e somente se $0 \leq-x$ e que o cone $C$ é convexo. Note ainda que se $\lambda<\mu$ e $x \geq 0$ então $0 \leq(\mu-\lambda) x$ e $\lambda x \leq \mu x$.

ii) Todo subespaço fechado de um espaço de Banach ordenado é também um espaço de Banach ordenado com a ordem induzida.

iii) $S e\left(X, \leq_{X}\right) e\left(Y, \leq_{Y}\right)$ são espaços de Banach ordenados, então $X \times Y$ com a ordem definida por $(a, b) \leq_{X \times Y}(x, y)$ se e somente se $a \leq_{X} x$ eb $\leq_{Y} y$, é um espaço de Banach ordenado.

iv) Para $1 \leq p \leq \infty, X=L^{p}(\Omega)$, com a ordem " $f \leq g$ se e somente se $f(x) \leq g(x)$ quase sempre" é espaço de Banach pré-ordenado.

Definiremos agora o que entendemos por transformações que preservam ordem.

Definição 2.1.2. Sejam $(X, \leq)(Y, \preceq)$ espaços de Banach pré-ordenados. Uma função $T: D(T) \subset X \rightarrow Y$ é dita crescente se e somente se $x \leq y, x, y \in D(T)$, implica $T(x) \preceq T(y)$ e é chamada positiva se e somente se $x \leq 0, x \in D(T)$, implica $T(x) \preceq 0$.

Observação 2.1.2. Se na definição acima $T$ é linear então ambos conceitos coincidem.

Lema 2.1.1. Seja $(X, \leq)$ um espaço de Banach pré-ordenado e $f \in L^{1}\left(\left(t_{0}, t_{1}\right), X\right)$, tal que $f(t) \geq 0$ para quase todo $t \in\left(t_{0}, t_{1}\right)$. Então, $\int_{t_{0}}^{t_{1}} f(s) d s \geq 0$.

Prova: Como a integral é um operador linear contínuo entre $L^{1}\left(\left(t_{0}, t_{1}\right), X\right)$ e $X$ e 0 cone $C$ é fechado, é suficiente provar o resultado para $f$ em um subconjunto denso de $L^{1}\left(\left(t_{0}, t_{1}\right), X\right)$. Se $f \in C\left(\left[t_{0}, t_{1}\right], X\right)$, pelo teorema do valor médio para integral,

$$
\int_{t_{0}}^{t_{1}} f(s) d s=\left(t_{1}-t_{0}\right) \varlimsup \sum_{i=1}^{n} f\left(\zeta_{1}\right) \frac{\left(t_{i}-t_{i-1}\right)}{\left(t_{1}-t_{0}\right)} \in\left(t_{1}-t_{0}\right) \overline{c o}\left(f\left(\left[t_{0}, t_{1}\right]\right)\right),
$$

onde

$$
\overline{c o}\left(f\left(\left[t_{0}, t_{1}\right]\right)\right)
$$


é o fecho da envoltória convexa da imagem de $f$. Se $f(t) \geq 0$, para todo $t$, então sua imagem está em $C$ e então $\overline{c o}\left(f\left(\left[t_{0}, t_{1}\right]\right)\right) \subset C$, pois $C$ é convexo e $\overline{c o}\left(f\left(\left[t_{0}, t_{1}\right]\right)\right)$ é o menor convexo tal que $\left\{f(t): t \in\left[t_{0}, t_{1}\right]\right\} \subset \overline{c o}\left(f\left(\left[t_{0}, t_{1}\right]\right)\right) \cdot \square$

Definição 2.1.3. Seja $(X, \leq)$ um espaço de Banach pré-ordenado. Podemos definir uma ordem "”"(que chamaremos ordem dual) no dual $X$ ' de $X$ da seguinte forma: Dados $x^{\prime}, y^{\prime} \in X^{\prime}$ dizemos que $x^{\prime} \preceq y^{\prime}$ se e somente se

$$
\left\langle x^{\prime}, x\right\rangle_{X^{\prime}, X} \leq\left\langle y^{\prime}, x\right\rangle_{X^{\prime}, X}, \quad \forall 0 \leq x \in X
$$

Temos que $\left(X^{\prime}, \preceq\right)$ é um espaço de Banach pré-ordenado.

As propriedades i), ii), iii) de espaços de Banach pré-ordenados são facilmente verificadas. A propriedade iv) é verificada da seguinte forma: Seja $0 \preceq x_{n}^{\prime} \in X^{\prime}$ uma seqüência convergente para $x^{\prime} \in X^{\prime}$. Então, se $0 \leq x \in X, 0 \leq\left\langle x_{n}^{\prime}, x\right\rangle_{X^{\prime}, X} \rightarrow\left\langle x^{\prime}, x\right\rangle_{X^{\prime}, X}$ e $0 \leq\left\langle x^{\prime}, x\right\rangle_{X^{\prime}, X}$. Segue que $0 \preceq x^{\prime}$.

Definição 2.1.4. Seja $\left(X_{1} \leq\right)$ um espaço de Banach pré-ordenado e seja “ $\preceq$ " a ordem dual em $X^{\prime}$. Dizemos que " $\leq " e$ "\"são compatíveis se

$$
\left\langle x^{\prime}, x\right\rangle_{X^{\prime}, X} \geq 0, \quad \forall 0 \preceq x^{\prime} \in X^{\prime} \quad \Rightarrow \quad x \geq 0
$$

Consideremos o espaço $L^{q}(\Omega), q \geq 1$ com a ordem natural e $q^{\prime}$ tal que $1 / q+1 / q^{\prime}=1$. A ordem dual em $\left(L^{q}(\Omega)\right)^{\prime}=L^{q^{\prime}}(\Omega)$, coincide com a ordem natural de $L^{q^{\prime}}(\Omega)$. De fato, pois se $x^{\prime}, y^{\prime} \in\left(L^{q}(\Omega)\right)^{\prime}$, temos:

$$
\begin{aligned}
x^{\prime} \preceq y^{\prime} & \Leftrightarrow\left\langle x^{\prime}, x\right\rangle_{\left(L^{q}(\Omega)\right)^{\prime},\left(L^{q}(\Omega)\right)} \leq\left\langle y^{\prime}, x\right\rangle_{\left(L^{q}(\Omega)\right)^{\prime},\left(L^{q}(\Omega)\right)}, \quad \forall 0 \leq x \in\left(L^{q}(\Omega)\right) \\
& \Leftrightarrow 0 \leq\left\langle y^{\prime}, x\right\rangle_{\left(L^{q}(\Omega)\right)^{\prime},\left(L^{q}(\Omega)\right)}-\left\langle x^{\prime}, x\right\rangle_{\left(L^{q}(\Omega)\right)^{\prime},\left(L^{q}(\Omega)\right)} \quad \forall 0 \leq x \in\left(L^{q}(\Omega)\right) \\
& \Leftrightarrow \int\left(y^{\prime}-x\right)^{\prime} x \geq 0, \quad \forall 0 \leq x \in L^{q}(\Omega) \\
& \Leftrightarrow y^{\prime}-x^{\prime} \geq 0 \quad \text { em quase toda parte } \\
& \Leftrightarrow y^{\prime} \preceq x^{\prime} \quad \text { em quase toda parte. }
\end{aligned}
$$


Temos também que $\approx$ ordem de $L^{q}(\Omega)$ é compatível com a ordem dual de $\left(L^{q}(\Omega)\right)^{\prime}$. Isto é um conseqüência do fato que se $v \in L^{q^{\prime}}(\Omega)$, então

$$
\int_{\Omega} u(x) v(x) d x \geq 0 \quad \forall u \in L^{q}(\Omega), 0 \leq u(x), \text { q.s. em } \Omega \Longleftrightarrow v(x) \geq 0 \text { q.s. em } \Omega \text {. }
$$

Lema 2.1.2. Suponha que $X$ é um espaço de Banach reflexivo e $X^{\prime}$ o seu dual. Seja $\leq$ uma ordem em $X$, “ ordem definida em $X^{\prime \prime}$ por “ $X^{\prime \prime}$ são identificados.

Prova: Temos que $x^{\prime \prime}$ e menor ou igual a $y^{\prime \prime}$ relativamente a ordem induzida em $X^{\prime \prime}$ pela ordem de $X^{\prime}$ se e somente se

$$
\left(x^{\prime \prime}, x^{\prime}\right\rangle_{X^{\prime \prime}, X^{\prime}} \preceq\left(y^{\prime \prime}, x^{\prime}\right\rangle_{X^{\prime \prime}, X^{\prime}}, \quad \forall 0 \preceq x^{\prime} \in X^{\prime}
$$

E quando identificamos $X$ e $X^{\prime \prime}$ temos

$$
\left\langle x^{\prime}, x\right\rangle_{X^{\prime}, X}=\left\langle x^{\prime \prime}, x^{\prime}\right\rangle_{X^{\prime \prime}, X^{\prime}} \leq\left\langle y^{\prime \prime}, x^{\prime}\right\rangle_{X^{\prime \prime}, X^{\prime}}=\left\langle x^{\prime}, y\right\rangle_{X^{\prime}, X}, \quad \forall 0 \preceq x^{\prime} \in X^{\prime}
$$

o que implica:

$$
0 \leq\left\langle x^{\prime}, y-x\right\rangle_{X^{\prime}, X}, \quad \forall 0 \preceq x^{\prime} \in X^{\prime}
$$

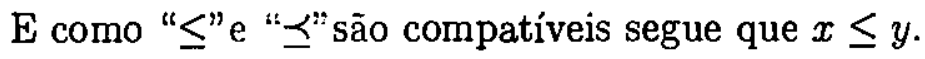

Lema 2.1.3. Sejam $\left(E, \leq_{E}\right)$ e $\left(F, \leq_{F}\right)$ espaços de Banach pré-ordenados e $A: D(A) \subset$ $E \rightarrow F$ uma transformação linear positiva tal que $\overline{D(A)}=E$. Se C denota o cone positivo de $E$, assuma que $D(A) \cap C$ é denso em $C$. Então $A^{\prime}: D\left(A^{\prime}\right) \subset F^{\prime} \rightarrow E^{\prime}$ é uma transformação linear positiva.

Prova: Note que $A$ é densamente definida e portanto $A^{\prime}$ está bem definida. Como $A$ é positiva temos que $0 \leq_{F} A u$ sempre que $0 \leq_{E} u$ e $u \in D(A)$. Assim se $v \in D\left(A^{\prime}\right)$, $0 \leq F_{F^{\prime}} v^{\prime}$ então da definição de $A^{\prime}$ e de ordem dual em $F^{\prime}$ obtemos que

$$
0 \leq\left\langle A u, v^{\prime}\right\rangle_{F, F^{\prime}}=\left\langle u, A^{\prime} v^{\prime}\right\rangle_{E, E^{\prime}}
$$

Portanto, temos que para $0 \leq_{E} u$ e $u \in D(A), 0 \leq\left\langle u, A^{\prime} v^{\prime}\right\rangle_{E, E^{\prime}}$. Segue da densidade de $C \cap D(A)$ em $C$ que $0 \leq_{E^{\prime}} A^{\prime} v_{\square}$ 


\subsection{A Equação Linear}

Os resultados a seguir estabelecem a equivalência entre a positividade do resolvente do gerador de um semigrupo e a positividade do semigrupo.

Proposição 2.2.1. Suponha que $(X, \leq)$ é um espaço de Banach pré-ordenado, $\{T(t)$ : $t \geq 0\}$ um $C_{0}$-semigrupo em $X$ e $A$ o seu gerador. Seja $M>0$ e $\omega \in \mathbb{R}$ tais que $\|T(t)\|_{L(X)} \leq M \mathrm{e}^{\omega t}$. Assuma que existe $\lambda_{0}>0$ tal que $(\lambda-A)^{-1}$ é crescente para todo $\lambda>\lambda_{0}$. Então, $T(t)$ é crescente para todo $t \geq 0$.

Reciprocamente, se $X \ni u_{0} \mapsto T(t) u_{0} \in C([0, \infty), X)$ é crescente para todo $t \geq 0$, então $(\lambda-A)^{-1}$ é crescente para todo $\lambda>\omega$.

Prova: Pelo Teorema 1.2.6 temos que:

$$
T(t) u_{0}=\lim _{n \rightarrow \infty}\left[\frac{n}{t}\left(\frac{n}{t}-A\right)^{-1}\right]^{n} u_{0} \quad \forall u_{0} \in X \text { e } t \geq 0
$$

Tomando $u_{0} \geq 0$ queremos provar que $T(t) u_{0} \geq 0, t \geq 0$. O caso $t=0$ é trivial, assumimos que $t>0$. Por hipótese, para $\frac{n}{t}>\lambda_{0}$, temos que $\left(\frac{n}{t}-A\right)^{-1} u_{0} \geq 0$. E assim, obtemos que

$$
\left[\frac{n}{t}\left(\frac{n}{t}-A\right)^{-1}\right]^{n} u_{0} \geq 0, \forall n>t \lambda_{0} .
$$

Portanto, obtemos uma seqüência de elementos em $C$ que converge para $T(t) u_{0}$ Segue do fato que o cone positivo é fechado que $T(t) u_{0} \geq 0$.

A recíproca segue do fato que a integral é um operador positivo e de

$$
(\lambda-A)^{-1}=\int_{0}^{\infty} T(t) \mathbf{e}^{-\lambda t} d t \geq 0
$$

para todo $\lambda>\omega_{\cdot \square}$

Observaçāo 2.2.1.

i) Observe que a hipótese acima sobre $A$ é equivalente a um resultado de positividade para o problema "elíptico" $\lambda u-A u=f$ : para todo $\lambda>\lambda_{0}$ e para todo $f \geq 0$, a solução satisfaz $u \geq 0$.

Equivalentemente, temos o resultado de comparação: para todo $\lambda>\lambda_{0}$ ef $\geq g$, se $\lambda u-A u=f$ e $\lambda v-A v=g$, então $u \geq v$. 
ii) Da mesma forma observe que a conclusão da proposição é equivalente a: para $u_{0} \geq 0$ a solução da equação linear homogênea

$$
\left\{\begin{array}{l}
u_{t}=A u \\
u(0)=u_{0} \in X
\end{array} \quad \text { verifica } \quad u(t) \geq 0 \text { para todo } t \geq 0 .\right.
$$

ou equivalentemente, se $u_{0} \geq v_{0}$, então $u(t) \geq v(t)$ para todo $t \geq 0$, onde $u$ e $v$ são soluções da equação linear homogênea com dados iniciais $u_{0}$ e $v_{0}$ respectivamente.

iii) Se $A$ verifica as hipóteses acima, então o mesmo acontece com $A+\mu$, para todo $\mu \in \mathbb{R}$.

Corolário 2.2.1. Suponha que $(X, \leq)$ é um espaço de Banach pré-ordenado, e seja $A$ o gerador de um $C_{0}$-semigrupo $\{T(t): t \geq 0\}$ tal que $\|T(t)\|_{L(X)} \leq M \mathrm{e}^{\omega t}$. Assuma que para todo $\lambda>\lambda_{0},(\lambda-A)^{-1}$ é crescente.

i) Seja $u_{0} \in D(A)$ tal que $A u_{0} \leq 0$. Então se $u(t)=T(t) u_{0}$, temos

$$
A u(t) \leq 0, \quad u_{t}(t) \leq 0, \quad u(t) \leq u(s) \leq u_{0}
$$

para todo $0 \leq s \leq t$. O mesmo vale, com as desigualdades invertidas, se $A u_{0} \geq 0$.

ii) Seja $u_{0} \in X$ tal que $u_{0} \geq 0$ e sejam $\lambda$, $\mu$ números reais tais que $\lambda>\mu$. Então, se $u_{\lambda}(t)$ e $u_{\mu}(t)$ denotam respectivamente as soluções de $u_{t}=A u-\lambda u$ e $u_{t}=A u-\mu u$, com dado inicial $u_{0}$, então $0 \leq u_{\lambda}(t) \leq u_{\mu}(t)$ para todo $t$.

Prova: i) Seja $u_{0} \in D(A)$ tal que $A u_{0} \leq 0$. De

$$
\frac{d}{d t} T(t) u_{0}=T(t) A u_{0}
$$

e da Proposição 2.2.1 segue que

$$
\begin{gathered}
A u(t)=A T(t) u_{0}=T(t) A u_{0} \leq 0 \\
u_{t}(t)=T(t) A u_{0} \leq 0
\end{gathered}
$$

E para finalizar tal item, temos que para todo $0 \leq s \leq t$,

$$
\int_{s}^{t} u_{t}(t) \leq 0 \Rightarrow u(t) \leq u(s)
$$

Analogamente, temos que: $u(s) \leq u_{0}$.

ii) Sabemos que $u_{\lambda}(t)=\mathrm{e}^{-\lambda t} T(t) u_{0}$ e $u_{\mu}(t)=\mathrm{e}^{-\mu t} T(t) u_{0}$. Como $\lambda<\mu$ implica $\lambda t \leq \mu t$, o resultado segue. $\square$ 
Corolário 2.2.2. Seja $(X, \leq)$ um espaço de Banach pré-ordenado e $A$ o gerador de um $C_{0}$-semigrupo $\{T(t): t \geq 0\}$. Assuma que existe $\lambda_{0} \in \mathbb{R}$ tal que para $\lambda>\lambda_{0},(\lambda-A)^{-1}$ é crescente.

Seja $u=u\left(t, u_{0}, f\right)$ a solução de

$$
\left\{\begin{array}{l}
u_{t}=A u+f(t) \\
u\left(t_{0}\right)=u_{0} \in X
\end{array}\right.
$$

$\operatorname{com} f \in L^{1}\left(\left(t_{0}, t_{1}\right), X\right)$.

Assuma $u_{0} \geq u_{1}$ e $f_{0}(t) \geq f_{1}(t)$ para quase todo $t \in\left(t_{0}, t_{1}\right)$, então $u\left(t, u_{0}, f_{0}\right) \geq$ $u\left(t, u_{1}, f_{1}\right)$ para todo $t \in\left(t_{0}, t_{1}\right)$. Em particular, se $u_{0} \geq 0$ e $f(t) \geq 0$ para quase todo $t \in\left(t_{0}, t_{1}\right)$, então $u\left(t, u_{0}, f\right) \geq 0$ para todo $t \in\left(t_{0}, t_{1}\right)$.

Prova: Observe que a solução é dada pela fórmula da variação das constantes

$$
u_{i}(t)=T\left(t-t_{0}\right) u_{i}+\int_{t_{0}}^{t} T(t-s) f_{i}(s) d s
$$

para $i=0,1$, e já sabemos que $T\left(t-t_{0}\right)$ é crescente. Por outro lado, para todo $t_{0}<s<$ $t<t_{1}, T(t-s) f_{0}(s) \geq T(t-s) f_{1}(s)$ e como a integral é crescente obtemos o resultado. $\square$

O resultado a seguir, garante que quando tratamos de semigrupos de operadores lineares positivos o cone positivo contém um subconjunto denso de funções suaves.

Lema 2.2.1. Seja $(X, \leq)$ um espaço de Banach pré-ordenado e A o gerador de um $C_{0}$-semigrupo $\{T(t): t \geq 0\}$ tal que $T(t)$ é positivo para todo $t \geq 0$. Então $C_{\infty}:=$ $\cap_{k \geq 1} D\left(A^{k}\right) \cap C$ é denso em $C$.

Prova: Como $T(t)$ é positivo para todo $t \geq 0$ segue que se $x \in C$, então $T(t) x \in C$.

Seja $\phi: \mathbb{R} \rightarrow \mathbb{R}^{+}$uma funçāo infinitamente diferenciável, com suporte contido compactamente em $(0, \infty)$. Dado $x \in C$ e $f=\int_{0}^{\infty} \phi(t) T(t) x d t$. Temos que $f \in D(A)$. De fato, dado $h>0$, fazendo uma mudança de variáveis, obtemos:

$$
\begin{aligned}
\lim _{h \rightarrow 0^{+}} h^{-1}[T(h) f-f] & =\lim _{h \rightarrow 0^{+}} h^{-1}\left\{\int_{h}^{\infty}[\phi(t-h)-\phi(t)] T(t) x d t-\int_{0}^{\infty} \phi(t) T(t) x d t\right\} \\
& =-\int_{0}^{\infty} \phi^{\prime}(t) T(t) x d t
\end{aligned}
$$

Portanto, $f \in D(A)$. E como - $\phi^{\prime}$ se comporta como a $\phi, A f$ tem as mesmas propriedades de $f$, logo $f \in D\left(A^{2}\right)$. Indutivamente, obtemos que $f \in D\left(A^{n}\right), \quad \forall n \geq 1$. 
Escolha $\phi$ tal que $\int_{0}^{\infty} \phi(t) d t=1$. e seja $f_{n}=\int_{0}^{\infty} n \phi(n t) T(t) x d s \geq 0$. Então

$$
\cap_{k \geq 1} D\left(A^{k}\right) \ni f_{n}=\int_{0}^{\infty} \phi(t) T(t / n) x d s \geq 0
$$

e $f_{n} \rightarrow x$ quando $n \rightarrow \infty$. Claramente $f_{n} \in C_{\infty}$ e o resultado segue. $\square$

Agora mostraremos que estes conceitos e resultados podem ser transferidos para as potências fracionárias de operadores setoriais.

Seja $-A$ um operador setorial e $X^{\alpha}$ os espaços de potência fracionárias associados a $-A$. Se $(X, \leq)$ é um espaço de Banach ordenado podemos definir em $X^{\alpha}, \alpha>0$, a ordem induzida por $X$; isto é, denotando por $C$ o cone positivo de $X$ definimos o cone positivo de $X^{\alpha}$ por $C_{\alpha}=C \cap X^{\alpha}$. Denotaremos por $\leq_{\alpha}$ a ordem induzida em $X^{\alpha}$ pela ordem de $X$.

É fácil verificar que $\left(X^{\alpha}, \leq_{\alpha}\right)$ é um espaço de Banach ordenado, de fato: as propriedades i) e ii) da Definição 2.1.1 são imediatas; a propriedade iii) segue do fato que se $x_{n} \rightarrow x$ em $X^{\alpha}$ então $x_{n} \rightarrow x$ em $X$ e do fato que $C$ é fechado.

$O$ resultado a seguir, resume as propriedades importantes das ordens em espaços de potência fracionária.

Proposição 2.2.2. Seja $(X, \leq)$ um espaço de Banach pré-ordenado, $-A: D(A) \subset X \rightarrow$ $X$ um operador setorial e $\{T(t): t \geq 0\} \subset L(X)$ o semigrupo gerado por A. Assuma que $T(t)$ é um operador positivo para cada $t \geq 0$. Então $\left(X^{\alpha}, \leq_{\alpha}\right)$ é um espaço de Banach ordenado, para todo $\alpha \geq 0$ e se $\alpha>\beta,{\overline{C_{\alpha}}}^{X^{\beta}}=C_{\beta}$; isto é, se $\phi \geq 0$ em $X^{\beta}$, então existe $\phi_{n} \geq 0$ em $X^{\alpha}$ tal que $\phi_{\mathfrak{n}} \rightarrow \phi e m X^{\beta}$.

Adicionalmente, se $\|T(t)\|_{L(X)} \leq M \mathrm{e}^{\omega t}$ e $(\lambda-A)^{-1}: X^{\alpha} \rightarrow X^{\alpha}$ é crescente para todo $\lambda>\omega, \alpha \geq 0$, e conseqüentemente a transformação

$$
X^{\alpha} \ni u_{0} \mapsto T(t) u_{0} \in C\left([0, \infty), X^{\alpha}\right)
$$

é linear crescente.

Prova: A única afirmativa que precisa ser verificada é

$$
{\overline{C_{\alpha}}}^{X^{\beta}}=C_{\beta}, \alpha>\beta \geq 0 .
$$


Assuma que $f \in C_{\beta}$ seja $g(t)=\mathrm{e}^{-A t} f$. Então, pelo efeito de regularização, $g(t) \in X^{\alpha}$. De $f \in C_{\beta}$ e $T(t)=\mathrm{e}^{-A t}$ é crescente, segue que $g(t) \in C_{\beta}$. Portanto, $g(t) \in C_{\beta} \cap X^{\alpha}=C_{\alpha}$ e $g(t) \rightarrow f$ em $X^{\beta}$, quando $t \rightarrow 0$. Conseqüentemente, ${\overline{C_{\alpha}}}^{X^{\beta}}=C_{\beta \cdot \square}$

Observe que a propriedade acima dos cones positivos mostra a consistência das definiçōes " $f \geq 0$ ", independentemente do espaço $X^{\alpha}$ no qual $f$ está. Portanto, de agora em diante, não faremos distinção entre as ordens $\leq_{\alpha}, \alpha \geq 0$.

Como nos Corolários 2.2.1 e 2.2.2, obtemos resultados de comparação e positividade para a equação linear não homogênea

$$
\left\{\begin{array}{l}
u_{t}=A u+f(t) \\
u(0)=u_{0} \in X^{\alpha} .
\end{array}\right.
$$

Corolário 2.2.3. Seja $\left(X_{i} \leq\right)$ um espaço de Banach pré-ordenado e $\{T(t): t \geq 0\} \subset$ $L(X)$ um semigrupo de operadores lineares positivos com gerador $A$.

i) Seja $u_{0} \in D(A)$ tal que $A u_{0} \leq 0$. Então, se $u(t)=T(t) u_{0}$ temos

$$
A u(t) \leq 0, \quad u_{t}(t) \leq 0, \quad u(t) \leq u(s) \leq u_{0}
$$

para todo $0 \leq s \leq t$. O mesmo é verdade, com as desigualdades invertidas, se $A u_{0} \geq 0$.

ii) Seja $u_{0} \in X$ tal que $u_{0} \geq 0$ e seja $\lambda, \mu \in \mathbb{R}$ tais que $\lambda>\mu$. Então, se $u_{\lambda}(t)$ e $u_{\mu}(t)$ denotam respectivamente as soluções de $u_{t}=A u-\lambda u=0$ e $u_{t}=A u-\mu u=0$, com dado inicial $u_{0}$, então $0 \leq u_{\lambda}(t) \leq u_{\mu}(t)$ para todo $t$.

iii) Se $-A$ é setorial, para qualquer $\alpha \geq 0$, a transformação

$$
X^{\alpha} \times L^{1}\left(\left(t_{0}, t_{1}\right), X^{\alpha}\right) \ni\left(u_{0}, f\right) \mapsto u \in C\left(\left[t_{0}, t_{1}\right], X^{\alpha}\right)
$$

é crescente.

\subsection{Alguns Operadores com Resolvente Positivo}

Os resultados das seções precedentes são dependentes da possibilidade de encontrarmos geradores de semigrupos fortemente contínuos, $A$, cujo operador resolvente, $(\lambda-A)^{-1}$, seja um operador positivo para todo $\lambda \in(\omega, \infty)$. Nesta seção asseguramos que há uma classe interessante de operadores com esta propriedade. 
Lema 2.3.1. Seja $H$ um espaço de Hilbert e $f \in H$. Suponha que existe $\tilde{f} \in H$ tal que $\|\tilde{f}\| \leq\|f\|$ e que

$$
\langle\tilde{f}, f\rangle \geq|\langle f, f\rangle|
$$

Então $f=\tilde{f}$.

Prova: Sabemos que $\|\tilde{f}\| \leq\|f\|$ e que

$$
\|f\|^{2}=|\langle f, f\rangle| \leq\langle\tilde{f}, f\rangle=|\langle f, f\rangle| \leq\|\tilde{f}\|\|f\| .
$$

Segue que $\|f\|=\|\tilde{f}\|$ e

$$
0 \leq\langle f-\tilde{f}, f-\tilde{f}\rangle=2\|f\|^{2}-2\langle\tilde{f}, f\rangle \leq 0
$$

implica que $f=\tilde{f} \cdot \square$

Definição 2.3.1. Seja $H$ um espaço de Hilbert ordenado e $C$ o seu cone positivo. Dizemos que um operador auto-adjunto $A: D(A) \subset H \rightarrow H$ é positivo, se $\langle A u, u\rangle \geq 0$ para todo $u \in D(A)$. E ainda, dizemos que $\langle A+\alpha)^{-1}$ é crescente se $(A+\alpha)^{-1} f \in C$ sempre que $f \in C$.

Teorema 2.3.1. Sejam $H, A$ e $C$ como acima. Assuma que $H$ possui um subconjunto denso $D$ tal que:

- $(A+\alpha)^{-1} D \subset D$

- Para cada $d \in D$ podemos definir $|d| \in D \cap C$ e esta relação satisfaz: Um elemento $d \in D$ está em $C$ se e somente se $d=|d|$ e $\|d\|=\||d|\| ;$

- $\langle|d|, g\rangle \geq|\langle d, g\rangle|, \quad \forall d \in D, \forall g \in C$.

Considere as seguintes afirmativas:

(i) Se $u \in D\left(A^{\frac{1}{2}}\right)$ então $|u| \in D\left(A^{\frac{1}{2}}\right) e$

$$
\left\langle A^{\frac{1}{2}}|u|, A^{\frac{1}{2}}|u|\right\rangle \leq\left\langle A^{\frac{1}{2}} u, A^{\frac{1}{2}} u\right\rangle .
$$

(ii) $(A+\alpha)^{-1}$ é crescente para todo $\alpha>0$. 
Então (i) implica (ii).

Prova: $\operatorname{Em} D\left(A^{\frac{1}{2}}\right)$ adotamos o produto interno

$$
\langle f, g\rangle_{1}=\left\langle A^{\frac{1}{2}} f, A^{\frac{1}{2}} g\right\rangle+\alpha\langle f, g\rangle
$$

onde $\alpha>0$. Denotamos por $H^{\frac{1}{2}}$ o espaço de Hilbert $\left(D\left(A^{\frac{1}{2}}\right),\langle\cdot, \cdot\rangle_{I}\right)$.

Se $D \ni g \geq 0$ e $c=(A+\alpha)^{-1} g,\left(c \in D\right.$, pois $\left.(A+\alpha)^{-1} D \subset D\right)$

$$
\begin{aligned}
\langle|c|, c\rangle_{1} & =\left\langle|c|,(A+\alpha)^{-1} g\right\rangle_{1}=\left\langle A^{\frac{1}{2}}|c|, A^{\frac{1}{2}}(A+\alpha)^{-1} g\right\rangle+\alpha\left\langle|c|,(A+\alpha)^{-1} g\right\rangle \\
& =\left\langle(A+\alpha)|c|,(A+\alpha)^{-1} g\right\rangle=\langle|c|, g\rangle \geq|\langle c, g\rangle| \\
& =\left|\left\langle(A+\alpha) c,(A+\alpha)^{-1} g\right\rangle\right|=\left|\left\langle c,(A+\alpha)^{-1} g\right\rangle_{1}\right|=\left|\langle c, c\rangle_{1}\right| .
\end{aligned}
$$

Adicionalmente, pela propriedade (i)

$$
\begin{aligned}
\||c|\|_{1}^{2} & =\left\langle A^{\frac{1}{2}}|c|, A^{\frac{1}{2}}|c|\right\rangle+\alpha\||c|\|^{2} \\
& \leq\left\langle A^{\frac{1}{2}} c, A^{\frac{1}{2}} c\right\rangle+\alpha\|c\|^{2} \\
& =\|c\|_{1}^{2} .
\end{aligned}
$$

Usando o Lema 2.3.1 com $f=c$ e $\tilde{f}=|c|$ concluímos que se $g \in D \cap C$ então

$$
\left|\langle A+\alpha)^{-1} g\right|=\langle A+\alpha)^{-1} g
$$

e $(A+\alpha)^{-1} g \in C$. Da densidade de $D \cap C$ em $C$ e da continuidade de $(A+\alpha)^{-1}$, segue que

$$
\langle A+\alpha)^{-1} g \in C \forall g \in C .
$$

Portanto $(A+\alpha)^{-1}$ é crescente.

\subsection{A Equação Não Linear}

Nesta seção tratamos da comparação e positividade para problemas semilineares da forma

$$
\left\{\begin{array}{l}
u_{t}=A u+f(t, u) \\
u\left(t_{0}\right)=u_{0}
\end{array}\right.
$$


onde $A$ é o gerador de um semigrupo de operadores lineares e positivos. Esta seção está dividida em duas subseções: uma que trata o caso em que $A$ é o gerador de um $C_{0^{-}}$ semigrupo de operadores positivos e outra que trata do caso em que $A$ gera um semigrupo analítico de operadores positivos.

\subsubsection{O caso hiperbólico}

Nesta subseção tratamos da comparação e positividade para problemas semilineares da forma (2.2) no caso em que $A$ é gerador de um semigrupo fortemente contínuo de operadores lineares positivos em $X, f:\left[t_{0}, t_{1}\right) \times X \rightarrow X$ é contínua em $t$, localmente Lipschitz contínua em $u$. A solução de $(2.2)$ e denotada por $u_{f}\left(t, u_{0}\right)$.

Se $(X, \leq)$ é um espaço de Banach ordenado, o resultado a seguir estabelece condições sobre $f$ que asseguram a invariância do cone positivo $C$ de $X$.

Teorema 2.4.1. Seja $(X, \leq)$ um espaço de Banach ordenado e $\{T(t): t \geq 0\} \subset L(X)$ um $C_{0}$-semigrupo de operadores lineares positivos. Assuma que $f$ é tal que $f(t, x) \geq 0$ para todo $t \in\left[t_{0}, t_{1}\right)$ e $x \in X$. Então, $u_{0} \geq 0$ implica $u_{f}\left(t, u_{0}\right) \geq 0$, enquanto a solução existir.

Prova: Seja $\mathcal{F}$ o operador dado pela fórmula da variação das constantes; isto é,

$$
\mathcal{F}(u)=T\left(t-t_{0}\right) u_{0}+\int_{t_{0}}^{t} T(t-s) f(s, u(s)) d s .
$$

Analogamente a demonstração feita no Teorema 1.7.2, temos que $\mathcal{F}$ é uma contração em

$$
V=\left\{u \in C\left(\left[t_{0}, t_{0}+h\right], X\right),\left\|u(t)-u_{0}\right\|_{X} \leq \delta\right\},
$$

se $h$ e $\delta$ são suficientemente pequenos.

Tomando inicialmente $y_{0}(t)=u_{0} \geq 0$, então $f\left(s, y_{0}(s)\right) \geq 0$ e pelo Corolário 2.2.3 obtemos $y_{1}(t)=\mathcal{F}\left(y_{0}(t)\right) \geq 0$. Iterando, obtemos $y_{n}(t)=\mathcal{F}\left(y_{n-1}(t)\right) \geq 0$. Como $y_{n}(t)$ converge para $u(t)$ em $C\left(\left[t_{0}, t_{0}+h\right], X\right)$, então $u(t) \geq 0$ em $\left[t_{0}, t_{0}+h\right]$.

Assuma que $u$ é definido em $\left[t_{0}, t_{1}\right)$ e defina $t_{+}=\sup \left\{t \in\left[t_{0}, t_{1}\right), u(t) \geq 0\right\}$. Se $t_{+}<t_{1}$, então $t_{+}$é finita e pela continuidade, $u\left(t_{+}\right) \geq 0$, mas então o argumento acima contradiz a definição de $t_{+}$e então $t_{+}=t_{1 \cdot \square}$ 
Teorema 2.4.2. Com as notações acima, assuma que para todo $r>0$ existe uma constante $\beta=\beta(r)>0$ tal que para todo $t \in\left[t_{0}, t_{1}\right), f(t, \cdot)+\beta I$ é positiva sobre uma bola em $X$ de raio $r$.

Então, se $u_{0} \geq 0, u_{f}\left(t, u_{0}\right)$ é positiva enquanto existir.

Prova: Inicialmente escrevemos a equação (2.2) na forma

$$
u_{t}=(A-\beta I) u+f(t, u)+\beta u \text {. }
$$

Seja $\mathcal{F}$ o operador dado por

$$
\mathcal{F}(u)=T\left(t-t_{0}\right) \mathbf{e}^{-\beta\left(t-t_{0}\right)} u_{0}+\int_{t_{0}}^{t} T(t-s)(f(s, u(s))+\beta u(s)) d s .
$$

Temos que $\mathcal{F}$ é uma contração em $V=\left\{u \in C\left(\left[t_{0}, t_{0}+h\right], X\right),\left\|u(t)-u_{0}\right\|_{X} \leq \delta\right\}$ se $h$ e $\delta$ são suficientemente pequenos.

Dado $r=\left\|u_{0}\right\|_{X}+\delta$, existe $\beta=\beta\left(\left\|u_{0}\right\|_{X}+\delta\right)$ tal que $f(t, \cdot)+\beta I \geq 0$ na bola de raio $\left\|u_{0}\right\|_{X}+\delta$. Temos ainda que $T(t) \mathrm{e}^{-\beta t} \geq 0$.

Tomando inicialmente $y_{0}(t)=u_{0} \geq 0$ novamente temos $f\left(s, y_{0}(s)\right)+\beta y_{0}(s) \geq 0$ e então pelo Corolário 2.2 .3 obtemos $y_{1}(t)=\mathcal{F}\left(y_{0}(t)\right) \geq 0$. Iterando, $y_{n}(t)=\mathcal{F}\left(y_{n-1}(t)\right) \geq 0$ e como $y_{n}(t)$ converge para $u(t)$ em $C\left(\left[t_{0}, t_{0}+h\right], X\right)$, então $u(t) \geq 0$ em $\left[t_{0}, t_{0}+h\right]$.

O mesmo argumento usado anteriormente prova que a solução é positiva enquanto existir. $\square$

Relativamente a comparação, temos

Teorema 2.4.3. Suponha que $(X, \leq)$ é um espaço de Banach ordenado, e seja $\{T(t)$ : $t \geq 0\} \subset L(X)$ um $C_{0}$-semigrupo de operadores lineares positivos.

i) Assuma que para todo $t \in\left[t_{0}, t_{1}\right), f(t, \cdot)$ é crescente. Então $u_{0} \geq u_{1}$ implica $u_{f}\left(t, u_{0}\right) \geq$ $u_{f}\left(t, u_{1}\right)$ enquanto ambas existirem.

ii) Assuma que $f, g$ satisfazem $f(t, x) \geq g(t, x)$ para todo $t \in\left[t_{0}, t_{1}\right)$ e $x \in X$. Então, $u_{f}\left(t, u_{0}\right) \geq u_{g}\left(t, u_{0}\right)$ enquanto ambas existirem.

iii) Assuma que $f, g$ são tais que $x \geq y$ implica $f(t, x) \geq g(t, y)$. Então, $u_{0} \geq u_{1}$ implica $u_{f}\left(t, u_{0}\right) \geq u_{g}\left(t, u_{1}\right)$ enquanto ambas existirem.

Prova: i) Sabemos que $u_{i}(t)=u_{f}\left(t, u_{i}\right)$ é um ponto fixo de

$$
\mathcal{F}(u)=T\left(t-t_{0}\right) u_{i}+\int_{t_{0}}^{t} T(t-s) f(s, u(s)) d s
$$


em $V_{i}=\left\{u \in C\left(\left[t_{0}, t_{0}+h\right], X\right),\left\|u(t)-u_{i}\right\|_{X} \leq \delta\right\}$, para $h$ e $\delta$ suficientemente pequenos. Tomando como função inicial para as iterações $y_{i}^{0}(t)=u_{i}$, obtemos $y_{i}^{n}(t)=\mathcal{F}\left(y_{i}^{n-1}(t)\right) \mathrm{e}$ esta seqüência converge para $u_{i}(t)$ em $C\left(\left[t_{0}, t_{0}+h\right], X\right)$.

Mas $y_{0}^{0}(t) \geq y_{1}^{0}(t)$ e portanto $f\left(s, y_{0}^{0}(s)\right) \geq f\left(s, y_{1}^{0}(s)\right)$ e pelo Corolário 2.2.3, $y_{0}^{1}(t) \geq$ $y_{1}^{1}(t)$. Iterando, obtemos $y_{0}^{n}(t) \geq y_{1}^{n}(t)$ e portanto $u_{0}(t) \geq u_{1}(t)$ em $\left[t_{0}, t_{0}+h\right]$.

Seja $t_{+}=\sup \left\{t \in\left[t_{0}, t_{1}\right), u_{0}(t) \geq u_{1}(t)\right\}$. Se $t_{+}$é estritamente menor que o tempo maximal de existência de $u_{0}(t)$ e $u_{1}(t)$, então $t_{+}$é finito e por continuidade, $u_{0}\left(t_{+}\right) \geq$ $u_{1}\left(t_{+}\right)$. Mas então o argumento acima contradiz a definição de $t_{+}$, portanto $u_{0}(t) \geq u_{1}(t)$ enquanto ambas existirem.

ii) Sabemos que $u_{f}\left(t, u_{0}\right)$ e $u_{g}\left(t, u_{0}\right)$ são pontos fixos de $\mathcal{F}_{f}(u)=T\left(t-t_{0}\right) u_{0}+\int_{t_{0}}^{t} T(t-$ $s) f(s, u(s)) d s$ e $\mathcal{F}_{g}(u)=T\left(t-t_{0}\right) u_{0}+\int_{t_{0}}^{t} T(t-s) g(s, u(s)) d s$, respectivamente em $V=$ $\left\{u \in C\left(\left[t_{0}, t_{0}+h\right], X\right),\left\|u(t)-u_{0}\right\|_{X} \leq \delta\right\}$, para $h$ e $\delta$ suficientemente pequenos.

Tomando como função inicial para a iteração $y_{f}^{0}(t)=u_{0}=y_{g}^{0}(t)$, temos por hipótese, para cada $n \geq 0$,

$$
f\left(s, y_{f}^{n}(s)\right) \geq g\left(s, y_{g}^{n}(s)\right)
$$

e portanto, $y_{f}^{n+1}(t)=\mathcal{F}_{f}\left(y_{f}^{n}(t)\right) \geq \mathcal{F}_{g}\left(y_{g}^{n}(t)\right)=y_{g}^{n+1}(t)$ e então $u_{f}\left(t, u_{0}\right) \geq u_{g}\left(t, u_{0}\right)$ em $\left[t_{0}, t_{0}+h\right]$.

Seja $t_{+}=\sup \left\{t \in\left[t_{0}, t_{1}\right), u_{f}\left(t, u_{0}\right) \geq u_{g}\left(t, u_{0}\right)\right\}$. Se $t_{+}$é estritamente menor que o tempo maximal de existência de $u_{f}\left(t, u_{0}\right)$ e $u_{g}\left(t, u_{0}\right)$, então $t_{+}$é finito e por continuidade, $u_{f}\left(t, u_{0}\right)\left(t_{+}\right) \geq u_{g}\left(t_{+}, u_{0}\right)$. Mas então o argumento acima contradiz a definição de $t_{+}$, portanto $u_{f}\left(t, u_{0}\right) \geq u_{g}\left(t, u_{0}\right)$ enquanto elas existirem.

iii) Novamente, $u_{0}(t)=u_{f}\left(t, u_{0}\right)$ e $u_{1}(t)=u_{g}\left(t, u_{1}\right)$ são pontos fixos de

$$
\mathcal{F}_{f}(u)=T\left(t-t_{0}\right) u_{0}+\int_{t_{0}}^{t} T(t-s) f(s, u(s)) d s
$$

e

$$
\mathcal{F}_{g}(u)=T\left(t-t_{0}\right) u_{1}+\int_{t_{0}}^{t} T(t-s) g(s, u(s)) d s,
$$

respectivamente, em $V_{i}=\left\{u \in C\left(\left[t_{0}, t_{0}+h\right], X\right),\left\|u(t)-u_{i}\right\|_{X} \leq \delta\right\}$, para $h$ e $\delta$ suficientemente pequenos.

Começando as iterações com $y_{i}^{0}(t)=u_{i}$, obtemos para cada $n \geq 0$,

$$
f\left(s, y_{0}^{n}(s)\right) \geq g\left(s, y_{1}^{n}(s)\right)
$$


e portanto, $y_{0}^{n+1}(t)=\mathcal{F}_{f}\left(y_{0}^{n}(t)\right) \geq \mathcal{F}_{g}\left(y_{1}^{n}(t)\right)=y_{1}^{n+1}(t)$ e então $u_{0}(t) \geq u_{1}(t)$ em $\left[t_{0}, t_{0}+h\right]$.

Como antes, obtemos que $u_{0}(t) \geq u_{1}(t)$ enquanto existirem. $\square$

\section{Teorema 2.4.4.}

i) Assuma que para todo $r>0$ existe uma constante $\beta=\beta(r)>0$ tal que, para todo $t \in\left[t_{0}, t_{1}\right), f(t, \cdot)+\beta I$ é crescente na bola de raio $r$ de $X$. Então, $u_{0} \geq u_{1}$ implica $u_{f}\left(t, u_{0}\right) \geq u_{f}\left(t, u_{1}\right)$ enquanto ambas existirem.

ii) Assuma que para todo $r>0$ existe constante $\beta=\beta(r)>0$ tal que para $x \geq y e$ $t \in\left[t_{0}, t_{1}\right), f(t, x)+\beta x \geq g(t, y)+y$ na bola de raio $r$ de $X$.

Então, $u_{0} \geq u_{1}$ implica $u_{f}\left(t, u_{0}\right) \geq u_{g}\left(t, u_{1}\right)$ enquanto ambas existirem.

\subsubsection{O caso parabólico}

Nesta seção tratamos da comparação para problemas semilineares da forma (2.2) onde $-A$ é um operador setorial em $X, f:\left[t_{0}, t_{1}\right) \times X^{\alpha} \rightarrow X^{\beta}$ é localmente Hölder contínua em $t$, localmente Lipschitz contínua em $u$ e $0 \leq \alpha-\beta<1$. A solução de (2.2) e denotada por $u_{f}\left(t, u_{0}\right)$. Não demonstraremos os resultados a seguir, pois eles são análogos dos teoremas da Seção 2.4.1.

Se $(X, \leq)$ é um espaço de Banach ordenado, o resultado a seguir estabelece condições sobre $f$ que asseguram a invariância do cone positivo $C$ de $X$.

Teorema 2.4.5. Seja $(X, \leq)$ um espaço de Banach ordenado, e seja $\{T(t): t \geq 0\} \subset$ $L(X)$ o semigrupo analítico de operadores lineares positivos gerado pelo operador $A$ onde - A é setorial. Assuma que para todo $t \in\left[t_{0}, t_{1}\right), f(t, \cdot)$ é positivo. Então, $u_{0} \geq 0$ implica $u_{f}\left(t, u_{0}\right) \geq 0$, enquanto existir.

Teorema 2.4.6. Com as notações acima assuma que para todo $r>0$ existe uma constante $\beta=\beta(r)>0$ tal que para todo $t \in\left[t_{0}, t_{1}\right), f(t, \cdot)+\beta I$ é positivo na bola de raio $r$ em $X^{\alpha}$.

Então, se $u_{0} \geq 0, u_{f}\left(t, u_{0}\right)$ é positiva enquanto existir.

Relativamente a comparação, temos o seguinte resultado: 
Teorema 2.4.7. Süponha que $(X, \leq)$ é um espaço de Banach ordenado e $-A$ um operador setorial tal que o semigrupo analítico gerado por $A$ é positivo.

i) Assuma que para todo $t \in\left[t_{0}, t_{1}\right), f(t, \cdot)$ é crescente. Então $u_{0} \geq u_{1}$ implica $u_{f}\left(t, u_{0}\right) \geq$ $u_{f}\left(t, u_{1}\right)$ enquanto ambas existirem.

ii) Assuma que $f, g$ verificam $f(t, x) \geq g(t, x)$ para todo $t$ e $x$. Então, $u_{f}\left(t, u_{0}\right) \geq u_{g}\left(t, u_{0}\right)$ enquanto ambas existirem.

iii) Assuma $f, g$ são tais que $x \geq y$ implica $f(t, x) \geq g(t, y)$. Então, $u_{0} \geq u_{1}$ implica $u_{f}\left(t, u_{0}\right) \geq u_{g}\left(t, u_{1}\right)$ enquanto ambas existirem.

\section{Teorema 2.4.8.}

i) Assuma que para todo $r>0$ existe uma constante $\beta=\beta(r)>0$ tal que para todo $t \in\left[t_{0}, t_{1}\right), f(t, \cdot)+\beta I$ é crescente na bola de raio $r$ de $X^{\alpha}$. Então, $u_{0} \geq u_{1}$ implica $u_{f}\left(t, u_{0}\right) \geq u_{f}\left(t, u_{1}\right)$ enquanto ambas existirem.

ii) Assuma que para todo $r>0$ existe $\beta=\beta(r)>0$ tal que para $t \in\left[t_{0}, t_{1}\right)$, ex $x y$ $f(t, x)+\beta x \geq g(t, y)+\beta y$ na bola de raio $r$ de $X^{\alpha}$. Então, $u_{0} \geq u_{1}$ implica $u_{f}\left(t, u_{0}\right) \geq$ $u_{g}\left(t, u_{1}\right)$ enquanto ambas existirem.

\subsection{Sub-soluções e Super-soluções}

Seja $(X, \leq)$ um espaço de Banach ordenado e $\{T(t): t \geq 0\} \subset L(X)$ um $C_{0}$-semigrupo de operadores lineares positivos. Sob essas hipóteses temos:

Definição 2.5.1. A funcão $v \in C\left(\left[t_{0}, t_{1}\right], X\right)$ é a subsolução de (2.2) se e somente se

$$
v_{t} \leq A v+f(t, v)
$$

no sentido que para todo $t_{0} \leq r \leq t \leq t_{1}$ temos

$$
v(t) \leq T(t-r) v(r)+\int_{r}^{t} T(t-s) f(s, v(s)) d s
$$

Analogamente, definimos uma supersolução simplesmente invertendo a desigualdade. 
Note que se $v(t)$ é uma solução forte de $v_{t}=A v+g(t)$ e $g(t) \leq f(t, v)$ então, usando a fórmula da variação das constantes e a positividade do semigrupo e do operador integral, obtemos que $v(t)$ verifica a desigualdade acima. De fato:

$$
v(t)=T(t-r) v(r)+\int_{r}^{t} T(t-s) g(s) d s \leq T(t-r) v(r)+\int_{r}^{t} T(t-s) f(s, v(s)) d s
$$

Teorema 2.5.1. Assuma que para todo $t \in\left[t_{0}, t_{1}\right), f(t, \cdot)$ é crescente.

Seja $v(t)$ uma subsolução de (2.2). Então $v(t) \leq u_{f}\left(t, v\left(t_{0}\right)\right)$ enquanto ambas existirem. Adicionalmente, se $u_{0}$ é tal que $v\left(t_{0}\right) \leq u_{0}$, então $v(t) \leq u_{f}\left(t, u_{0}\right)$ enquanto ambas existirem.

O mesmo vale para supersoluções, com as desigualdades invertidas.

Como uma conseqüência imediata, obtemos

Corolário 2.5.1. Assuma que $v(t)$ e $V(t)$ são sub e supersoluções de (2.2), respectivamente e tais que $v(t) \leq V(t)$ para todo $t$ e assuma que para todo $r>0$ existe uma constante $\beta=\beta(r)>0$ tal que para todo $t \in\left[t_{0}, t_{1}\right), f(t, \cdot)+\beta I$ é crescente na bola de raio $r$ em $X$.

Então $v\left(t_{0}\right) \leq u_{0} \leq V\left(t_{0}\right)$, temos

$$
v(t) \leq u_{f}\left(t, u_{0}\right) \leq V(t)
$$

enquanto ambas existirem. 


\section{Capítulo 3}

\section{Aplicações}

\subsection{A Equação do Calor Semilinear}

Considere o seguinte problema de valor inicial e de fronteira

$$
\begin{aligned}
& u_{t}(t, x)=u_{x x}(t, x)-u(t, x)+f(u)(t, x), \quad 0<x<1, t>0 \\
& u(0, x)=u(1, x)=0, \\
& u(0, x)=u_{0}(x) \in H_{0}^{1} .
\end{aligned}
$$

onde $f: \mathbb{R} \rightarrow \mathbb{R}$ é diferenciável com derivada localmente limitada.

Teorema 3.1.1. Seja o operador

$$
A: H^{2} \cap H_{0}^{1} \subset L^{2} \rightarrow L^{2}
$$

tal que

$$
A u=-u_{x x}+u \in L^{2}
$$

Temos que:

(i) $A^{\frac{1}{2}}$ é auto-adjunta.

(ii) A tem resolvente positivo. 
Prova: (i) Note que:

$$
\begin{gathered}
A u, u\rangle_{L^{2}}=\left\langle-u^{\prime \prime}+u, u\right\rangle_{L^{2}}=\int\left(-u^{\prime \prime}+u\right) u d x= \\
=\int-u^{\prime \prime} u d x+\int u^{2} d x=\int\left(u^{\prime}\right)^{2} d x+\int u^{2} d x=\left\|u^{\prime}\right\|_{L^{2}}^{2}+\|u\|_{L^{2}}^{2}=\|u\|_{H^{1}}^{2}>0
\end{gathered}
$$

e

$$
\begin{gathered}
\langle A u, v\rangle_{L^{2}}=\left\langle-u^{\prime \prime}+u, v\right\rangle_{L^{2}}=\int\left(-u^{\prime \prime}+u\right) v d x= \\
=\int-u^{\prime \prime} v d x+\int u v d x=\int u^{\prime} v^{\prime} d x+\int u v d x=\langle u, A v\rangle
\end{gathered}
$$

sempre que $u, v \in D(A)$.

Com isso, concluímos que $A$ é simétrico. Ainda do Teorema 1.10.1 (iii), sabemos que se $f \in L^{2}$ e $u \in H_{0}^{1}$ é solução fraca de $-u^{\prime \prime}+u=f$ então $u \in H^{2}$ e, portanto $A$ é sobrejetora. Logo $A$ é auto-adjunto. E assim, temos que $A^{\frac{1}{2}}$ também o é.

ii) Como $A^{\frac{1}{2}}$ é auto-adjunto, então

$$
\left\langle A^{\frac{1}{2}} A^{\frac{1}{2}} u, u\right\rangle=\left\langle A^{\frac{1}{2}} u, A^{\frac{1}{2}} u\right\rangle=\left\|u^{\prime}\right\|_{L^{2}}^{2}+\|u\|_{L^{2}}^{2}, \quad \forall u \in D(A)
$$

portanto,

$$
\|u\|_{X^{\frac{1}{2}}}=\|u\|_{H^{1}}, \quad \forall u \in D(A)
$$

e

$$
\overline{D(A)}^{H^{1}}=\overline{D(A)}^{X^{\frac{1}{2}}}=X^{\frac{1}{2}}
$$

Provaremos agora que $A$ tem resolvente positivo, isto é,

$$
\left\langle A^{\frac{1}{2}} u, A^{\frac{1}{2}} u\right\rangle=\left\langle A^{\frac{1}{2}}|u|, A^{\frac{1}{2}}|u|\right\rangle
$$

Para isso, dado $u \in H_{0}^{1}$, defina $|u|$ como segue

$$
|u|(x)=|u(x)|
$$


Temos que $|u| \in H_{0}^{1} \mathrm{e}$

$$
\begin{aligned}
\left\langle A^{\frac{1}{2}} u, A^{\frac{1}{2}} u\right\rangle & =\int\left(u^{\prime}(x)\right)^{2} d x+\int(u(x))^{2} d x \\
& =\int\left|u^{\prime}(x)\right|^{2} d x+\int|u(x)|^{2} d x \\
& =\int\left(|u|^{\prime}(x)\right)^{2} d x+\int(|u|(x))^{2} d x \\
& =\left\||u|^{\prime}\right\|_{L^{2}}^{2}+\||u|\|_{L^{2}}^{2} \\
& =\left\langle A^{\frac{1}{2}}|u|, A^{\frac{1}{2}}|u|\right\rangle
\end{aligned}
$$

Com isso, segue o resultado. $\square$

Defina a seguinte função $f^{e}: H_{0}^{1} \rightarrow L^{2}$, tal que $\left(f^{e}(u)\right)(x)=f(u(x))$. Mostremos que $f^{e}$ está bem definida. De fato, se $u \in H_{0}^{1}$ temos pela observaçāo 1.9.1 que $u \in C(\bar{I})$. Dessa forma,

$$
\sup _{x \in I}|f(u(x))| \leq k<\infty .
$$

Com isso, concluímos que $f^{e}(u) \in L^{2}$, pois:

$$
\int\left(f^{e}(u)(x)\right)^{2}=\int(f(u(x)))^{2} \leq \int K^{2}<\infty .
$$

Logo $f^{e}$ está bem definida.

Teorema 3.1.2. $f^{e}$ é Lipschitz em subconjuntos limitados de $H_{0}^{1}$.

Prova: Seja $B=\left\{u \in H_{0}^{1}:\|u\|_{H_{0}^{1}} \leq M\right\}$ e $u, \tilde{u} \in B$. Temos que $u, \tilde{u} \in C(\bar{I})$ e, portanto $\|u\|_{\infty},\|\bar{u}\|_{\infty} \leq M$.

E ainda,

$$
\left|f^{e}(u)(x)-f^{e}(\tilde{u})(x)\right|=|f(u(x))-f(\tilde{u}(x))| \leq L(B)|u(x)-\tilde{u}(x)|
$$

o que implica,

$$
\left\|f^{e}(u)-f^{e}(\tilde{u})\right\|_{L^{2}} \leq \tilde{L}(B)\|u-\bar{u}\|_{H_{0}^{1} \cdot \square}
$$


Como $u:\left[0, \tau_{u_{0}}\right] \rightarrow H_{0}^{1}$ é uma solução de 3.1 então $u$ é uma curva em $H_{0}^{1}$. E ainda $\left\{\left(t, u_{0}\right): t \in I_{u_{0}}=\left[0, \tau_{u_{0}}\right]\right\}$ é um compacto, logo $\left\{u\left(t, u_{0}\right): t \in I_{u_{0}}=\left[0, \tau_{u_{0}}\right]\right\}$ e, portanto $\left\{u\left(t, u_{0}\right): t \in I_{u_{0}}=\left[0, \tau_{u_{0}}\right]\right\}$ é limitada.

Analogamente para $u_{1}$ tal que $u_{0} \geq u_{1}$.

Assim, temos:

$$
\sup _{t \in I_{u_{0}}}\left\|u\left(t, u_{0}\right)\right\|_{\infty} \leq K
$$

$\mathrm{e}$

$$
\sup _{t \in I_{u_{1}}}\left\|u\left(t, u_{1}\right)\right\|_{\infty} \leq K
$$

O nosso objetivo agora é deixar $f$ crescente, para que possamos aplicar o Teorema 2.4.3; nas considerações acima.

Como $f: \mathbb{R} \rightarrow \mathbb{R}$ é diferenciável com derivada localmente limitada, então se $s, s+h \in$ $[-K, K]$, temos $|f(s+h)-f(s)| \leq M_{s}|h|$. Donde, segue que $\sup _{|s| \leq K}\left|f^{\prime}(s)\right|=\beta$.

Logo

$$
\beta+f^{\prime}(s) \geq 0, \quad \forall s \in[-K, K]
$$

Tomando $\tilde{f}(s)=f(s)+\beta s, \quad \forall s \in[-K, K]$.

Por (4.3), temos $\tilde{f}^{\prime}(s)=f^{\prime}(s)+\beta \geq 0, \quad \forall s \in[-K, K]$, o que implica que $\tilde{f}$ é crescente em $I=[-K, K]$.

Se $s \in(-\infty,-K]$, tomamos $\tilde{f}(s)=a s+b$, onde $a=f^{\prime}(-K)+\beta$ e $a(-K)+b=\tilde{f}(-K)$. Se $s \in[K, \infty)$, tomamos $\tilde{f}(s)=a s+b$, onde $a=f^{\prime}(K)+\beta$ e $a(K)+b=\tilde{f}(K)$.

Portanto $\tilde{f}$ assim definida é crescente então $\tilde{f}^{e}$ é crescente.

Pelo Teorema 2.4.3, temos que se $u_{0} \geq u_{1}$ então

$$
u\left(t, u_{0}, \tilde{f}^{e}-\beta\right) \geq u\left(t, u_{1}, \tilde{f}^{e}-\beta\right)
$$

enquanto as soluções existirem.

Teorema 3.1.3. Se $u_{0} \geq u_{1}$ então

$$
u\left(t, u_{0}, f^{e}\right)=u\left(t, u_{0}, \tilde{f}^{e}-\beta\right) \geq u\left(t, u_{1}, \tilde{f}^{e}-\beta\right)=u\left(t, u_{1}, f^{e}\right), \quad \forall t \in I_{u_{0}} \cap I_{u_{1}} .
$$

Analogamente, temos: 
Teorema 3.1.4. Se $f_{0}^{e} \geq f_{1}^{e}$ então

$$
u\left(t, u_{0}, f_{0}^{e}\right) \geq u\left(t, u_{0}, f_{1}^{e}\right)
$$

enquanto ambas existirem.

\subsection{Um Exemplo em Dimensão Finita}

Como uma segunda aplicação dos resultados da seção sobre "Critérios de Comparação Abstratos," mostramos que para um espaço $H$ de dimensão finita e um operador $A$ dado por uma matriz a ser especificada, as hipóteses do Teorema 2.3.1 estão satisfeitas.

Seja a matriz $n \times n$.

$$
\left(\begin{array}{ccccc}
1 & -1 & 0 & \cdots & 0 \\
-1 & 2 & -1 & \ddots & \vdots \\
0 & -1 & 2 & \ddots & 0 \\
\vdots & \ddots & \ddots & \ddots & -1 \\
0 & \cdots & 0 & -1 & 1
\end{array}\right)
$$

Ela é chamada matriz do tipo Jacobi.

Mostremos inicialmente que:

$$
\langle A u, u), \quad \forall u=\left(u_{1}, u_{2}, \ldots, u_{n}\right) \in \mathbb{R}^{n} .
$$

De fato:

$$
\begin{gathered}
\langle A u, u\rangle=\left(u_{1}-u_{2}\right) u_{1}+\left(-u_{1}+2 u_{2}-u_{3}\right) u_{2}+\left(-u_{2}+2 u_{3}-u_{4}\right) u_{3} \\
+\left(-u_{j-2}+2 u_{j-1}-u_{j}\right) u_{j-1}+\left(-u_{j-1}+2 u_{j}-u_{j+1}\right) u_{j}+\ldots \\
+\left(-u_{n-2}+2 u_{n-1}-u_{n}\right) u_{n-1}+\left(-u_{n-1}+u_{n}\right) u_{n}
\end{gathered}
$$

Portanto

$$
\langle A u, u\rangle=\left(u_{1}-u_{2}\right)^{2}+\left(u_{2}-u_{3}\right)^{2}+\ldots+\left(u_{j-1}-u_{j}\right)^{2}+\ldots+\left(u_{n-1}-u_{n}\right)^{2} \geq 0 .
$$


E assim, segue que:

$$
\langle A u, u\rangle \geq 0, \quad \forall u \in \mathbb{R}^{n}
$$

Então $A: \mathbb{R}^{n} \rightarrow \mathbb{R}^{n}$ é um operador auto-adjunto e positivo. Temos que:

1) $(A+\alpha)^{-1} \mathbb{R}^{n} \subset \mathbb{R}^{n}$;

2) Para cada $u=\left(u_{1}, u_{2}, \ldots, u_{n}\right) \in \mathbb{R}^{n}$ podemos definir $|u|=\left(\left|u_{1}\right|,\left|u_{2}\right|, \ldots,\left|u_{n}\right|\right) \in \mathbb{R}^{n} \cap C$ e esta relação satisfaz: Um elemento $u \in \mathbb{R}^{n}$ está em $C$ se e somente se $u=|u|$ e $\|u\|=\||u|\|$, pois $\|u\|=\sqrt{u_{1}^{2}+u_{2}^{2}+\ldots+u_{n}^{2}}$;

3) Dados $v=\left(v_{1}, v_{2}, \ldots, v_{n}\right) \in C$ e $u \in \mathbb{R}^{n}$, temos:

$$
\left|u_{1}\right| v_{1}+\left|u_{2}\right| v_{2}+\ldots+\left|u_{n}\right| v_{n}=\left|u_{1}\right|\left|v_{1}\right|+\left|u_{2}\right|\left|v_{2}\right|+\ldots+\left|u_{n}\right|\left|v_{n}\right| \geq\left|u_{1} v_{1}+u_{2} v_{2}+\ldots+u_{n} v_{n}\right|
$$

Portanto:

$$
\langle|u|, v\rangle \geq|\langle u, v\rangle|, \quad \forall u \in \mathbb{R}^{n}, \forall v \in C .
$$

E ainda se $u \in D\left(A^{\frac{1}{2}}\right)$ então $|u| \in D\left(A^{\frac{1}{2}}\right\rangle \mathrm{e}$

$$
\begin{gathered}
(A u, u\rangle \geq\langle A|u|,|u|\rangle \Rightarrow \\
\left(A^{\frac{1}{2}} A^{\frac{1}{2}} u, u\right\rangle \geq\left\langle A^{\frac{1}{2}} A^{\frac{1}{2}}|u|,|u|\right\rangle \Rightarrow \\
\left\langle A^{\frac{1}{2}}|u|, A^{\frac{1}{2}}|u|\right\rangle \leq\left(A^{\frac{1}{2}} u, A^{\frac{1}{2}} u\right\rangle .
\end{gathered}
$$

Com todas as consideraçōes acima, temos pelo Teorema 2.3.1, segue que $(A+\alpha\rangle^{-1}$ é crescente para todo $\alpha>0$.

Como $A \in L\left(\mathbb{R}^{n}\right)$, então $A$ é o gerador do semigrupo $T(t)=\mathrm{e}^{t A}$. Sabemos que $T(t) x_{0}=\mathrm{e}^{t A} x_{0}$ é solução da equação diferencial:

$$
\left\{\begin{array}{l}
\frac{d x}{d t}(t)=A x(t) \\
x(0)=x_{0} \geq 0
\end{array}\right.
$$

Como $(A+\alpha)^{-1}, \quad \forall \alpha>0$ é crescente então pelo Teorema 2.2.1, T(t)= $\mathrm{e}^{t A}$ é crescente para todo $t \geq 0$. Logo como $x_{0} \geq 0$, então $\mathrm{e}^{t A} x_{0} \geq 0, \quad \forall t \geq 0$.

Com isso, acabamos de provar o seguinte teorema:

Teorema 3.2.1. Se $x_{0} \geq x_{1}$ então $T(t) x_{0} \geq T(t) x_{1}$, para todo $t \geq 0$.

Analogamente, temos: 
Teorema 3.2.2. Considere os problemas:

$$
\left\{\begin{array}{l}
\frac{d x}{d t}(t)=A x(t)+f_{i}(x) \\
x(0)=x_{i} \geq 0 i=0,1 .
\end{array}\right.
$$

Então se $x_{0} \geq x_{1}$ temos que $x\left(t, x_{0}, f_{0}\right) \geq x\left(t, x_{1}, f_{1}\right)$, enquanto as soluções existirem. 


\section{Referências Bibliográficas}

[1] H. Amann, Linear and Quasilinear Parabolic Problems. Abstract Linear Theory. Birkhäuser Verlag, 1995.

[2] J. Arrieta \& A. N. Carvalho, Abstract Parabolic Problems with Critical Nonlinearities and Applications to Navier-Stokes and Heat Equations, Notas do ICMSC-USP, Série Matematica \# 51, (1997).

[3] H. Brézis, Analyse Fonctionnelle, Masson Editeur, París, (1983).

[4] N. Dunford and J. Schwartz, Linear Operators-Part I, John Wiley \& Sons, 1988.

[5] M. A. Evgrafov, Asymptotic Estimates and Entire Functions, Gordon and Breach, New York (1961).

[6] D. Henry, Geometric theory of semilinear parabolic equations, Lecture Notes in Mathematics 840, Springer-Verlag, Berlin, (1981).

[7] D. Henry, Semigroups, Notas manuscritas, IME-USP, Brazil (1981).

[8] A. Pazy, Semigroups of Linear Operators and Applications to Partial Differential Equations, Applied Math. Sciences 44, Springer Verlag, New York (1983). 